Supporting Information

\title{
KPF6-Mediated Esterification and Amidation of Carboxylic Acids
}

Sonam, ${ }^{\text {a }}$ Vikki N. Shinde ${ }^{\mathrm{a}}$ and Anil Kumar*,a

Department of Chemistry, Birla Institute of Technology \& Science Pilani, Pilani Campus, Rajasthan, 333031, India.

E-mail: anilkumar@pilani.bits-pilani.ac.in

Table of contents:

\begin{tabular}{|c|l|l|}
\hline 1. & Copies of NMR $\left({ }^{1} \mathrm{H}\right.$ and $\left.{ }^{13} \mathrm{C}\right)$ for esters and amides (3aa-vd \& 5aa-ld) & S1-S48 \\
\hline 2. & Copy of HMRS of $\mathbf{1 t}{ }^{-18} \mathbf{O}$ and intermediate & S49 \\
\hline 3. & Green metrics calculations & S49-S50 \\
\hline 4 & References & S50 \\
\hline
\end{tabular}

1. Copies of NMR ( ${ }^{1} \mathrm{H}$ and ${ }^{13} \mathrm{C}$ ) for esters and amides (3aa-vd \& 5aa-ld)

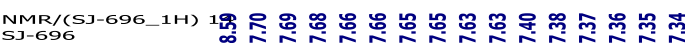

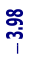

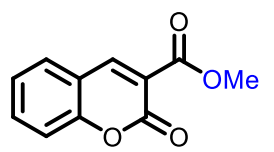

3aa, ${ }^{1} \mathrm{H}$ NMR

$\mathrm{CDCl}_{3}, 400 \mathrm{MHz}$

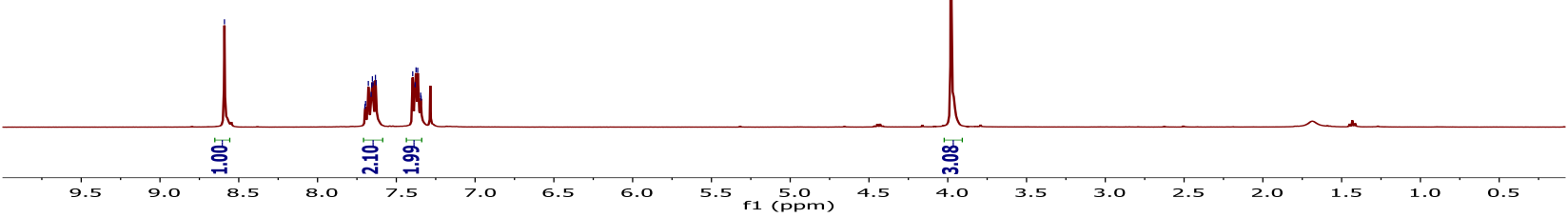

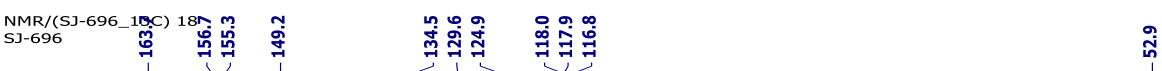

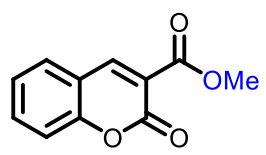

3aa, ${ }^{13} \mathrm{C}\left\{{ }^{1} \mathrm{H}\right\}$ NMR

$\mathrm{CDCl}_{3}, 100 \mathrm{MHz}$

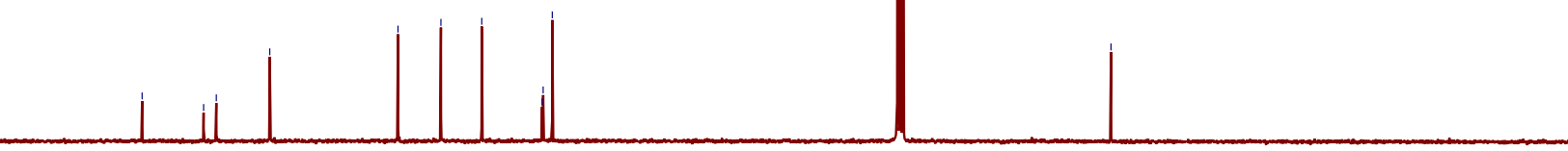




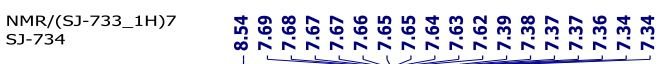

事得早毒

早是
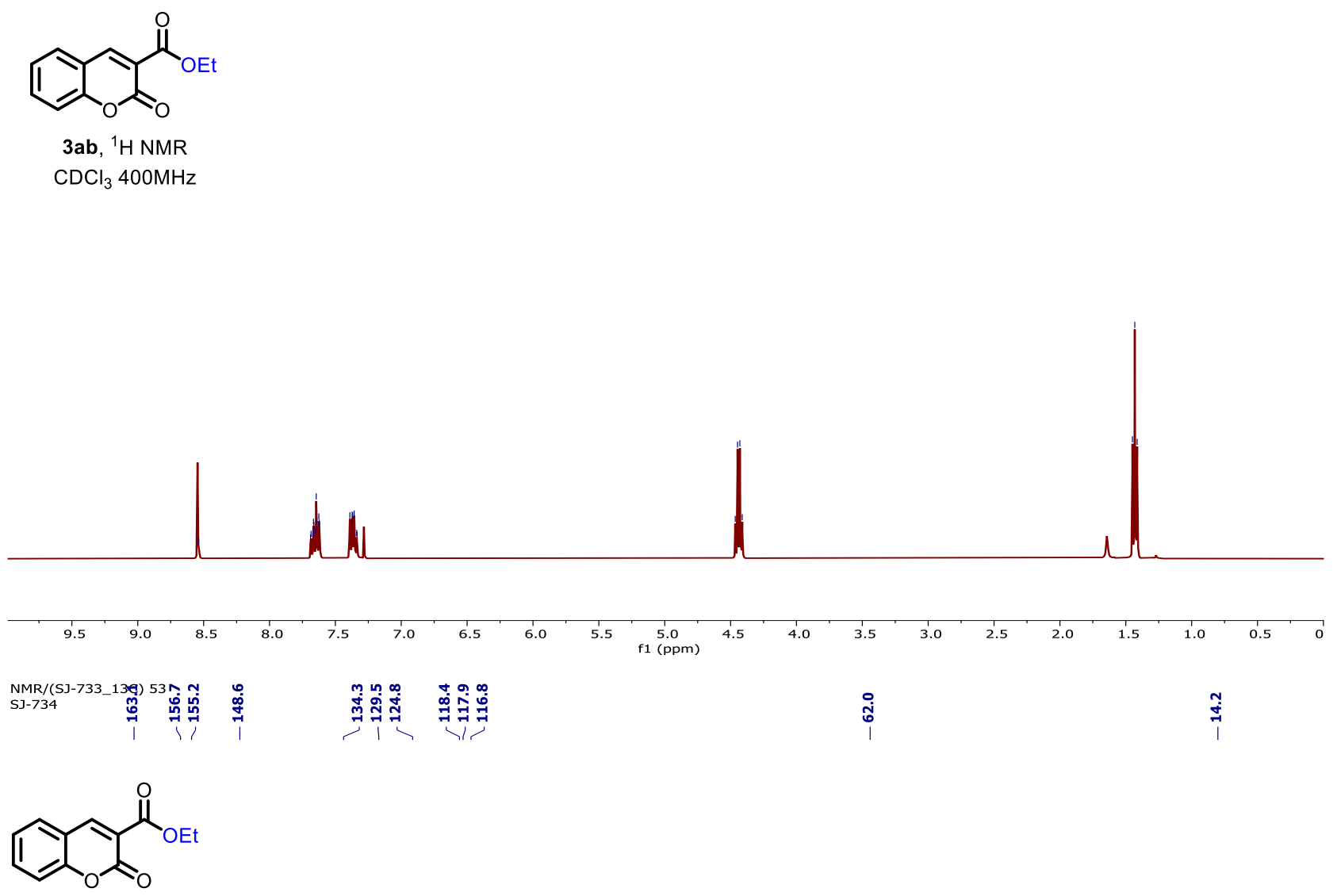

3ab, ${ }^{13} \mathrm{C}\left\{{ }^{1} \mathrm{H}\right\}$ NMR $\mathrm{CDCl}_{3} 100 \mathrm{MHz}$
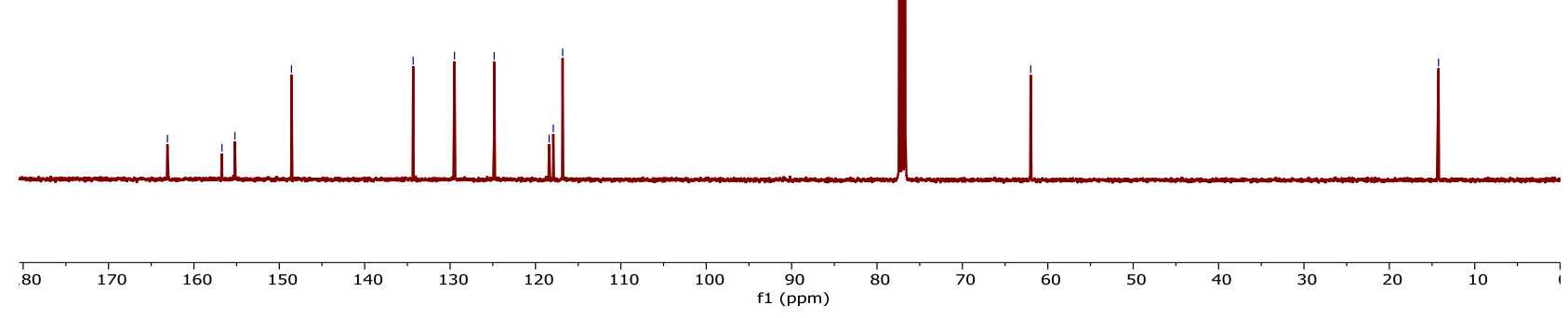

S2 


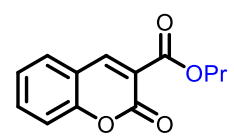

3ac, ${ }^{1} \mathrm{H}$ NMR

$\mathrm{CDCl}_{3} 400 \mathrm{MHz}$

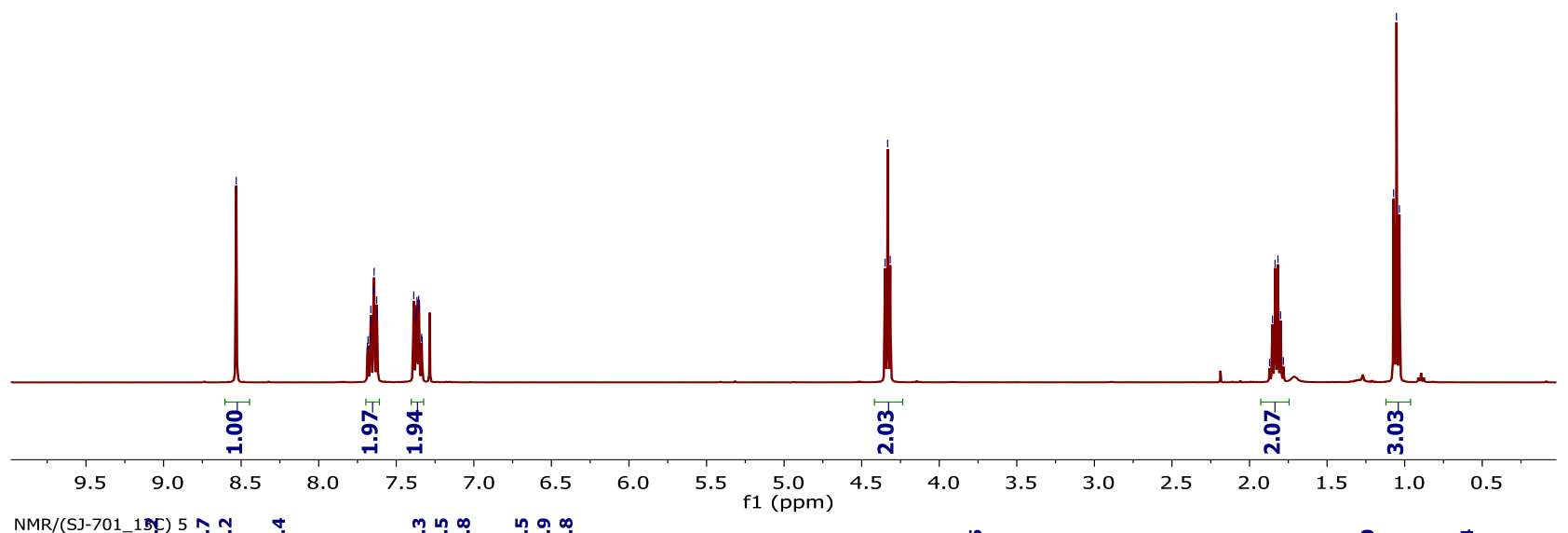

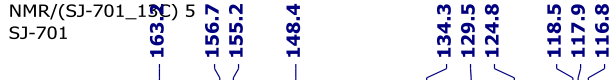

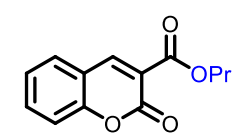

3ac, ${ }^{13} \mathrm{C}\left\{{ }^{1} \mathrm{H}\right\}$ NMR

$\mathrm{CDCl}_{3} 100 \mathrm{MHz}$
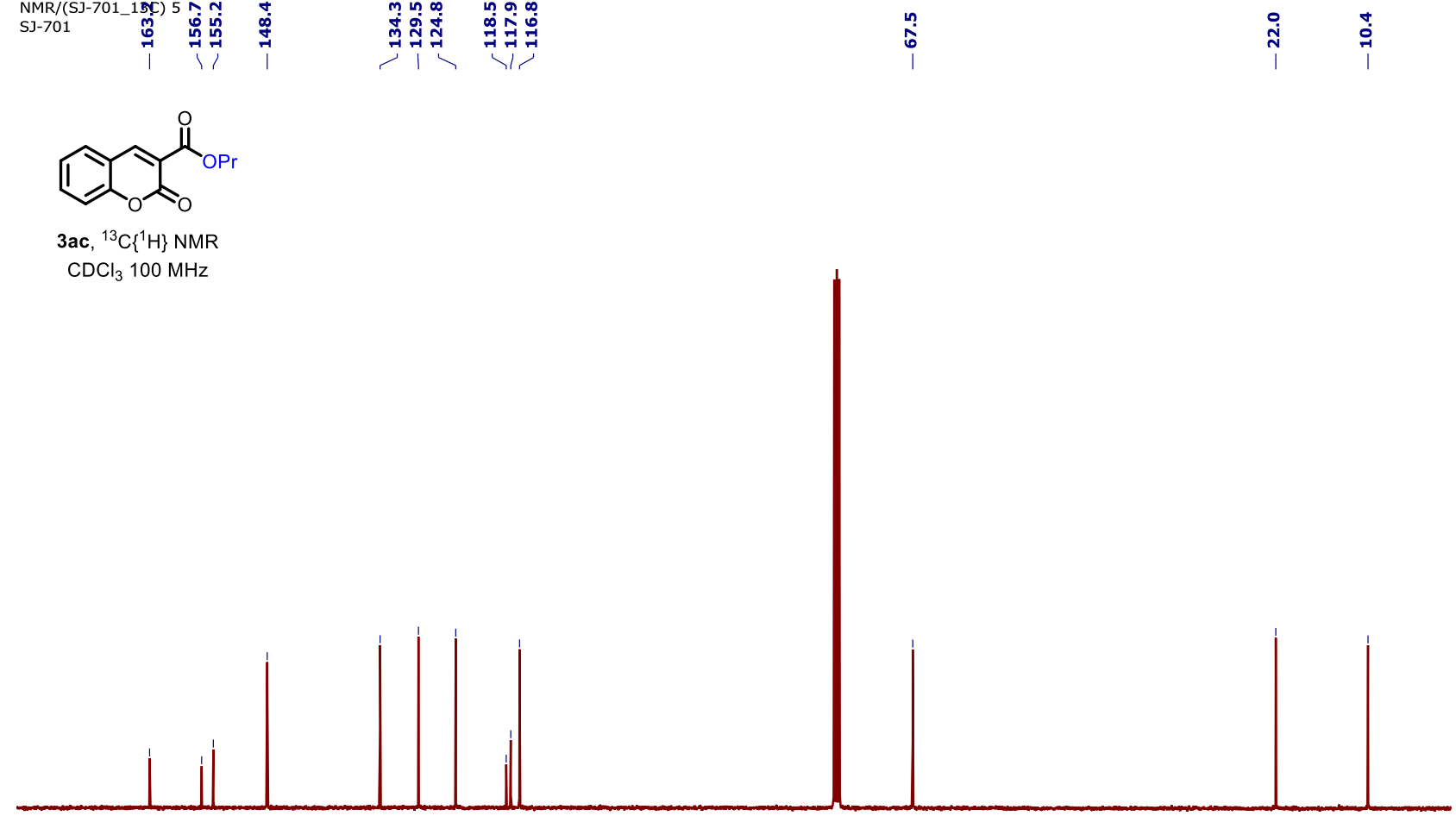

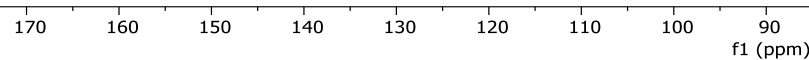

$80 \quad 70 \quad 60 \quad 50$

40

$20 \quad 10$ 


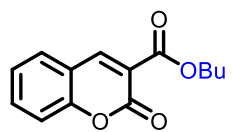

3ad, ${ }^{1} \mathrm{H}$ NMR

$\mathrm{CDCl}_{3} 400 \mathrm{MHz}$

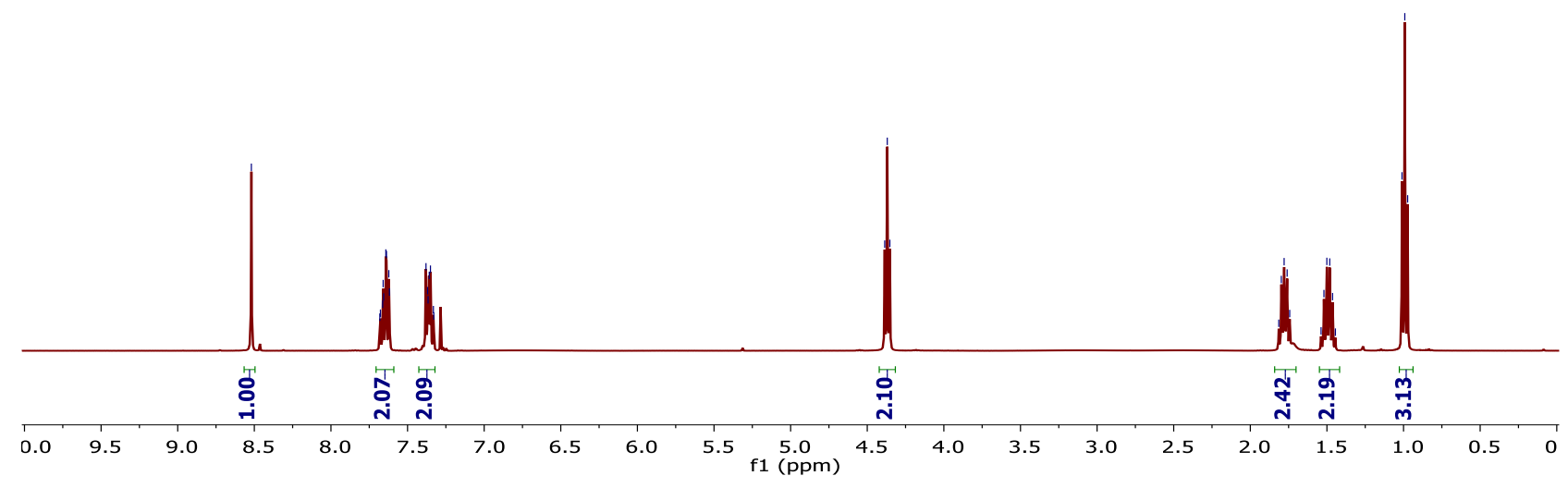

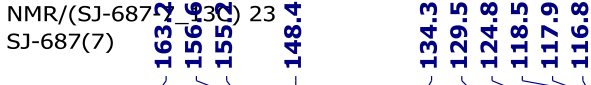

$\stackrel{\infty}{\leftrightarrow: 0}$

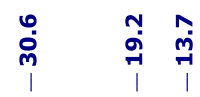

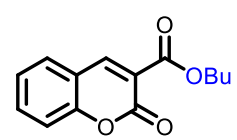

3ad, ${ }^{13} \mathrm{C}\left\{{ }^{1} \mathrm{H}\right\}$ NMR

$\mathrm{CDCl}_{3} 100 \mathrm{MHz}$

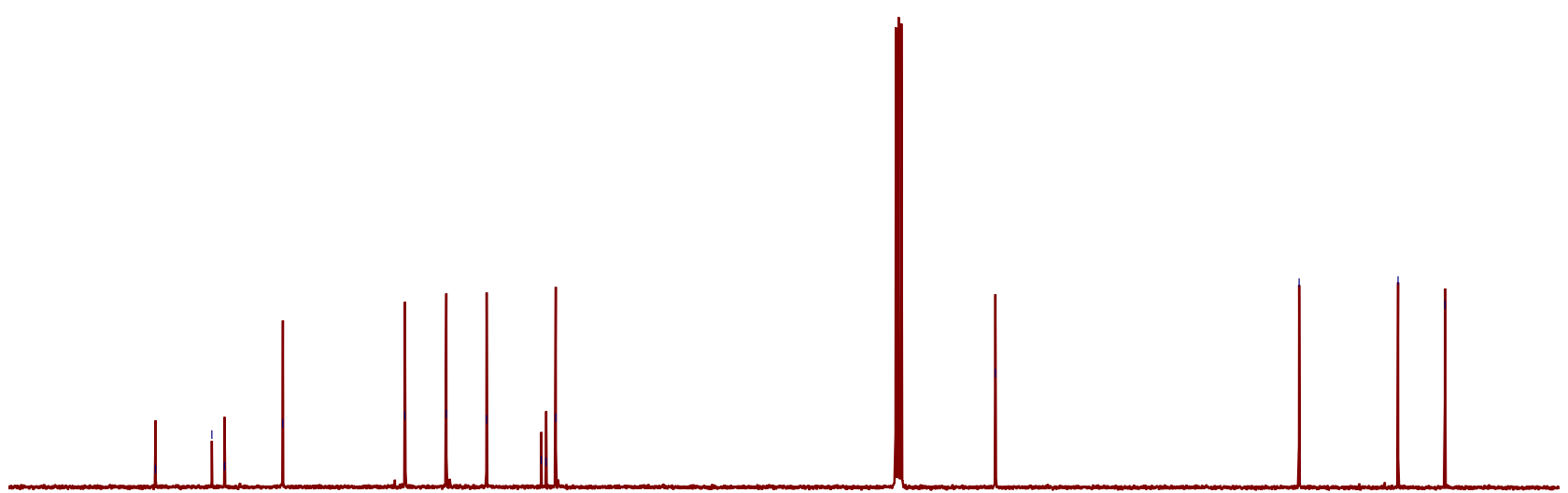

80

$\begin{array}{lllllll}170 & 160 & 150 & 140 & 130 & 120 & 110\end{array}$

$\stackrel{90}{f 1(\mathrm{ppm})} 8$

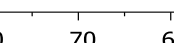

$\begin{array}{lllll}50 & 40 & 30 & 20 & 10\end{array}$ 


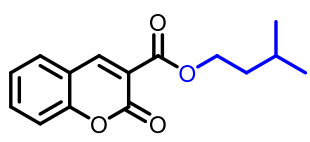

3ae, ${ }^{1} \mathrm{H}$ NMR

$\mathrm{CDCl}_{3} 400 \mathrm{MHz}$

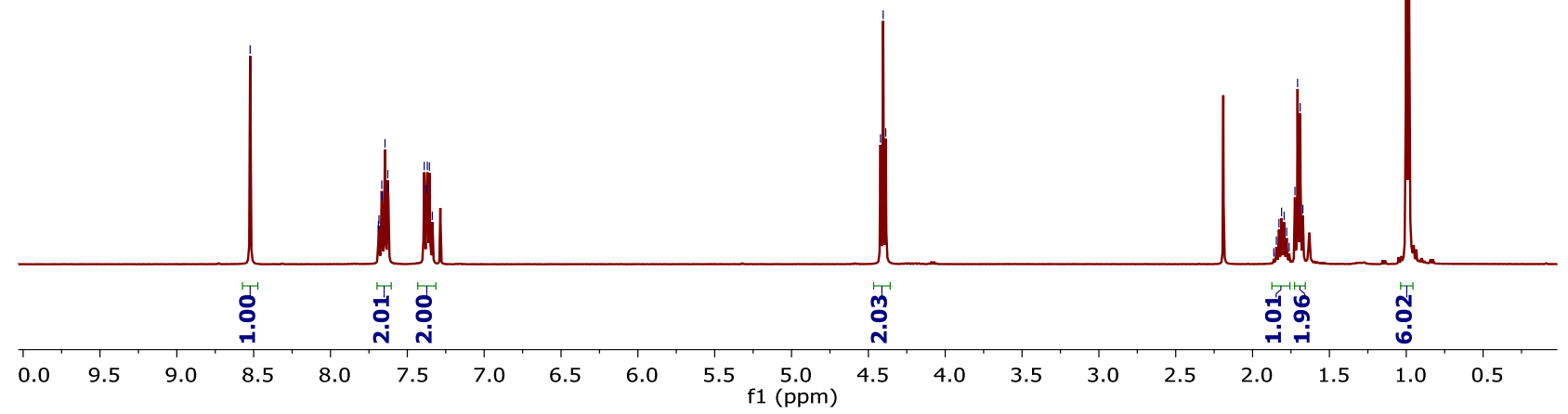

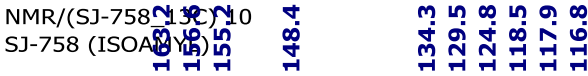

કั่

$\stackrel{m}{m} \quad \stackrel{+}{m} \stackrel{n}{N}$

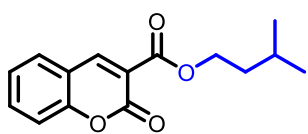

3ae, ${ }^{13} \mathrm{C}\left\{{ }^{1} \mathrm{H}\right\}$ NMR

$\mathrm{CDCl}_{3} 100 \mathrm{MHz}$ . 


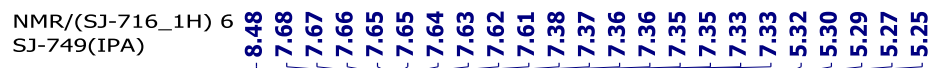
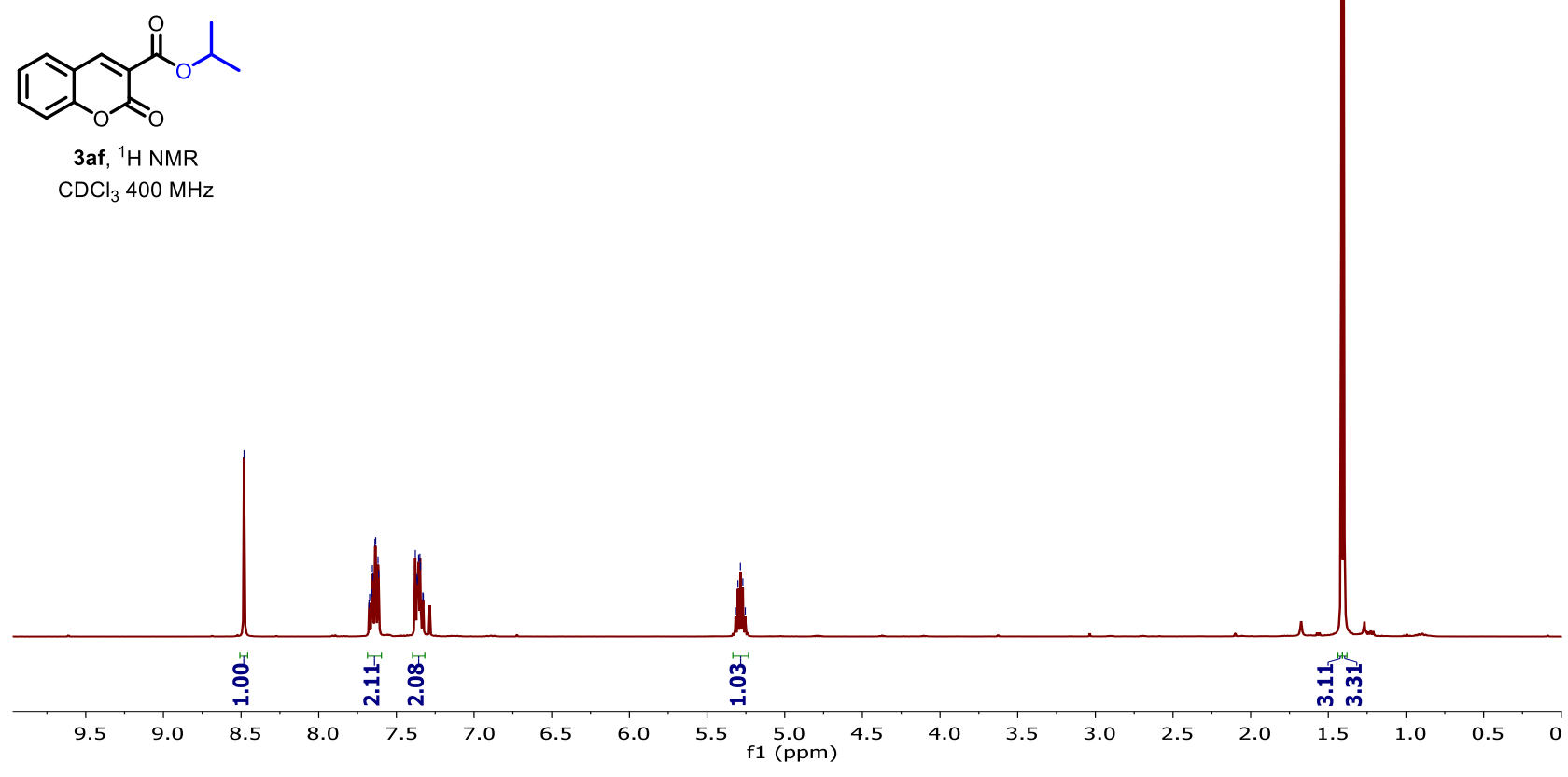

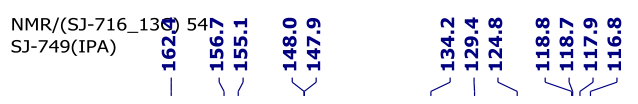

sì

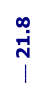
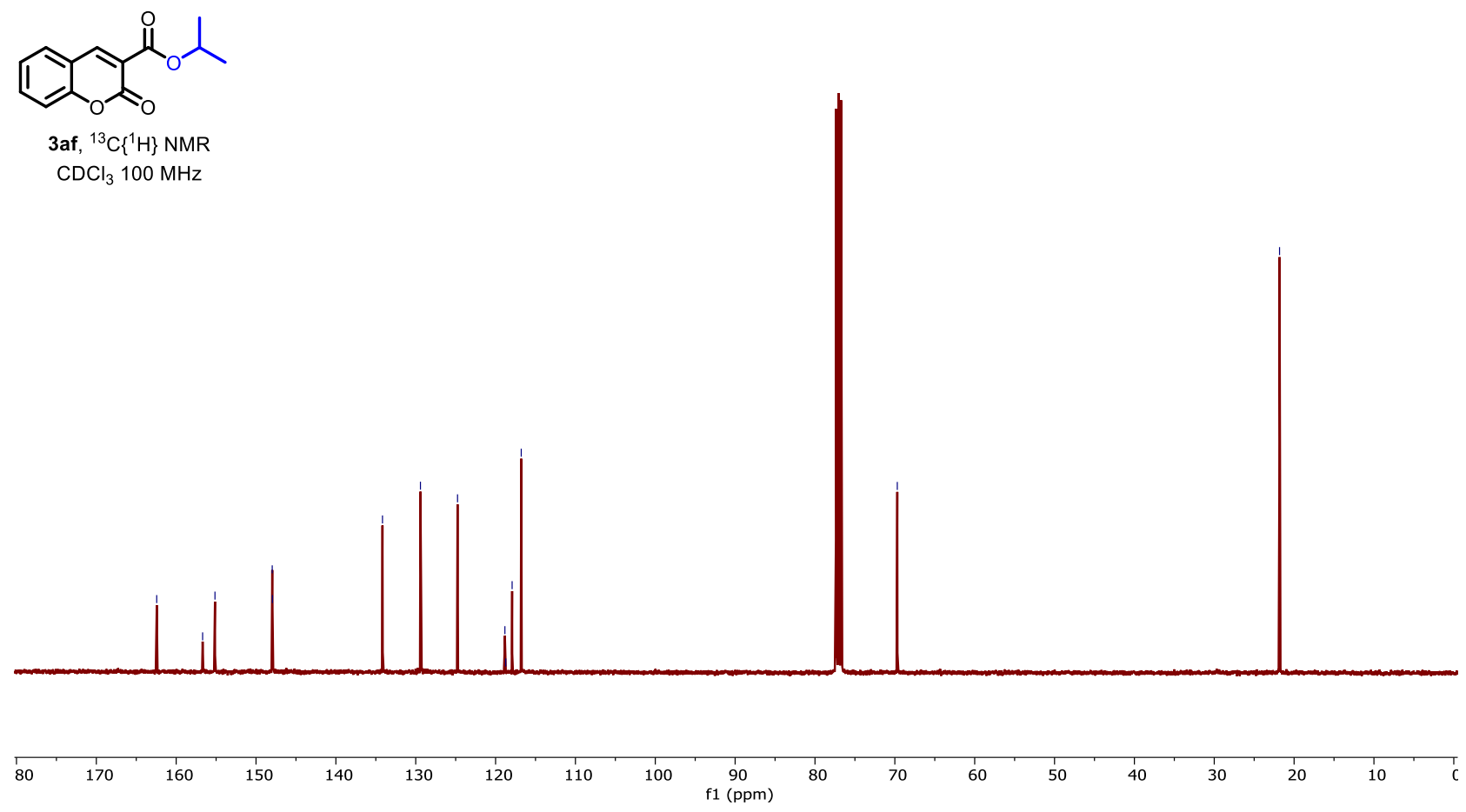

S6 


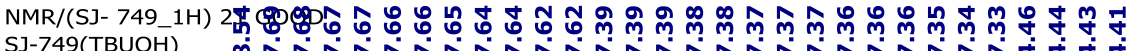

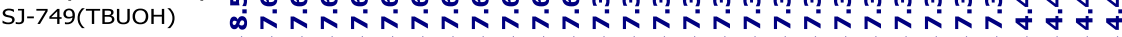

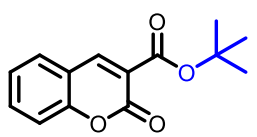

3ag, ${ }^{1} \mathrm{H}$ NMR

$\mathrm{CDCl}_{3} 400 \mathrm{MHz}$

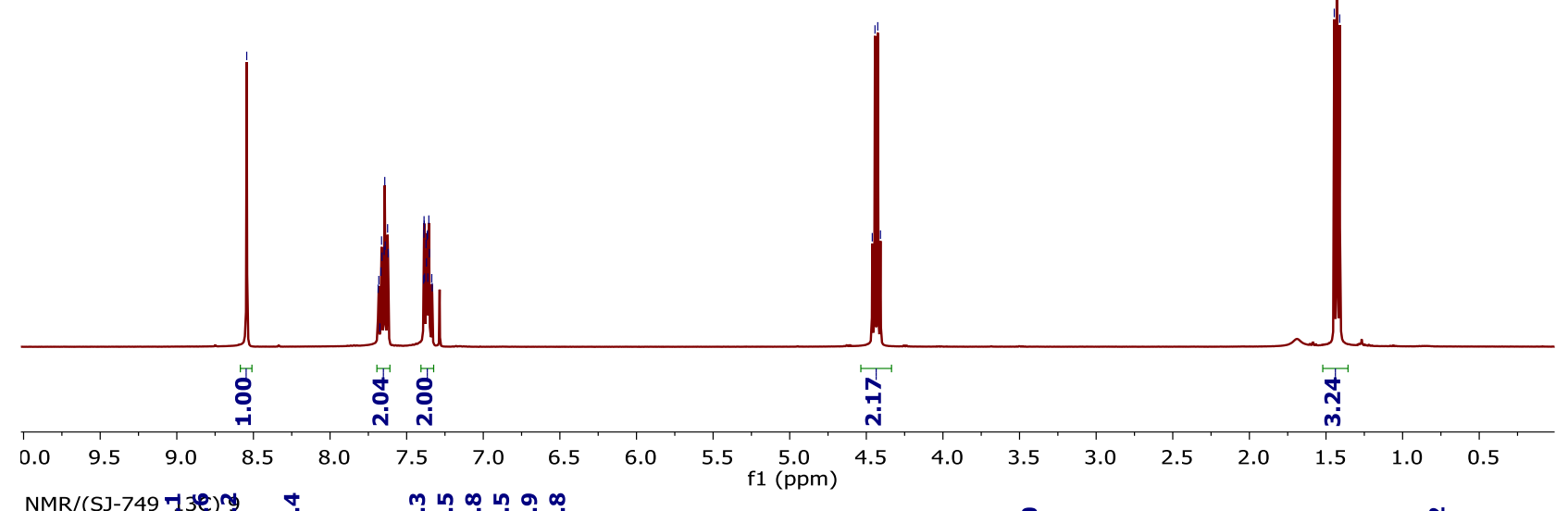

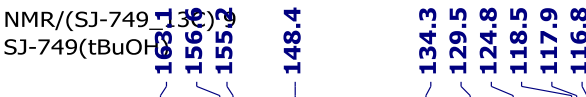

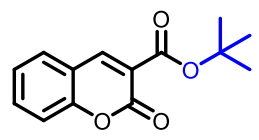

3ag, ${ }^{13} \mathrm{C}\left\{{ }^{1} \mathrm{H}\right\} \mathrm{NMR}$

$\mathrm{CDCl}_{3} 100 \mathrm{MHz}$

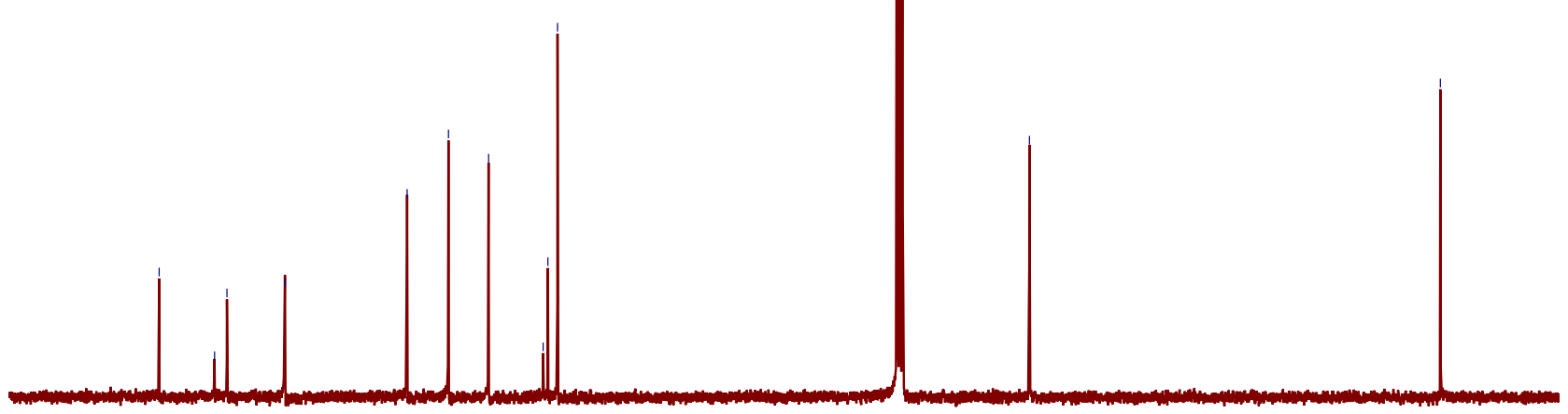

80

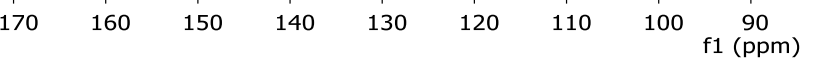

$70 \quad 60$

$50 \quad 40$

$30 \quad 20 \quad 10$ 


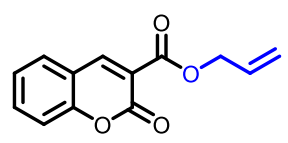

3ah, ${ }^{1} \mathrm{H}$ NMR

$\mathrm{CDCl}_{3} 400 \mathrm{MHz}$

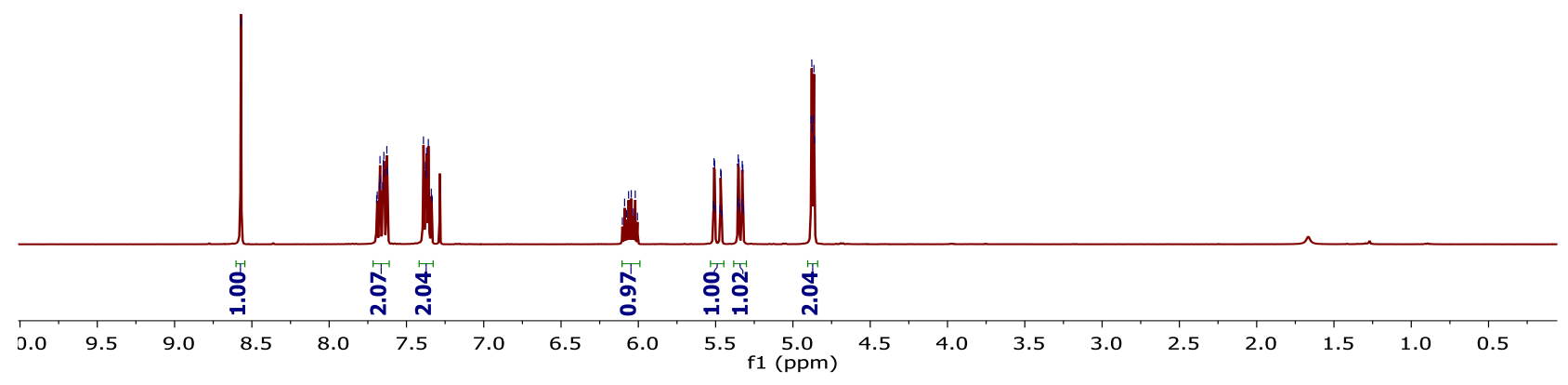

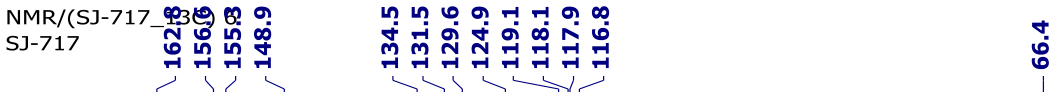

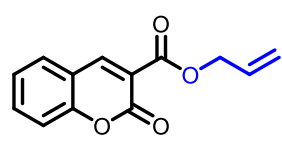

3ah, ${ }^{13} \mathrm{C}\left\{{ }^{1} \mathrm{H}\right\}$ NMR

$\mathrm{CDCl}_{3} 100 \mathrm{MHz}$

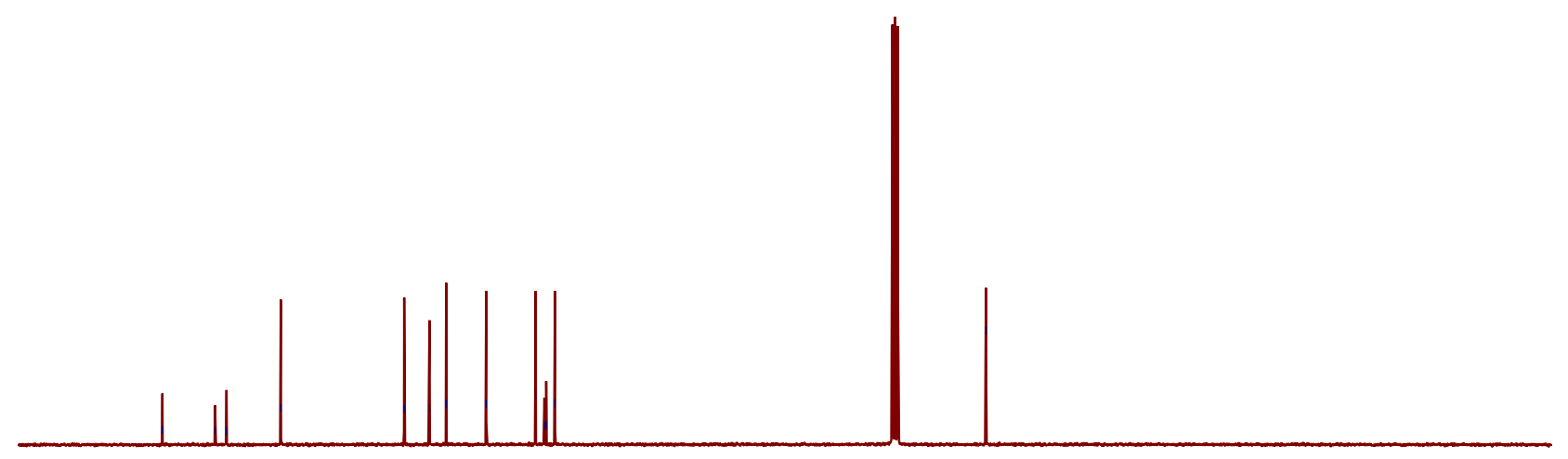

170

$140 \quad 130 \quad 120 \quad 110 \quad 100$

f1 $\stackrel{90}{(\mathrm{ppm})}$ 


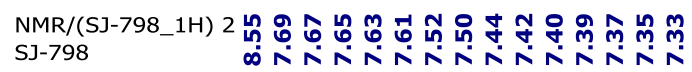

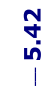
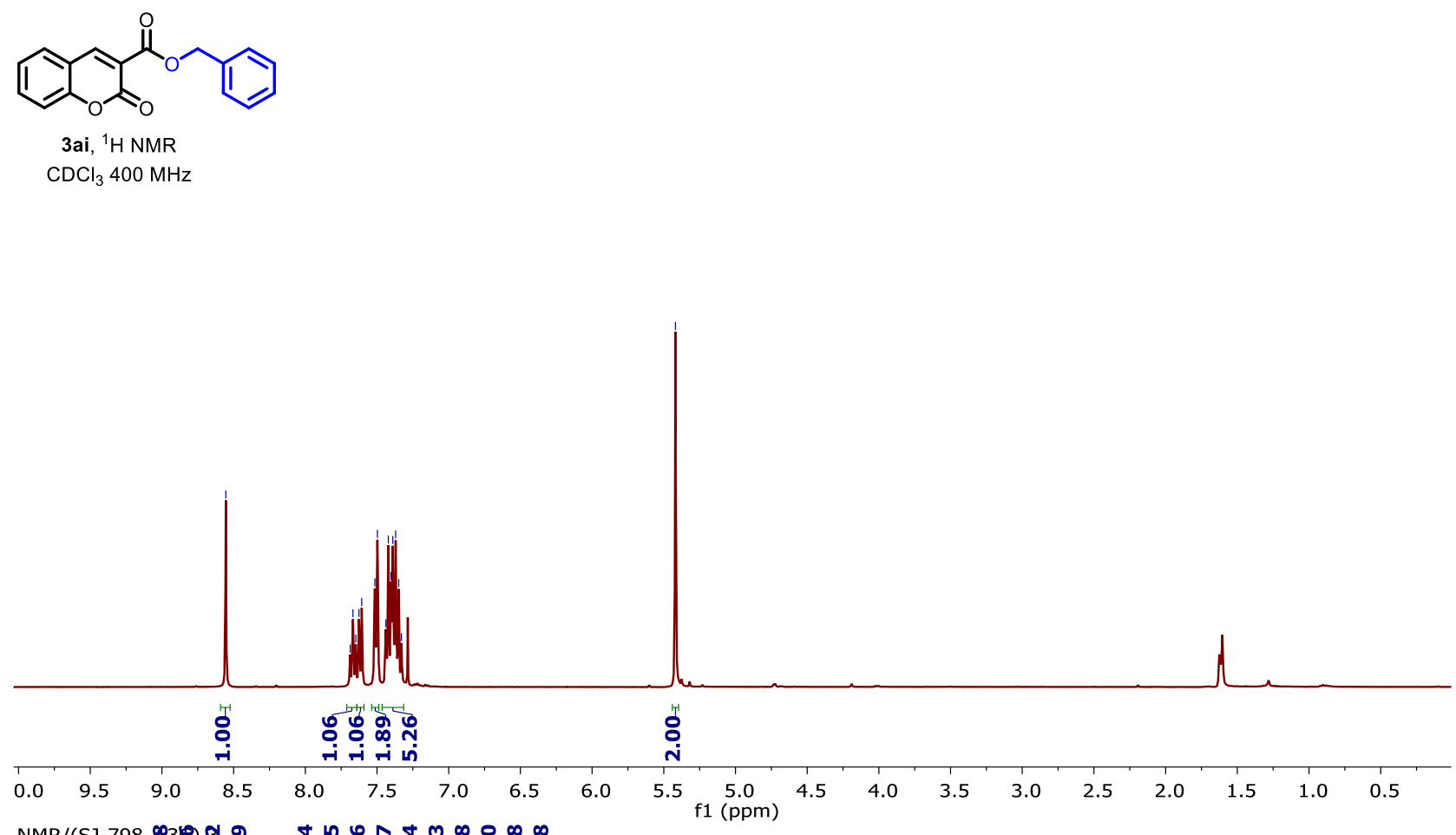

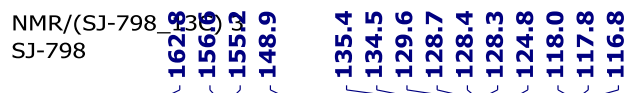

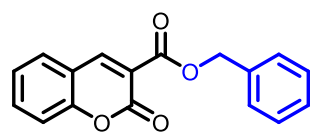

3ai, ${ }^{13} \mathrm{C}\left\{{ }^{1} \mathrm{H}\right\}$ NMR $\mathrm{CDCl}_{3} 100 \mathrm{MHz}$
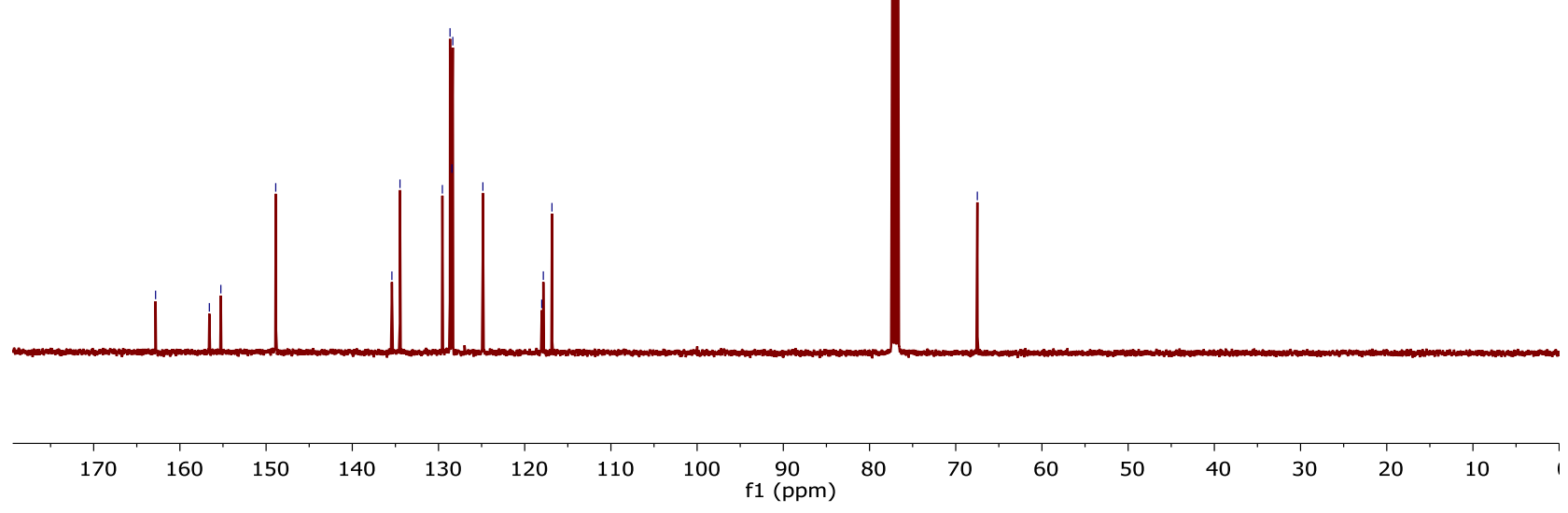

S9 


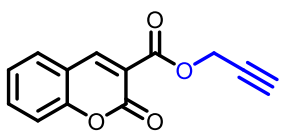

3aj, ${ }^{1} \mathrm{H}$ NMR $\mathrm{CDCl}_{3} 400 \mathrm{MHz}$
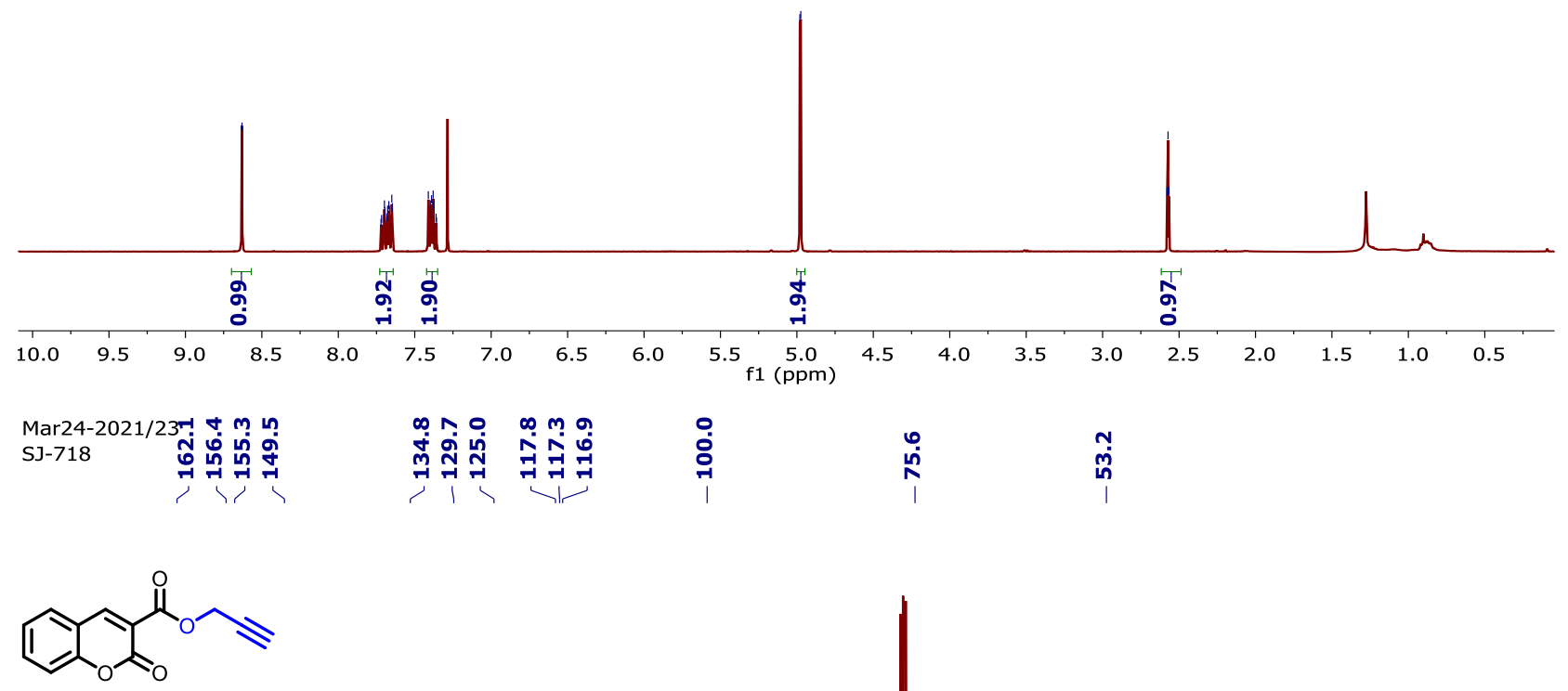

3aj, ${ }^{13} \mathrm{C}\left\{{ }^{1} \mathrm{H}\right\} \mathrm{NMR}$

$\mathrm{CDCl}_{3} 100 \mathrm{MHz}$

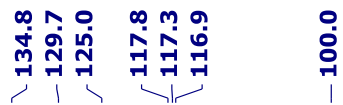

$\stackrel{0}{\stackrel{0}{n}}$

กี่

(

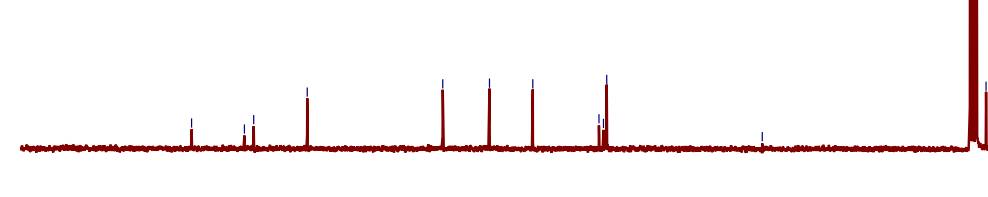

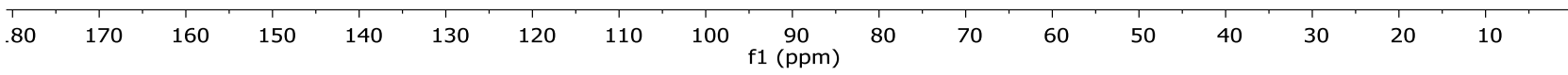




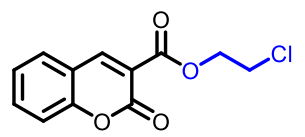

3ak, ${ }^{1} \mathrm{H}$ NMR $\mathrm{CDCl}_{3} 400 \mathrm{MHz}$

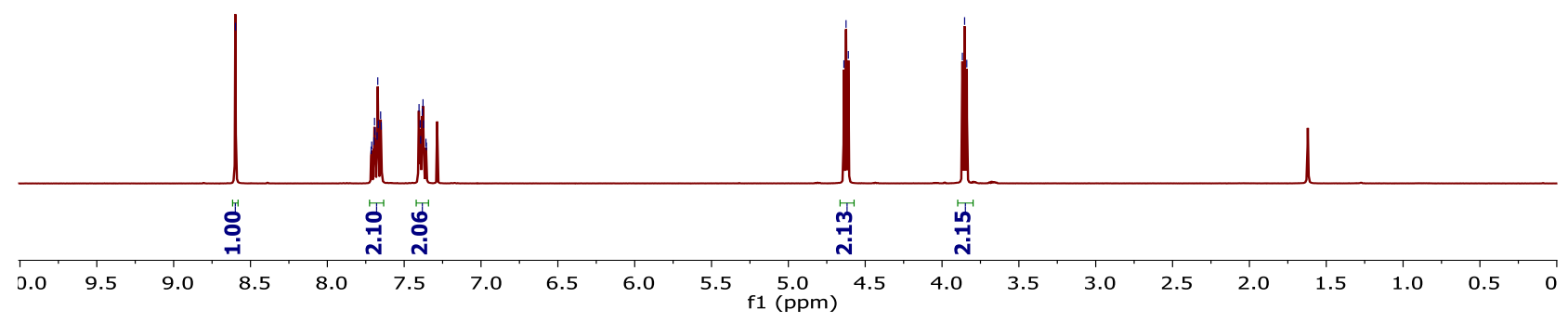

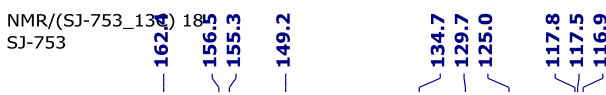

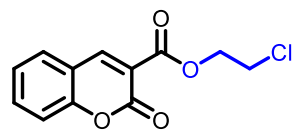

3ak, ${ }^{13} \mathrm{C}\left\{{ }^{1} \mathrm{H}\right\} \mathrm{NMR}$

$\mathrm{CDCl}_{3} 100 \mathrm{MHz}$

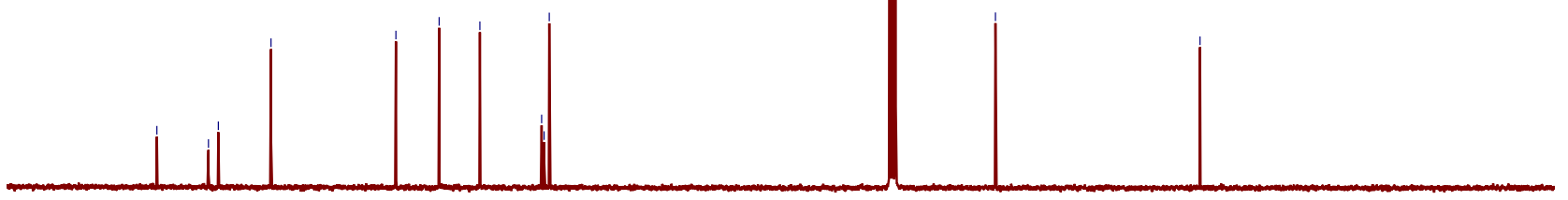

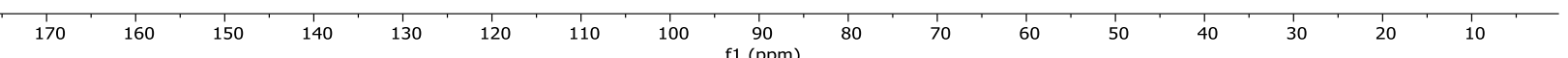




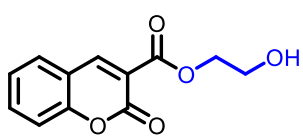

3al, ${ }^{1} \mathrm{H}$ NMR

$\mathrm{CDCl}_{3} 400 \mathrm{MHz}$

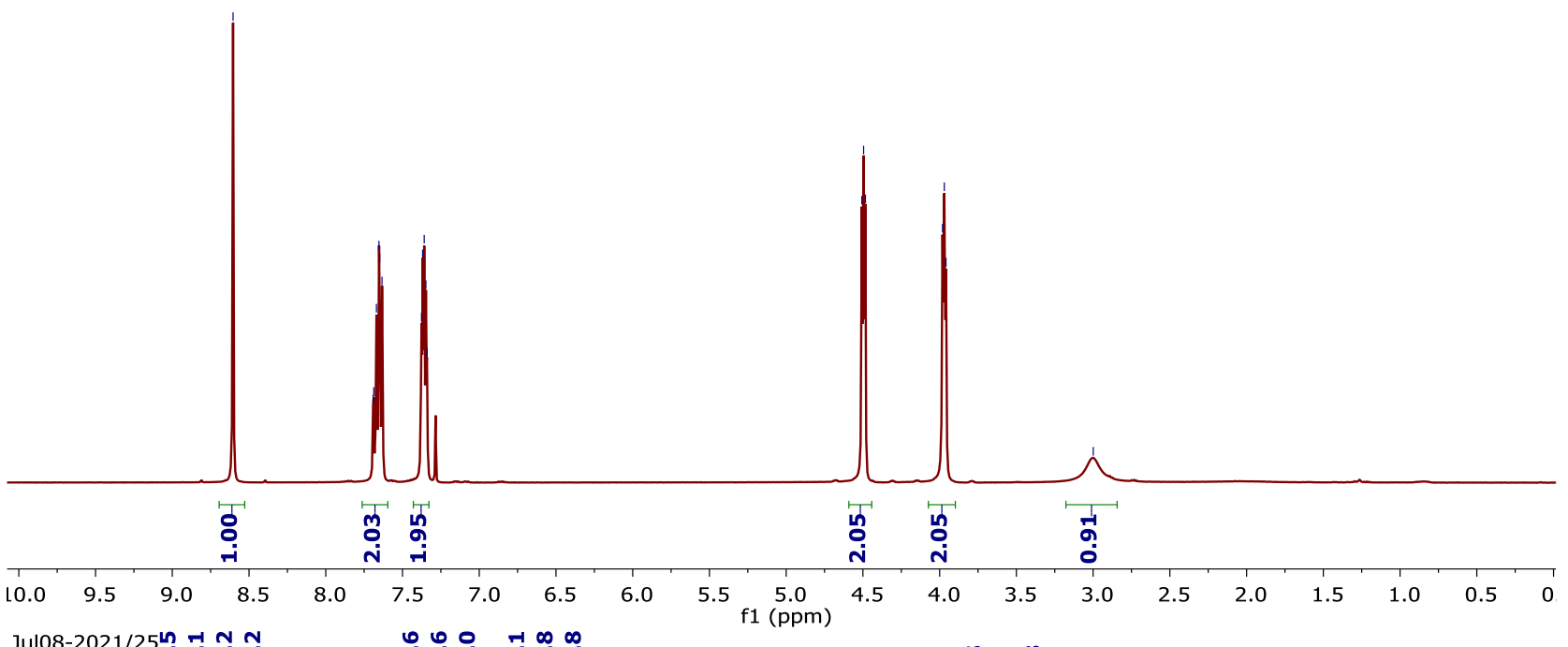

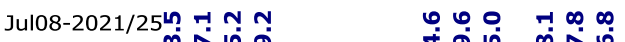

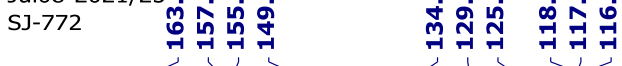

人,

$\stackrel{n}{n} \stackrel{\infty}{8}$

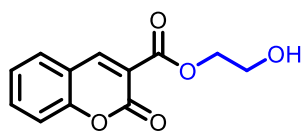

3al, ${ }^{13} \mathrm{C}\left\{{ }^{1} \mathrm{H}\right\}$ NMR

$\mathrm{CDCl}_{3} 100 \mathrm{MHz}$
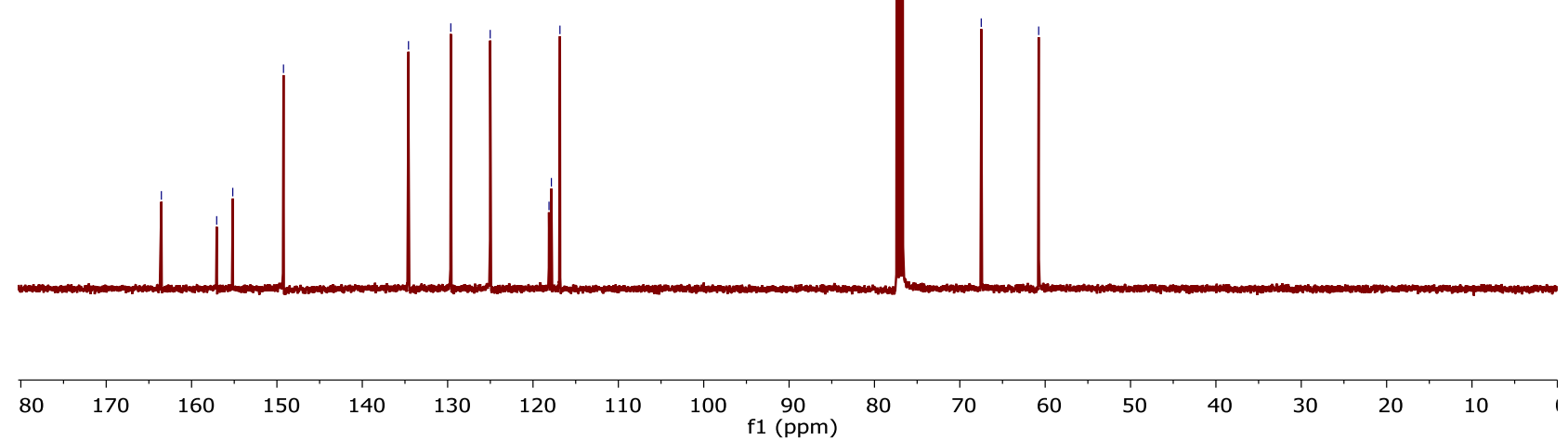

80

40

$30 \quad 20 \quad 10$ 
<smiles>CCCCCCOC(=O)c1ccccc1</smiles>

3bd, ${ }^{1} \mathrm{H}$ NMR

$\mathrm{CDCl}_{3} 400 \mathrm{MHz}$

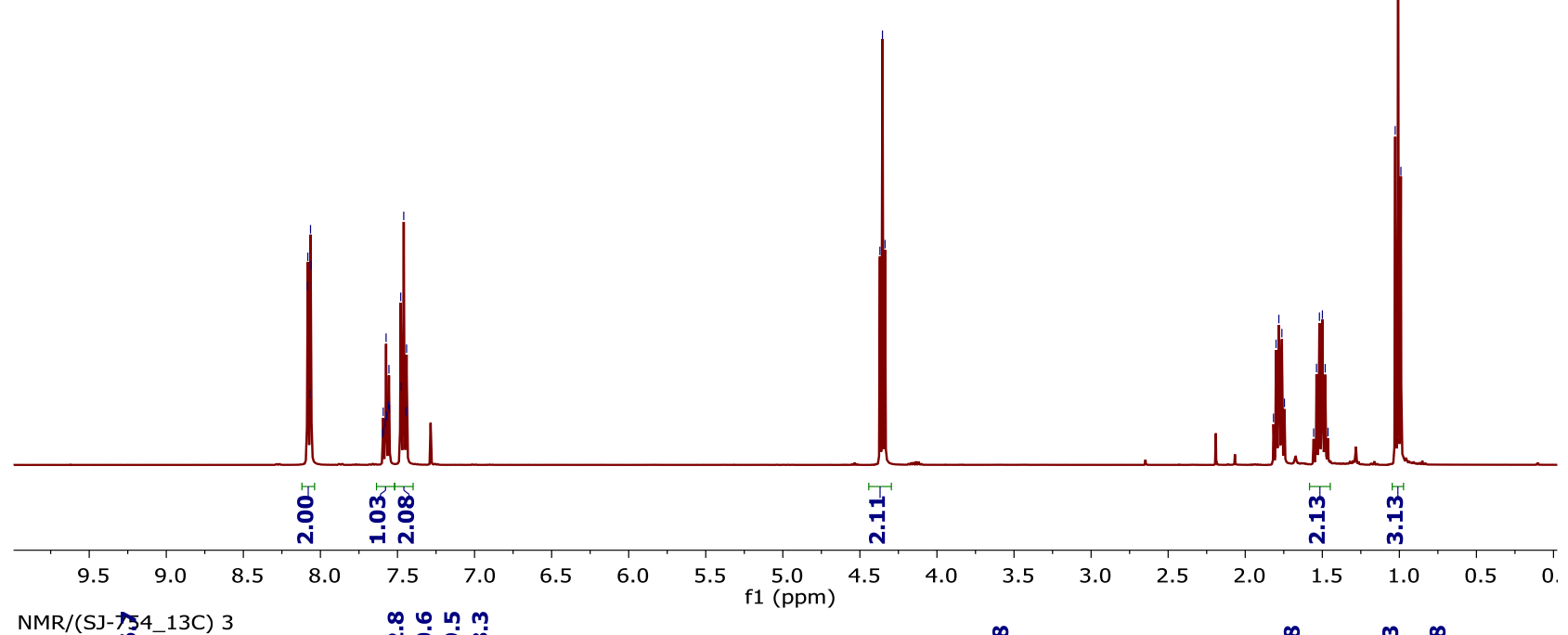
SJ-754(BE: Z)

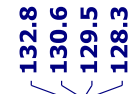
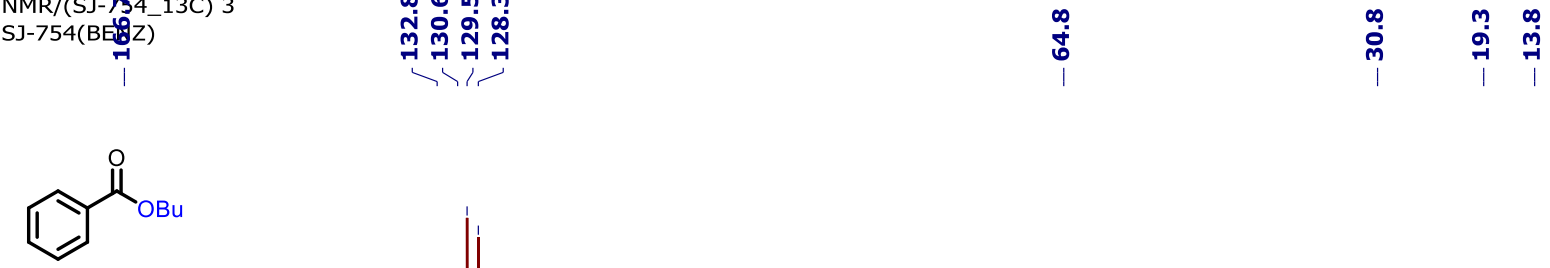

3bd, ${ }^{13} \mathrm{C}\left\{{ }^{1} \mathrm{H}\right\}$ NMR

$\mathrm{CDCl}_{3} 100 \mathrm{MHz}$

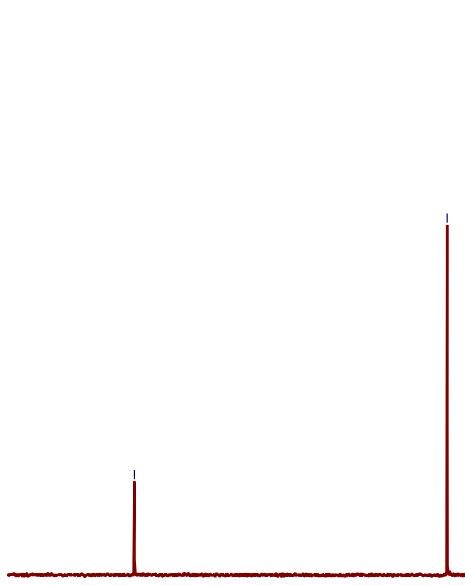

$$
80
$$

80170
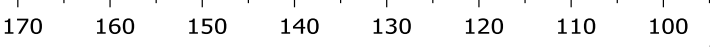

f1 (ppm)
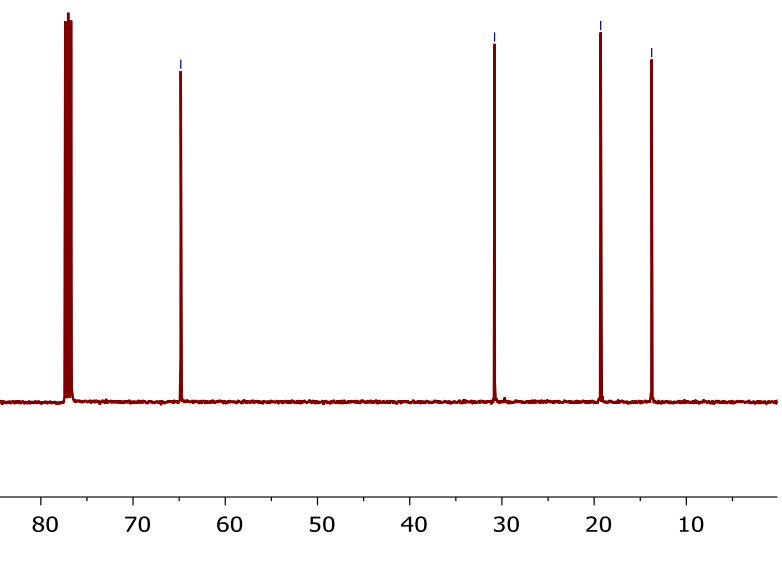

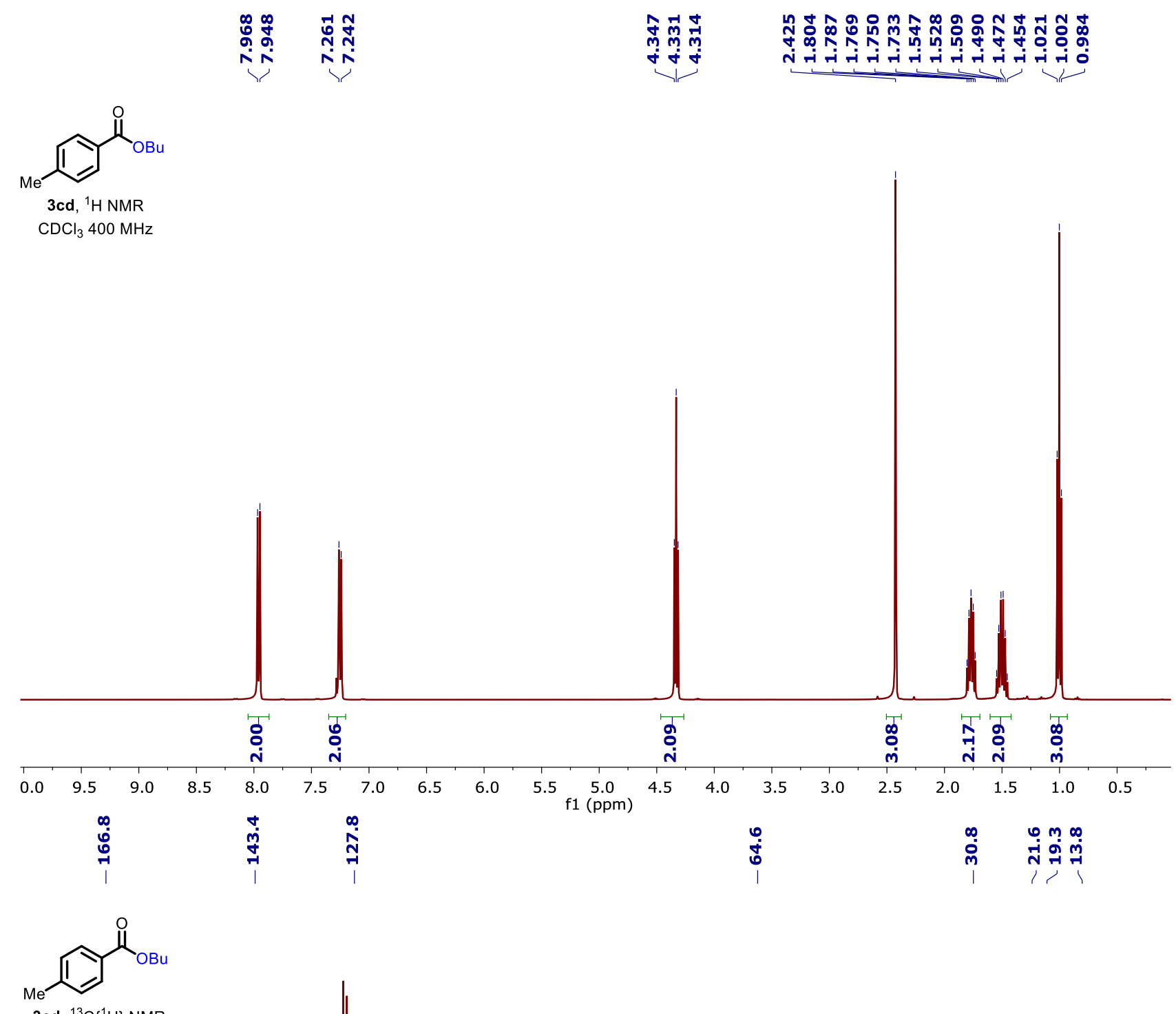

3cd, ${ }^{13} \mathrm{C}\left\{{ }^{1} \mathrm{H}\right\}$ NMR

$\mathrm{CDCl}_{3} 100 \mathrm{MHz}$
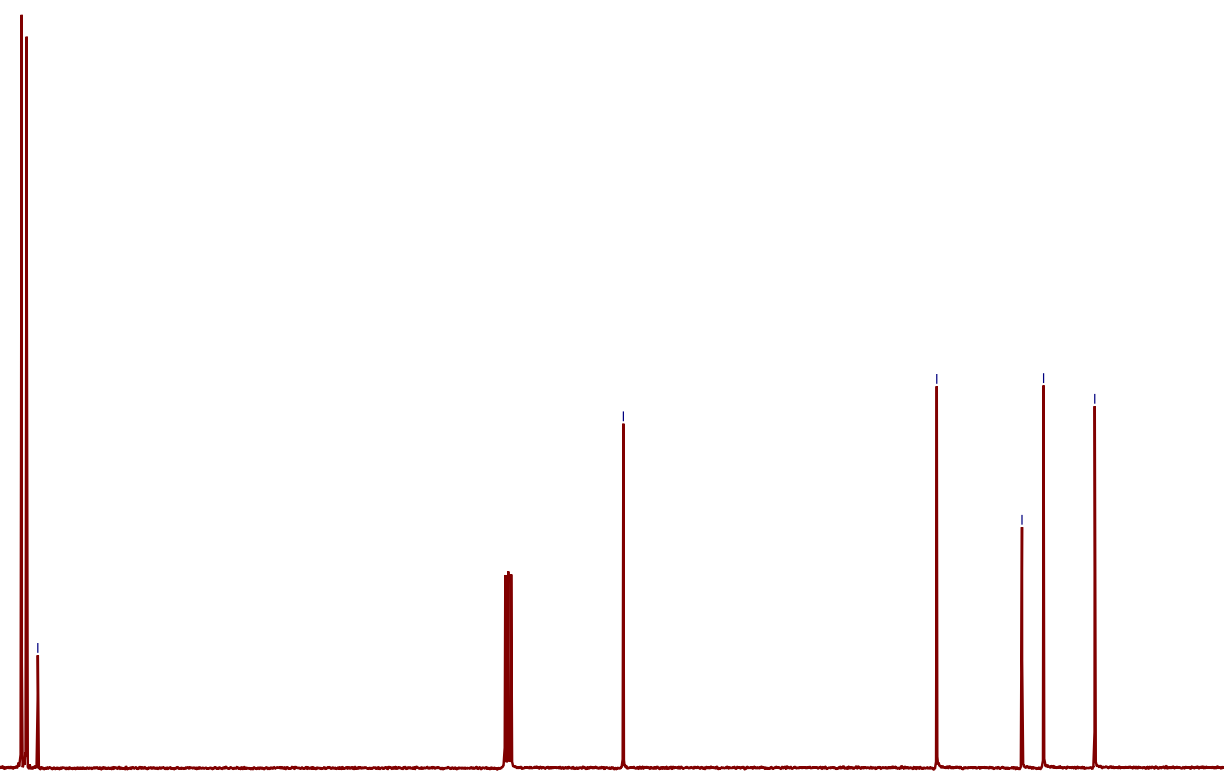

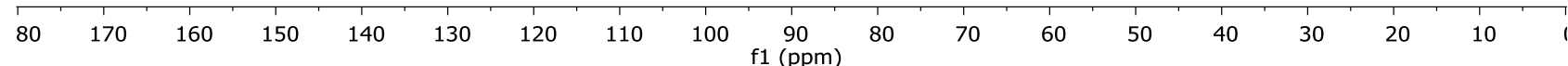




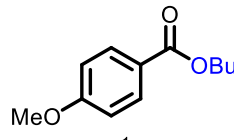

3dd, ${ }^{1} \mathrm{H}$ NMR

$\mathrm{CDCl}_{3} 400 \mathrm{MHz}$
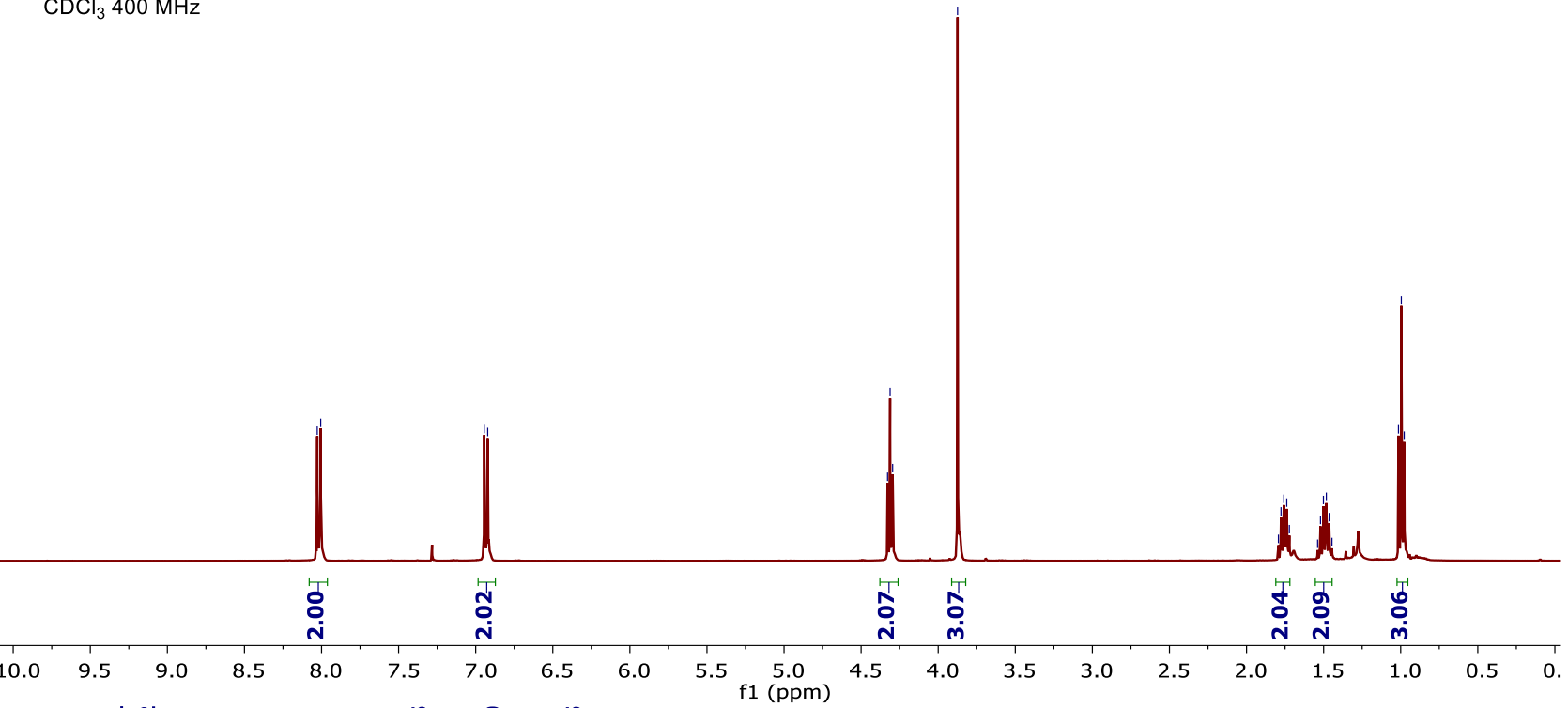

$\mathrm{NMR} /\left(\mathrm{SJ}-\mathrm{T} 5 \mathrm{~N}_{13 \mathrm{C}} 51\right.$

SJ-735 웅

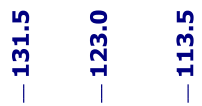

品苫

品

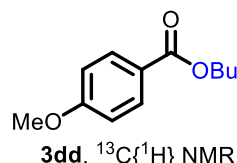

3dd, ${ }^{13} \mathrm{C}\left\{{ }^{1} \mathrm{H}\right\}$ NMR

$\mathrm{CDCl}_{3} 100 \mathrm{MHz}$

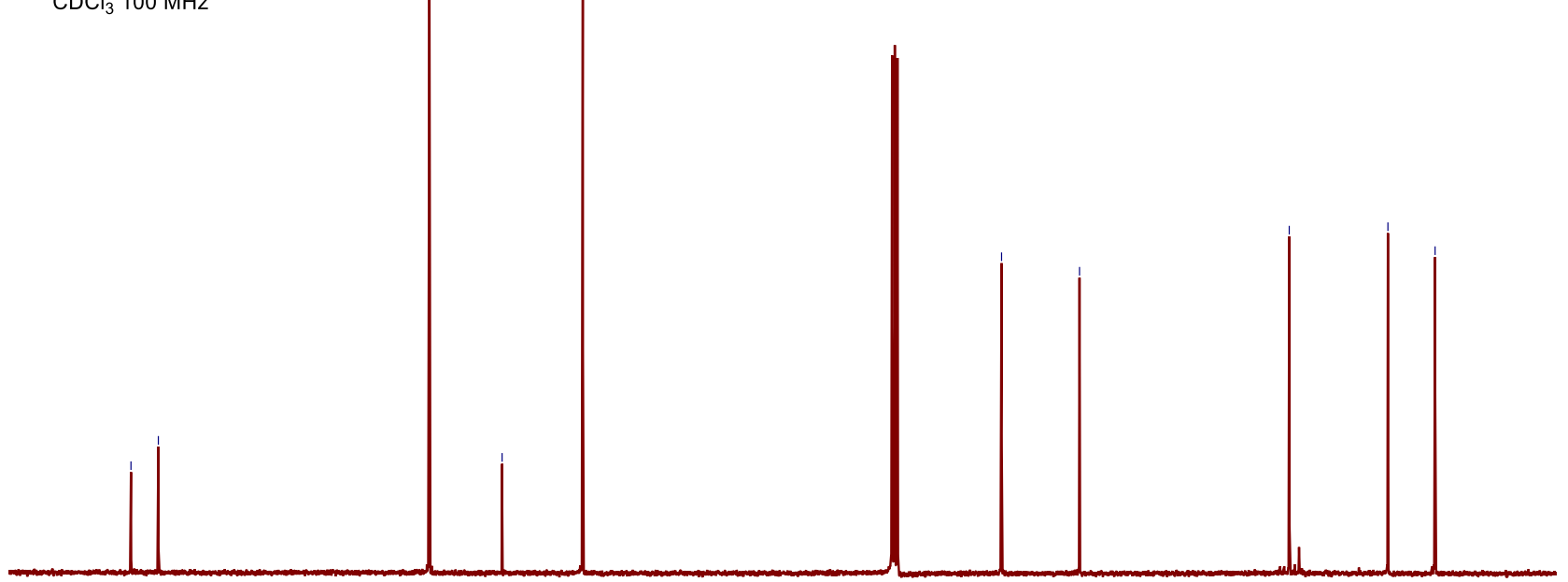

80

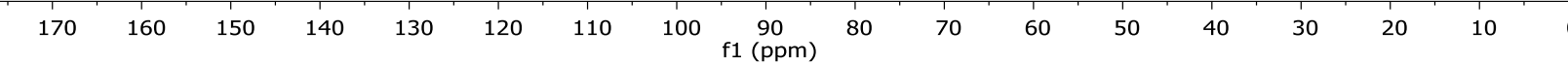




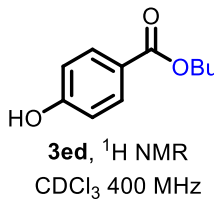

$\mathrm{CDCl}_{3} 400 \mathrm{MHz}$
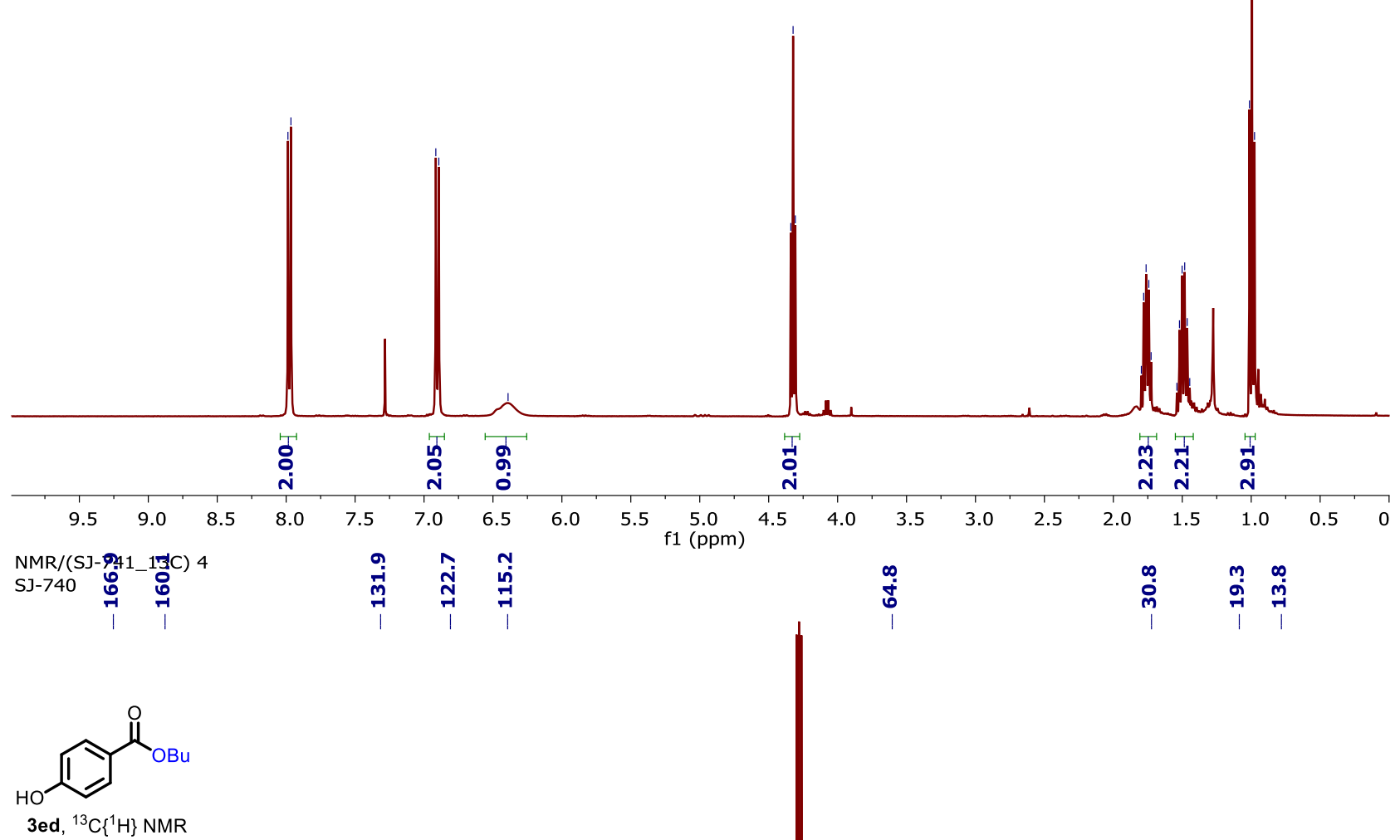

$\mathrm{CDCl}_{3} 100 \mathrm{MHz}$

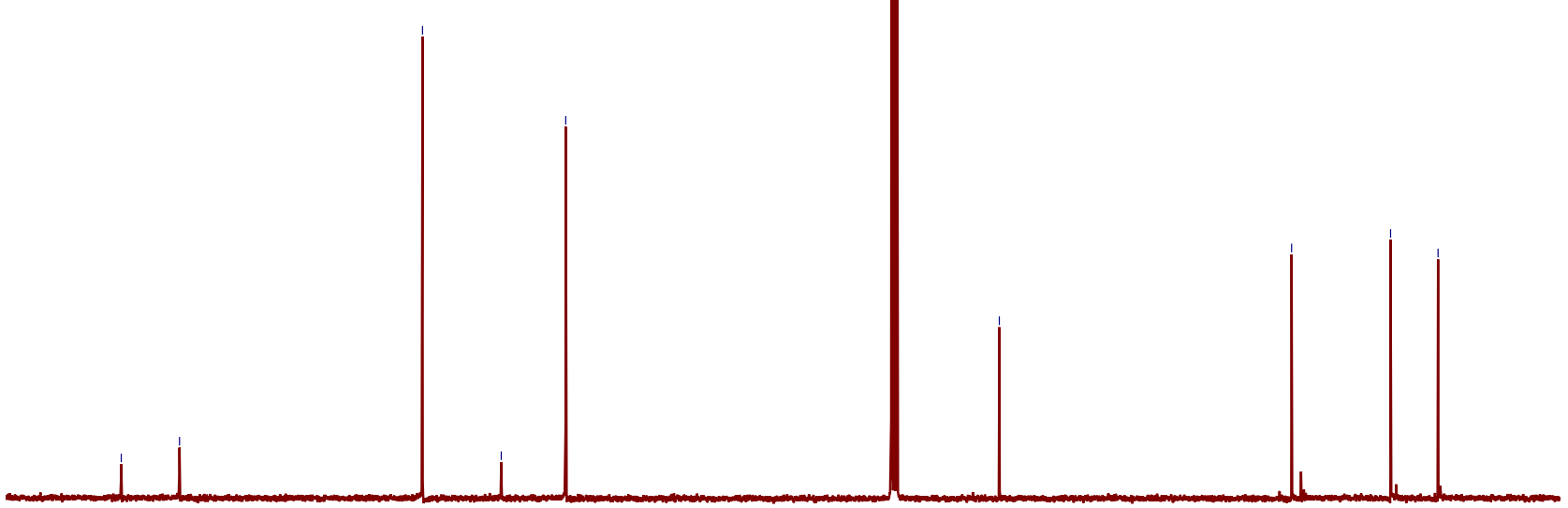




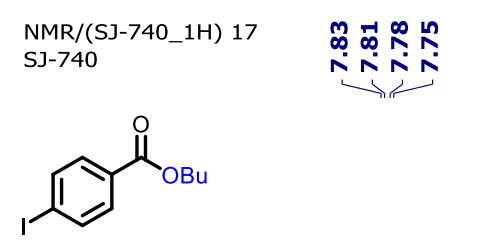

3fd, ${ }^{1} \mathrm{H}$ NMR

$\mathrm{CDCl}_{3} 400 \mathrm{MHz}$
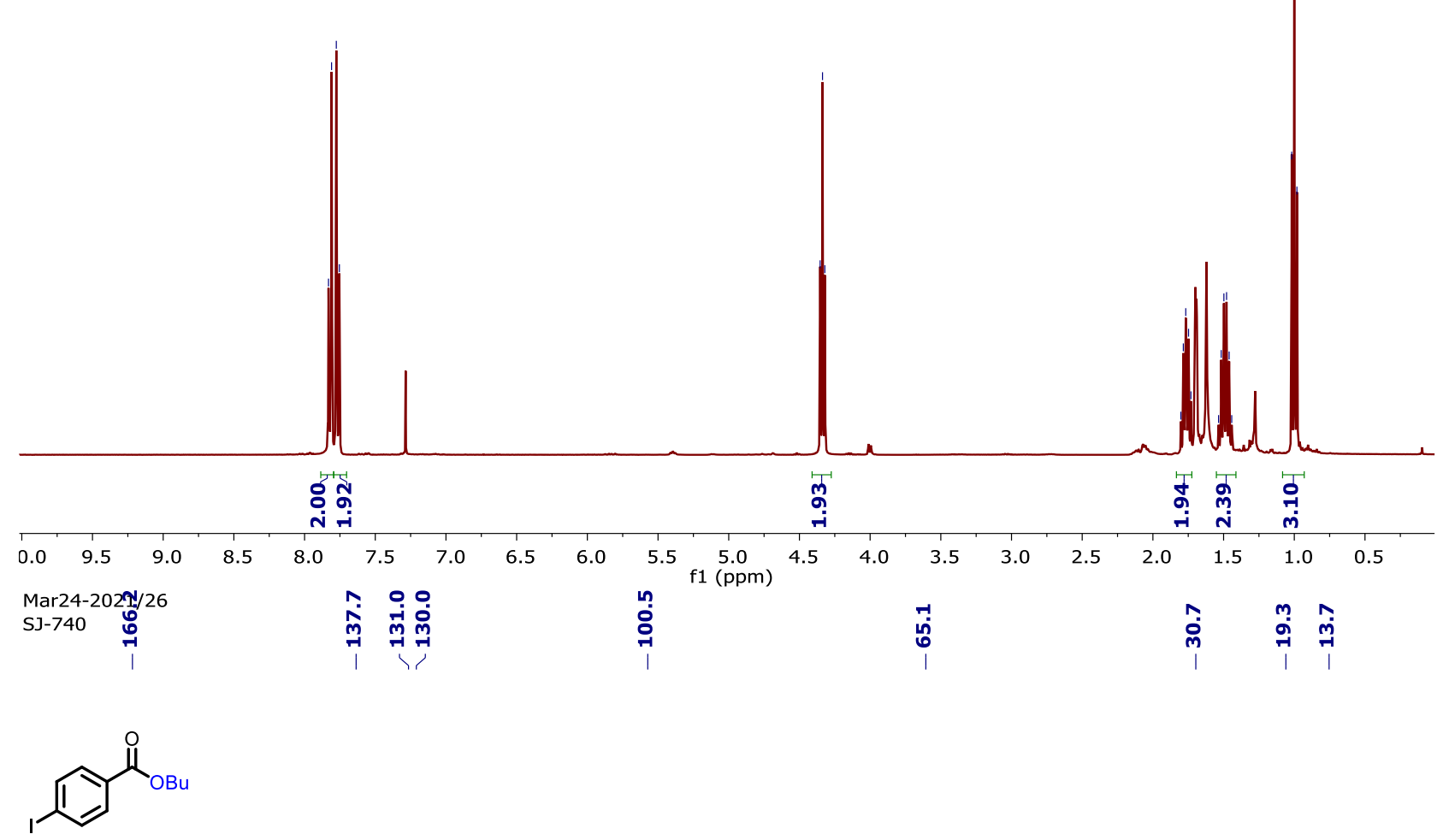

3fd, ${ }^{13} \mathrm{C}\left\{{ }^{1} \mathrm{H}\right\}$ NMR

$\mathrm{CDCl}_{3} 100 \mathrm{MHz}$

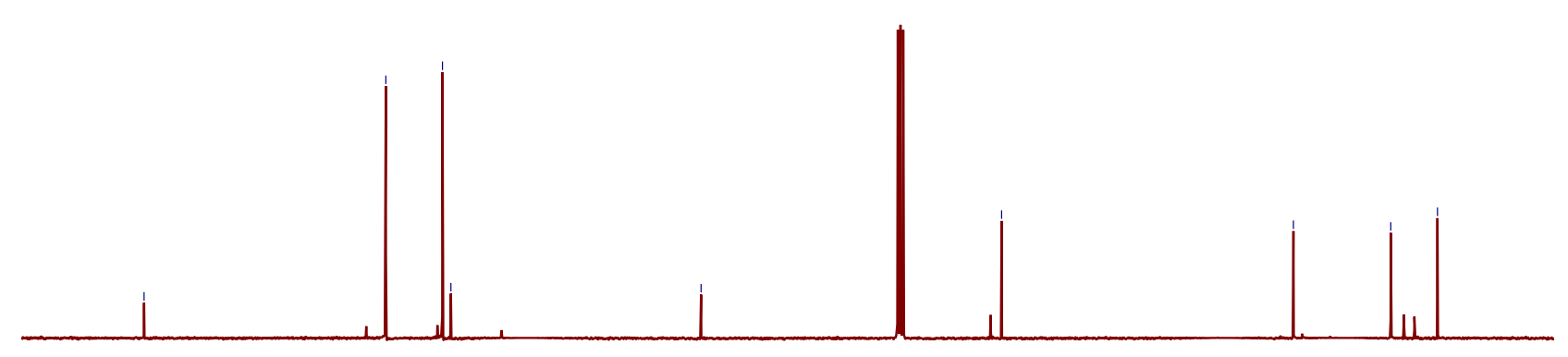

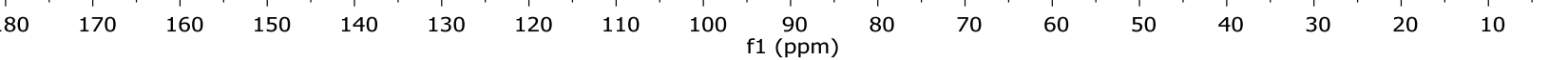


กิ

$\infty \infty^{\infty} \infty \infty^{\infty} \infty \infty^{\infty} \infty \infty^{\infty} \infty \infty^{\infty} \infty$

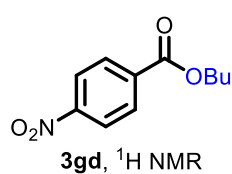

$\mathrm{CDCl}_{3} 400 \mathrm{MHz}$

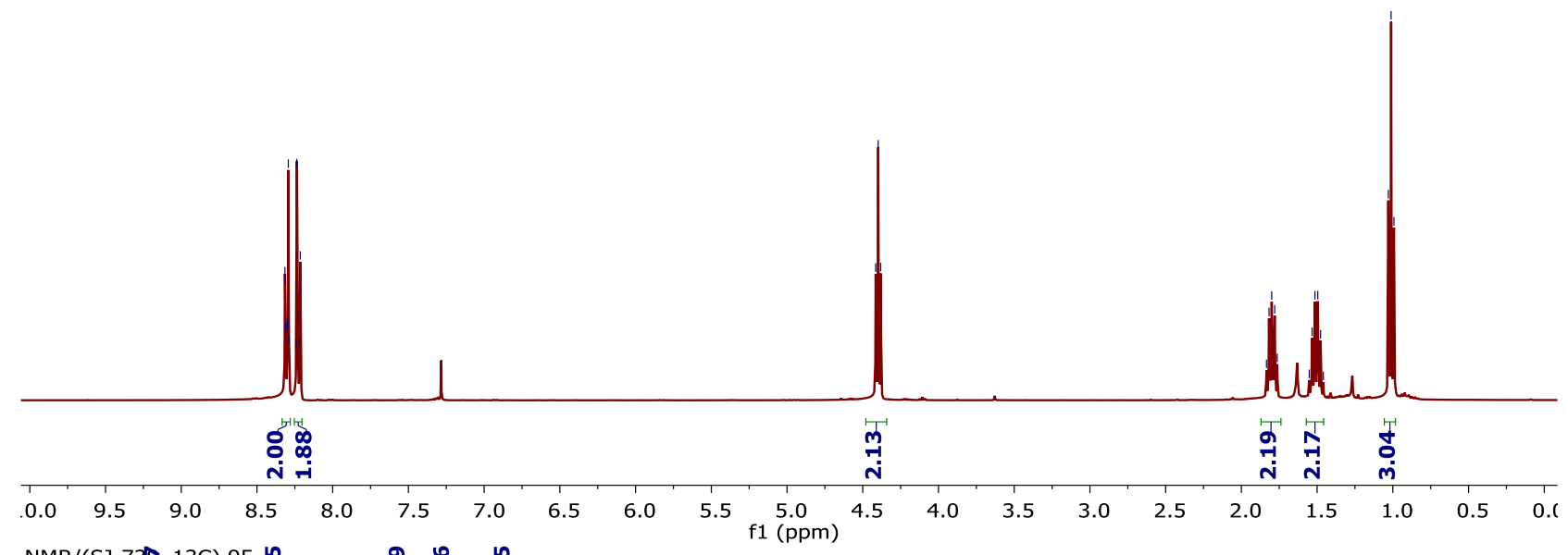

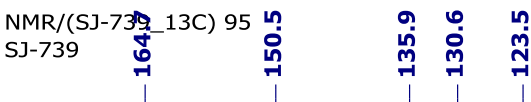

กீ.

ஸั่

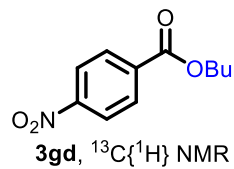

3gd, ${ }^{13} \mathrm{C}\left\{{ }^{1} \mathrm{H}\right\}$ NMR

$\mathrm{CDCl}_{3} 100 \mathrm{MHz}$
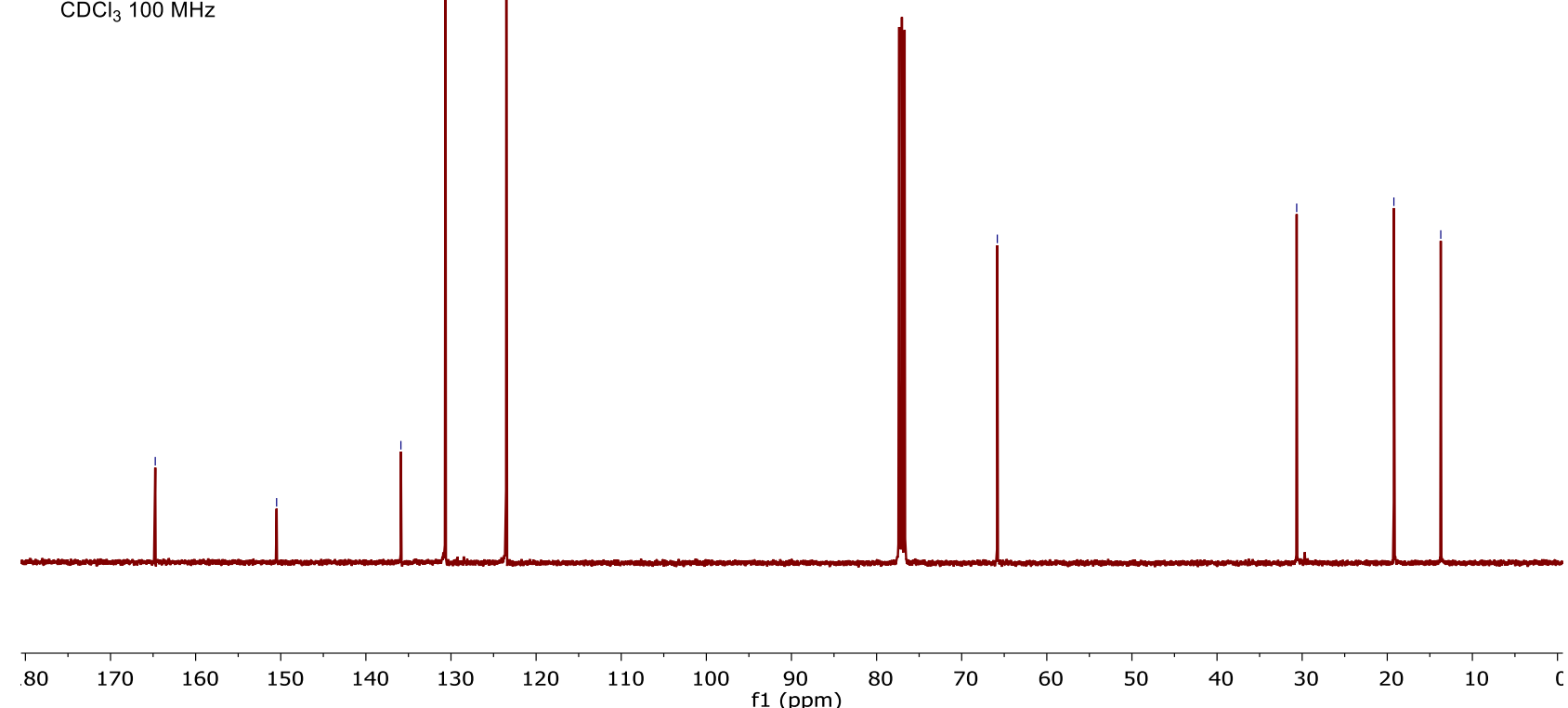

f1 $\stackrel{90}{(\mathrm{ppm})}$

80

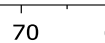




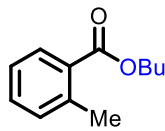

3hd, ${ }^{1} \mathrm{H}$ NMR

$\mathrm{CDCl}_{3} 400 \mathrm{MHz}$

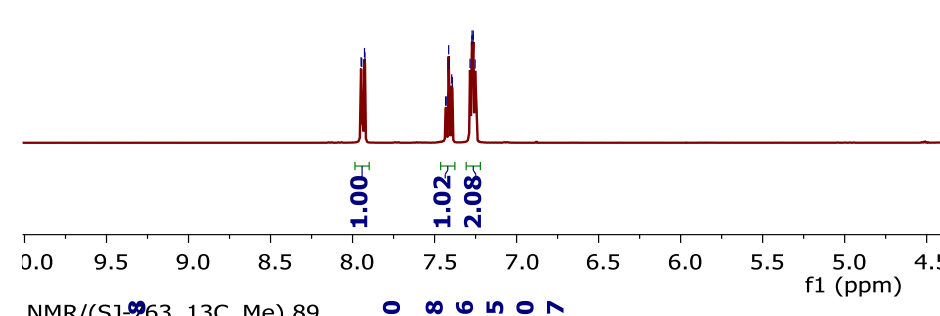

NMR/(SJ-9.63_13C_Me) 89 SJ-763

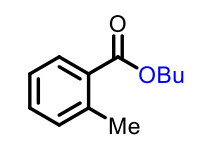

3hd, ${ }^{13} \mathrm{C}\left\{{ }^{1} \mathrm{H}\right\}$ NMR

$\mathrm{CDCl}_{3} 100 \mathrm{MHz}$

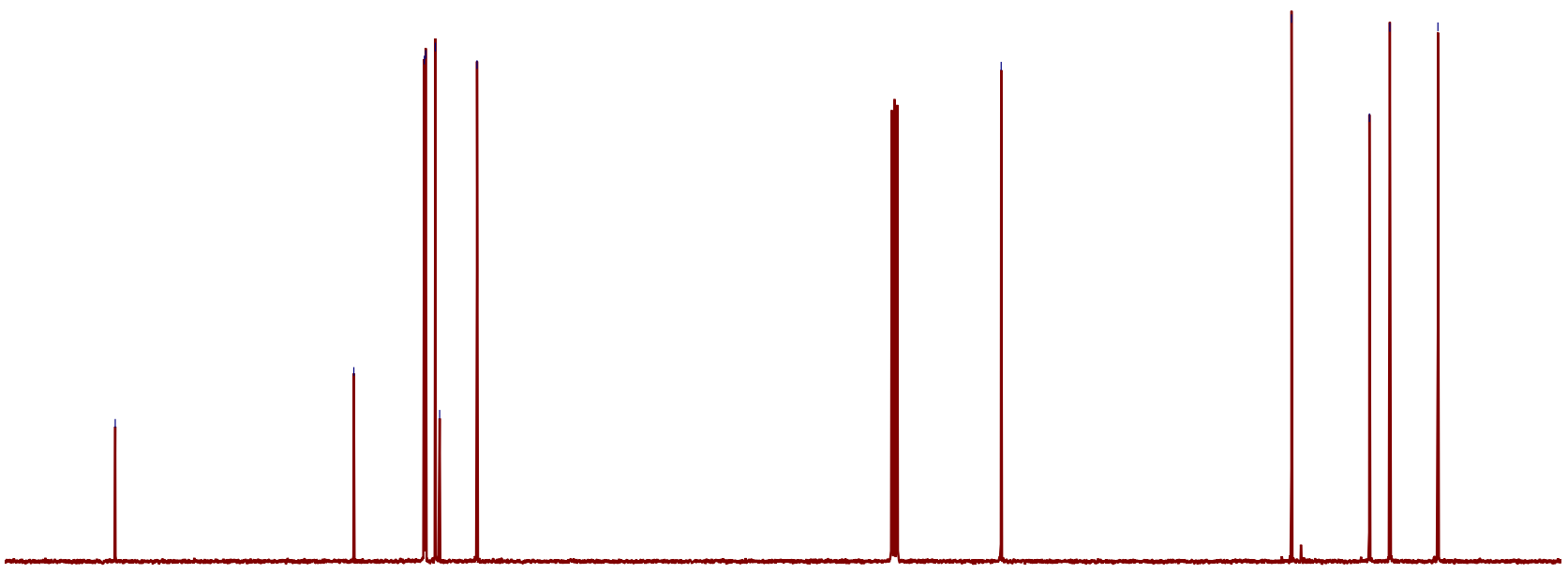

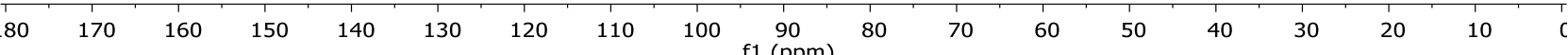




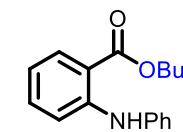

3id, ${ }^{1} \mathrm{H}$ NMR

$\mathrm{CDCl}_{3} 400 \mathrm{MHz}$

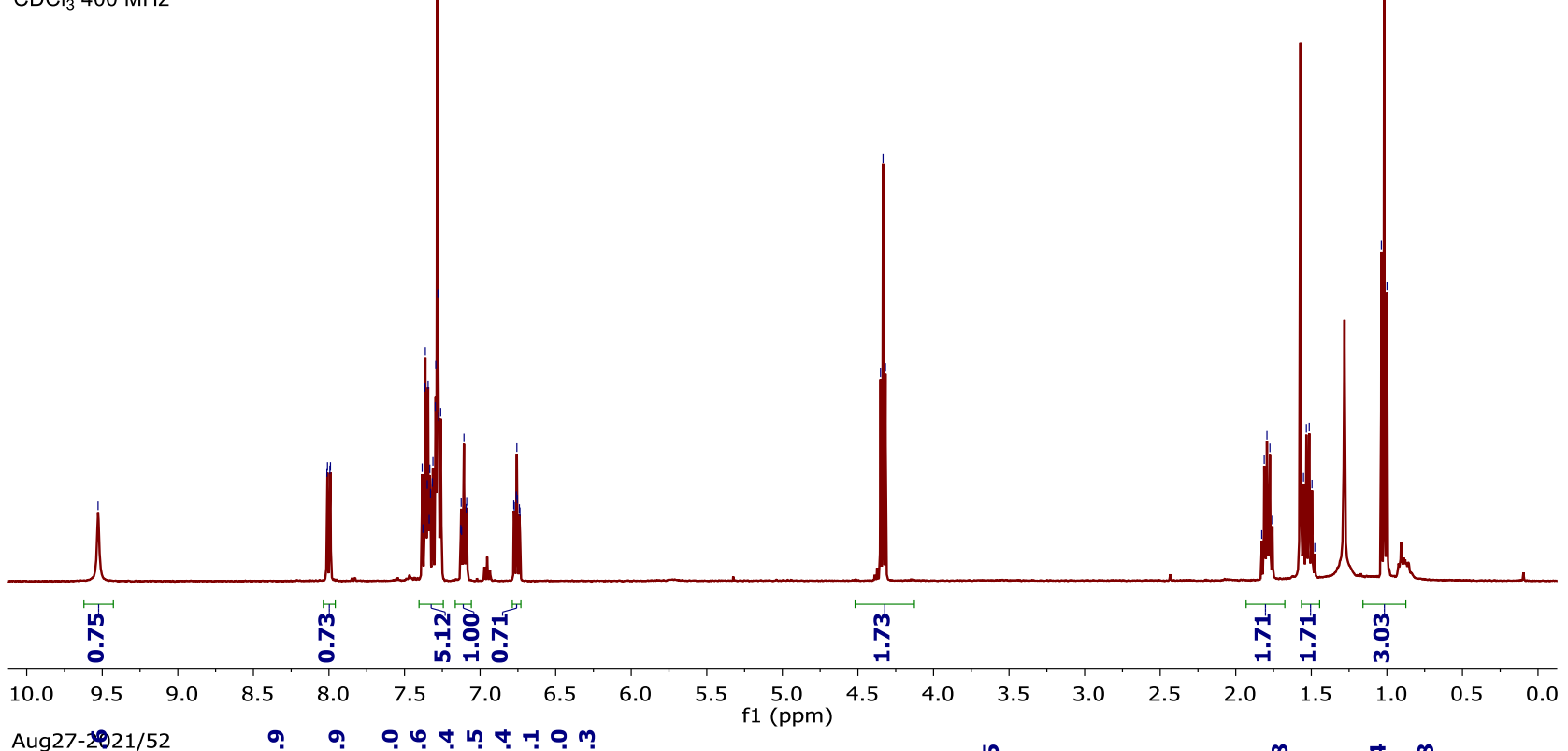

Aug27

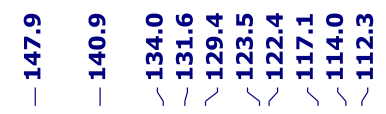

桨

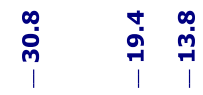

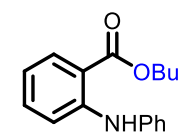

3id, ${ }^{13} \mathrm{C}\left\{{ }^{1} \mathrm{H}\right\}$ NMR

$\mathrm{CDCl}_{3} 100 \mathrm{MHz}$

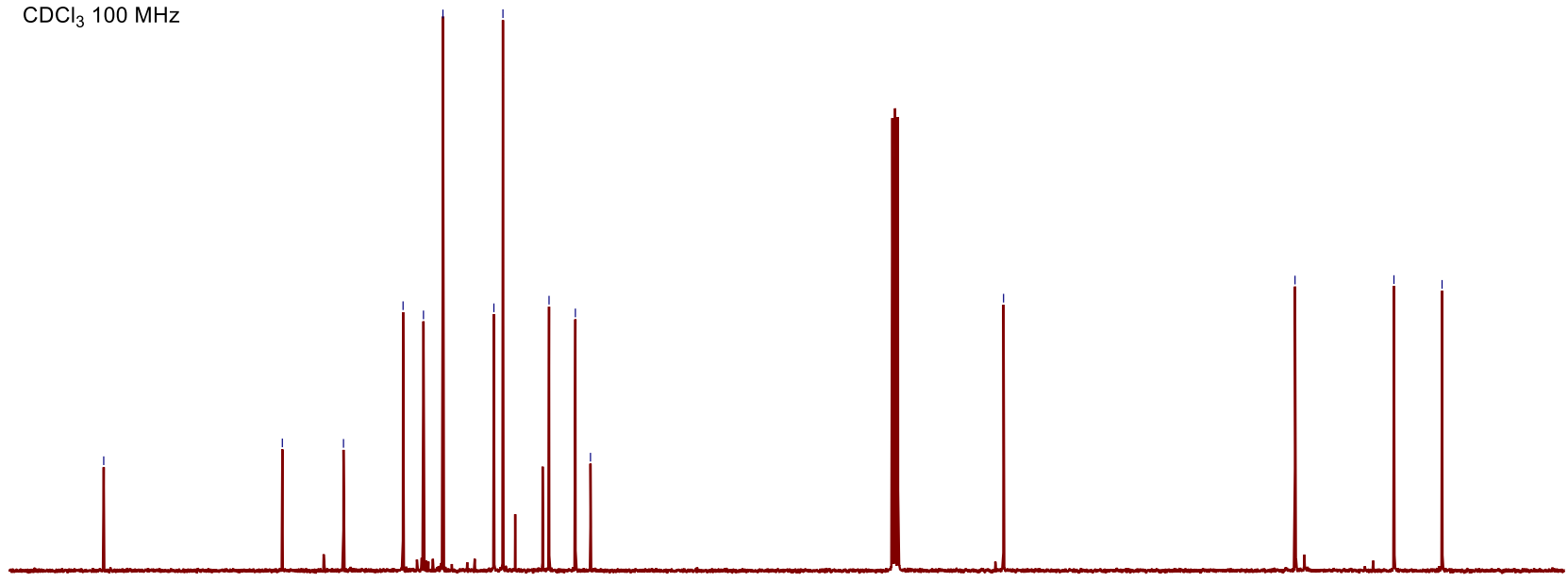

$150 \quad 140$

$110 \quad 100$

90

80

$+60$

\begin{tabular}{llll|}
$50 \quad 40$ & 30 & 20 & 10
\end{tabular}


Jul07-2021/10

SJ-762

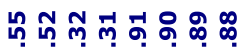

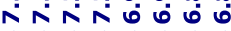

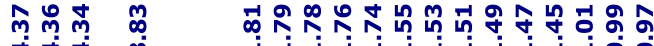

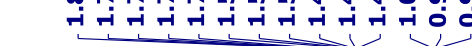

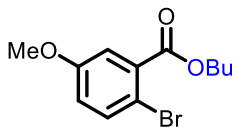

3jd, ${ }^{1} \mathrm{H}$ NMR

$\mathrm{CDCl}_{3} 400 \mathrm{MHz}$

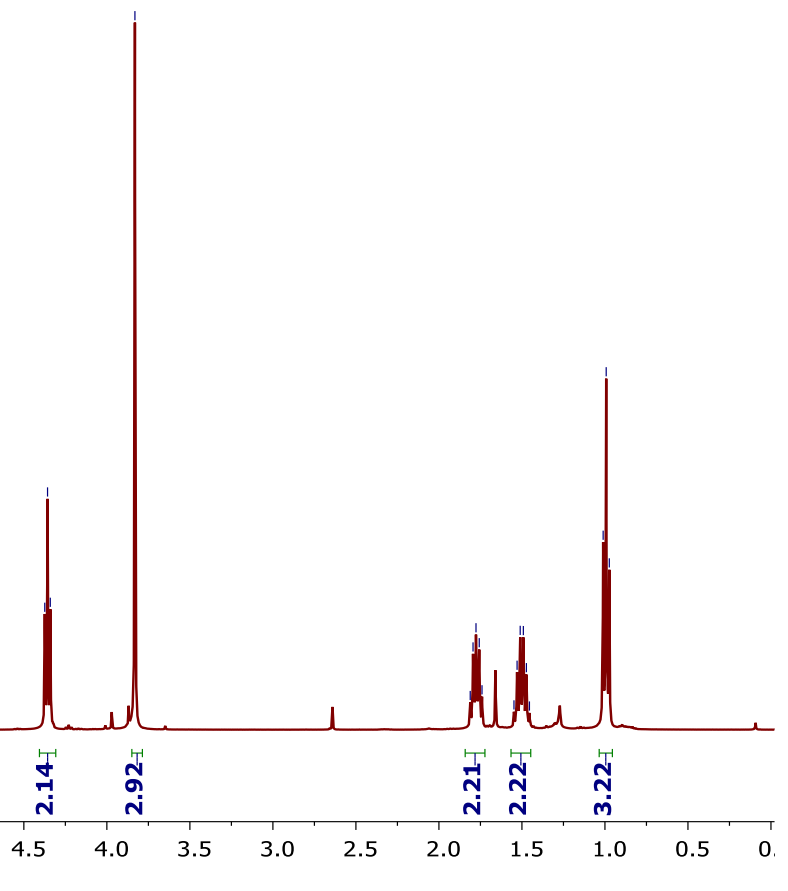

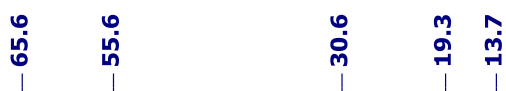

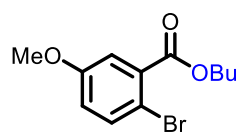

3jd, ${ }^{13} \mathrm{C}\left\{{ }^{1} \mathrm{H}\right\}$ NMR

$\mathrm{CDCl}_{3} 100 \mathrm{MHz}$

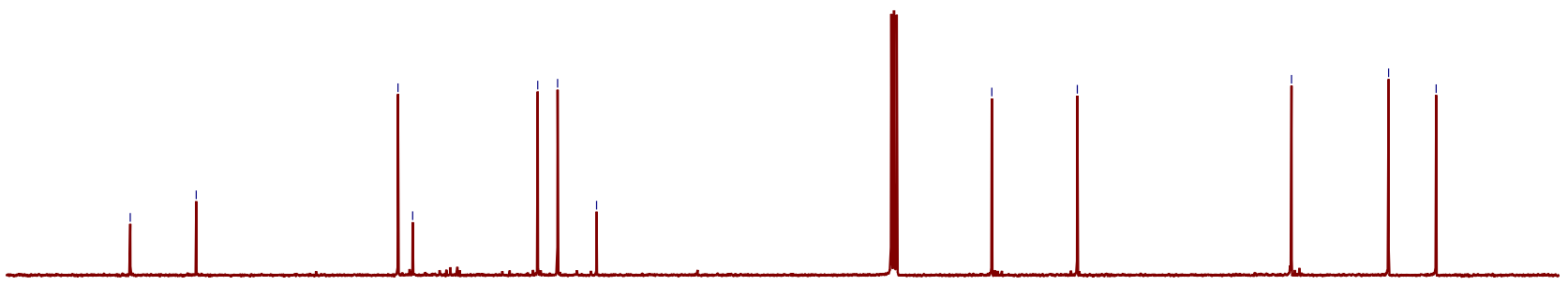

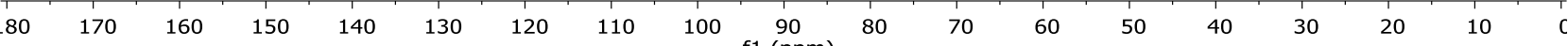




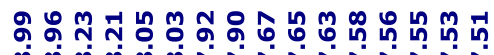

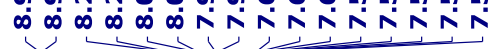

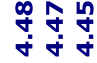

ฉి

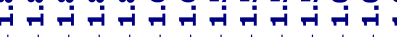

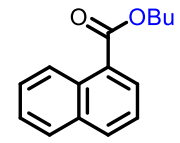

3kd, ${ }^{1} \mathrm{H}$ NMR

$\mathrm{CDCl}_{3} 400 \mathrm{MHz}$
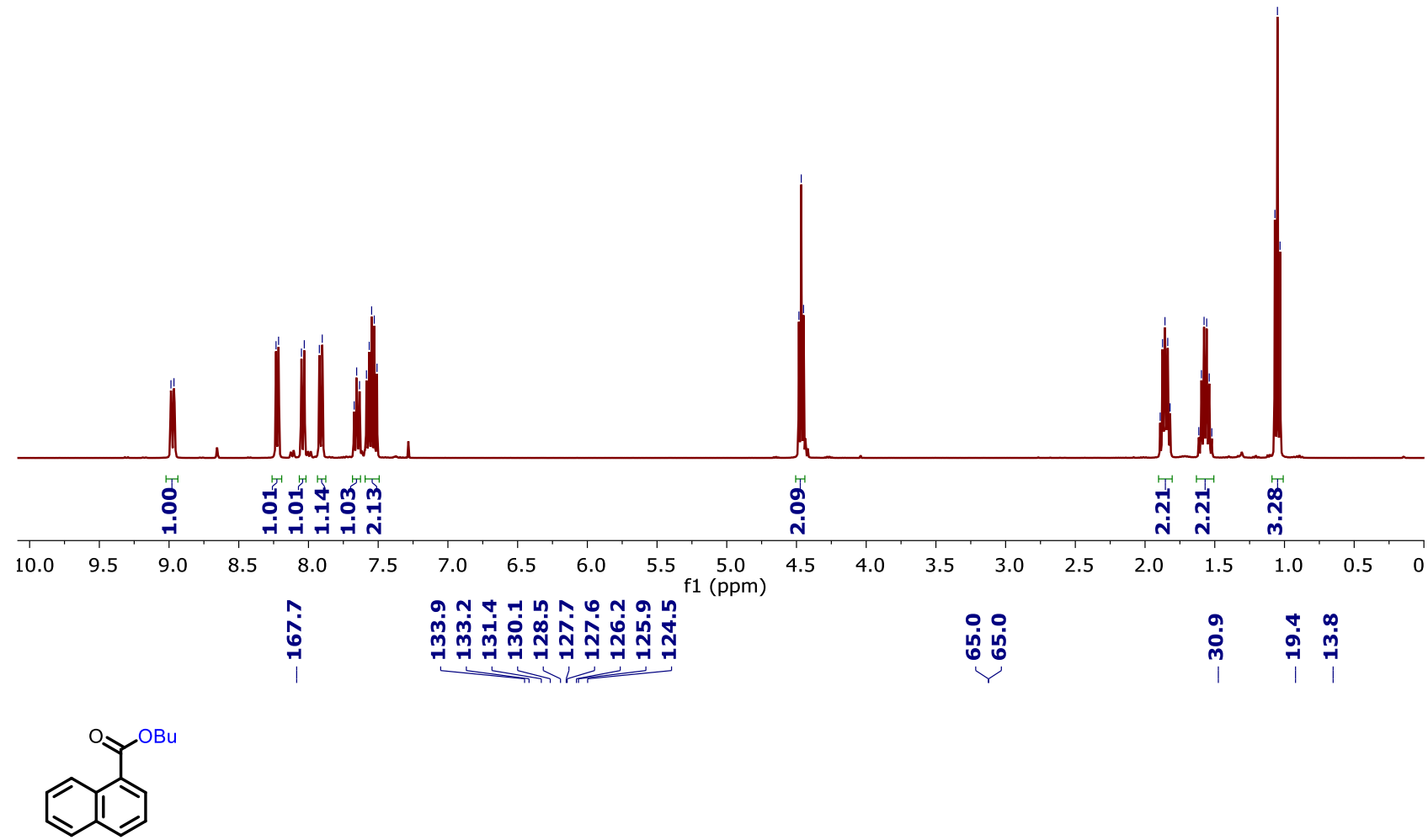

3kd, ${ }^{13} \mathrm{C}\left\{{ }^{1} \mathrm{H}\right\}$ NMR

$\mathrm{CDCl}_{3} 100 \mathrm{MHz}$

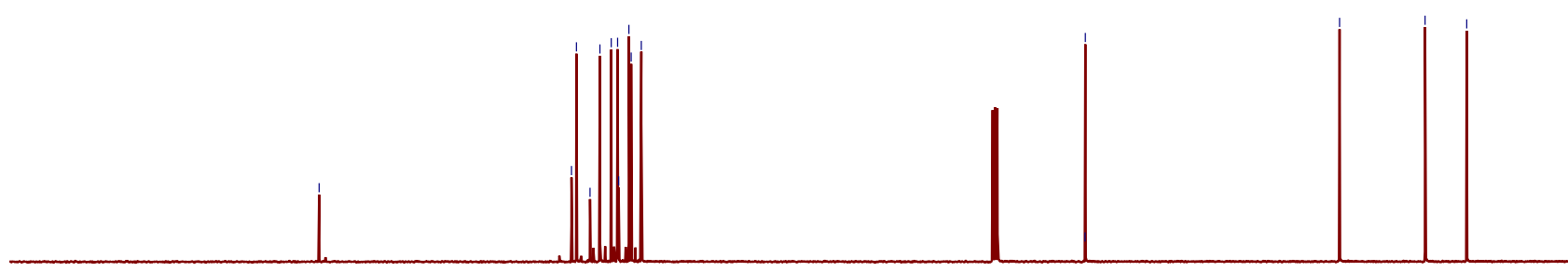

200

$\begin{array}{llllllllll}190 & 180 & 170 & 160 & 150 & 140 & 130 & 120 & \begin{array}{c}110 \\ \mathrm{f} 1(\mathrm{ppm})\end{array}\end{array}$

$\begin{array}{lllllllll}90 & 80 & 70 & 60 & 50 & 40 & 30 & 20 & 10\end{array}$ 


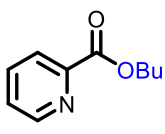

3ld, ${ }^{1} \mathrm{H}$ NMR

$\mathrm{CDCl}_{3} 400 \mathrm{MHz}$
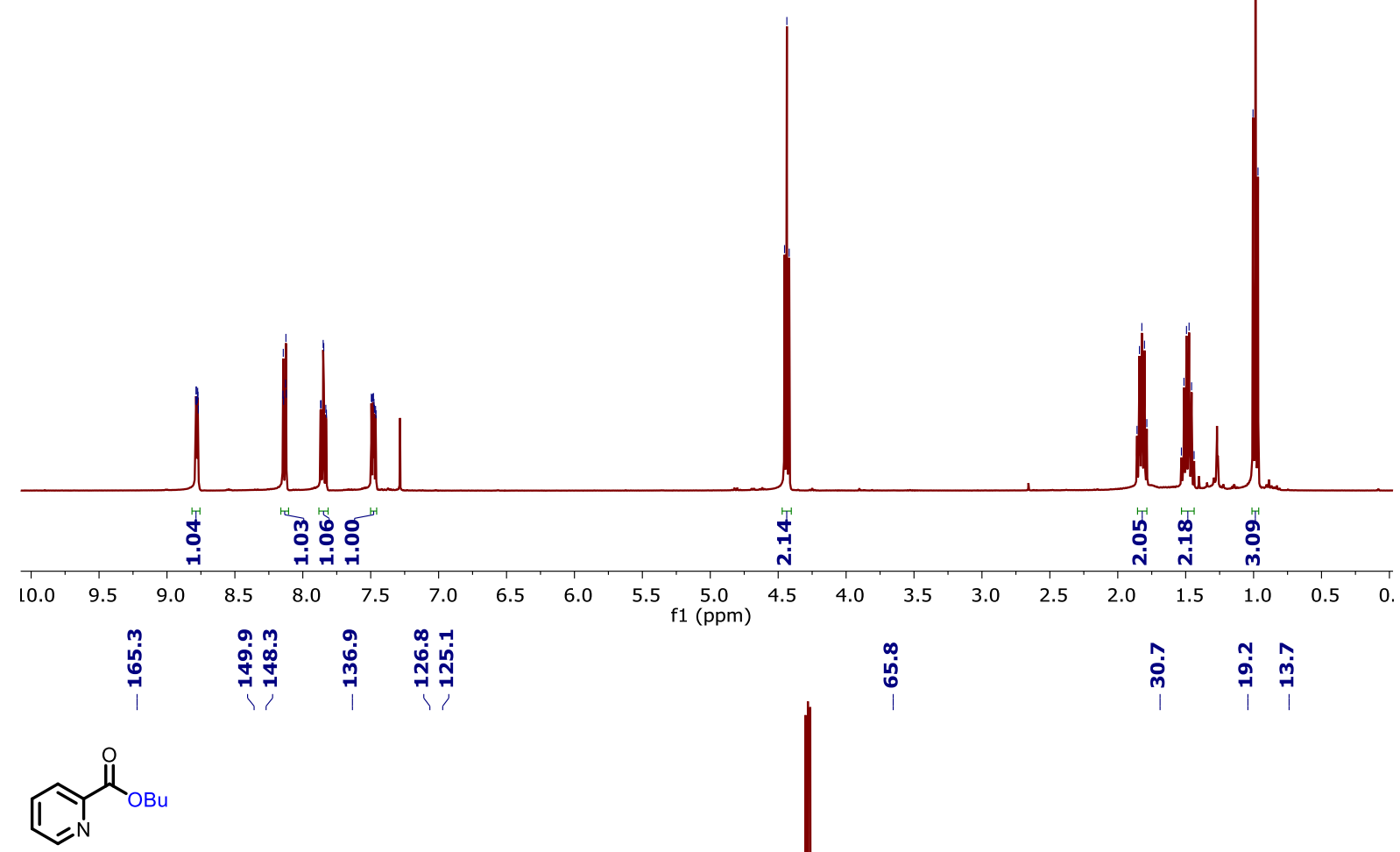

3Id, ${ }^{13} \mathrm{C}\left\{{ }^{1} \mathrm{H}\right\}$ NMR

$\mathrm{CDCl}_{3} 100 \mathrm{MHz}$

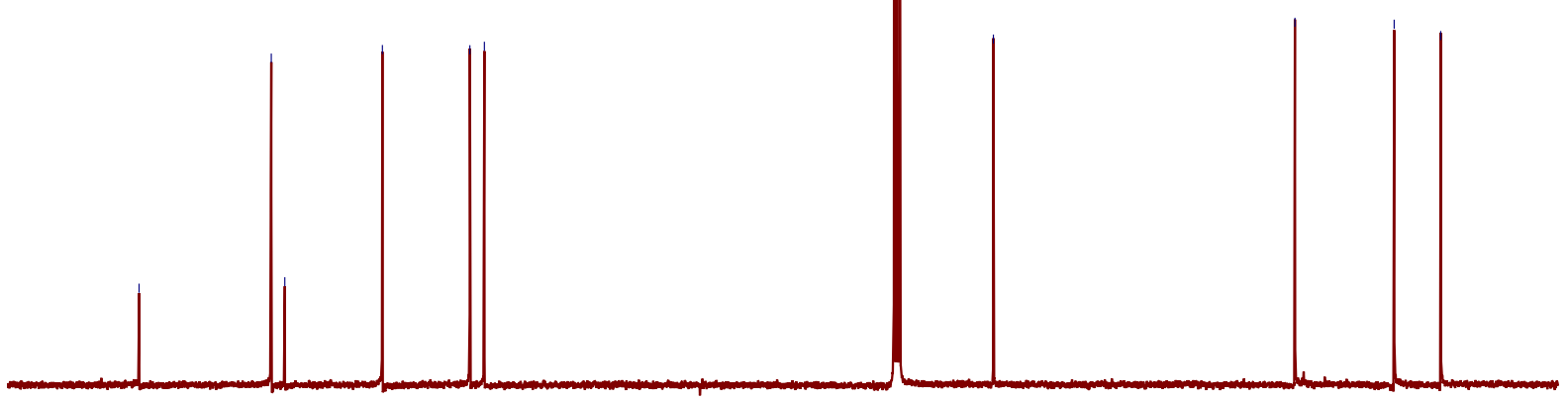

$80 \quad 170$

$\begin{array}{llllllll}60 & 150 & 140 & 130 & 120 & 110 & 100 & \begin{array}{c}90 \\ \mathrm{f} 1(\mathrm{ppm})\end{array}\end{array}$

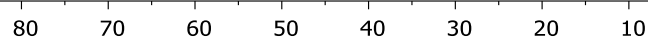

0 


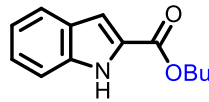

3md, ${ }^{1} \mathrm{H}$ NMR

$\mathrm{CDCl}_{3} 400 \mathrm{MHz}$

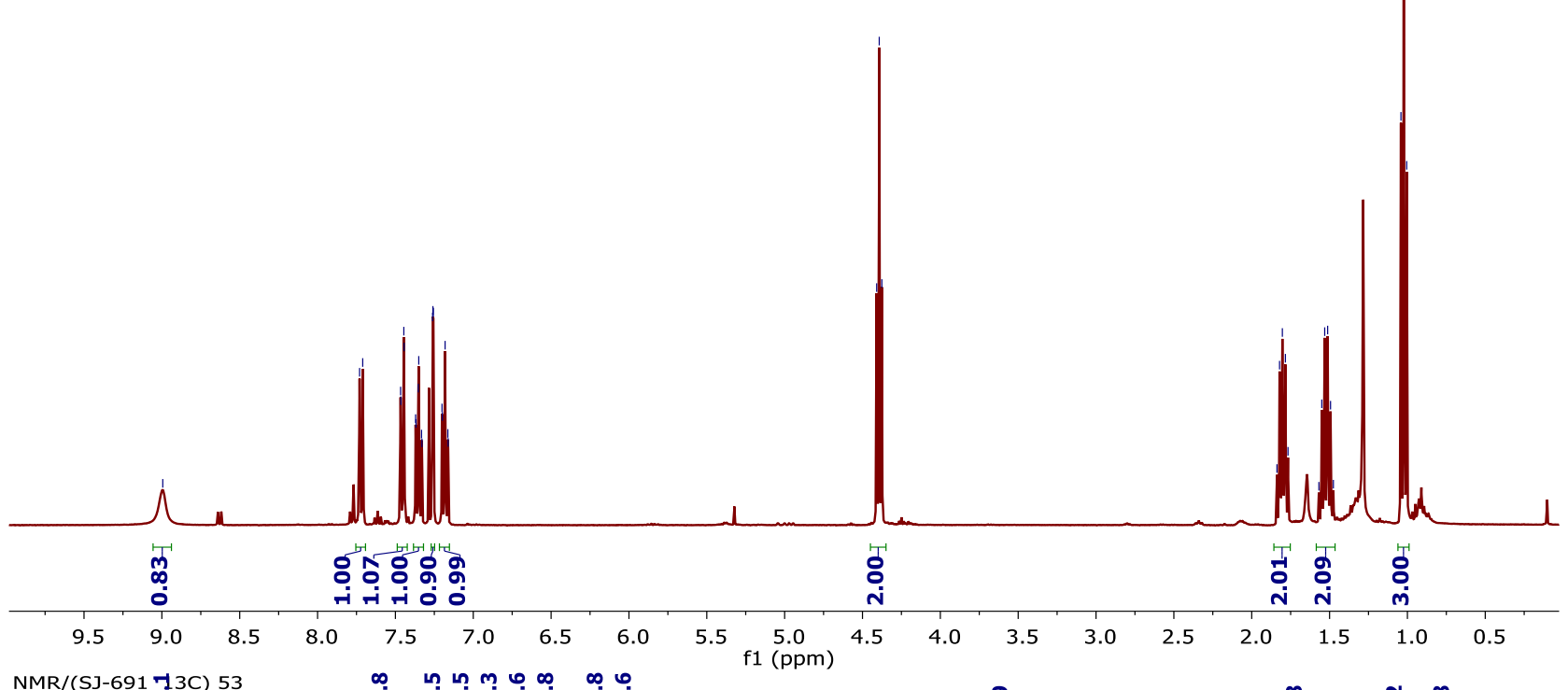
SJ-691(IN-2) ริ

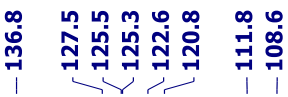
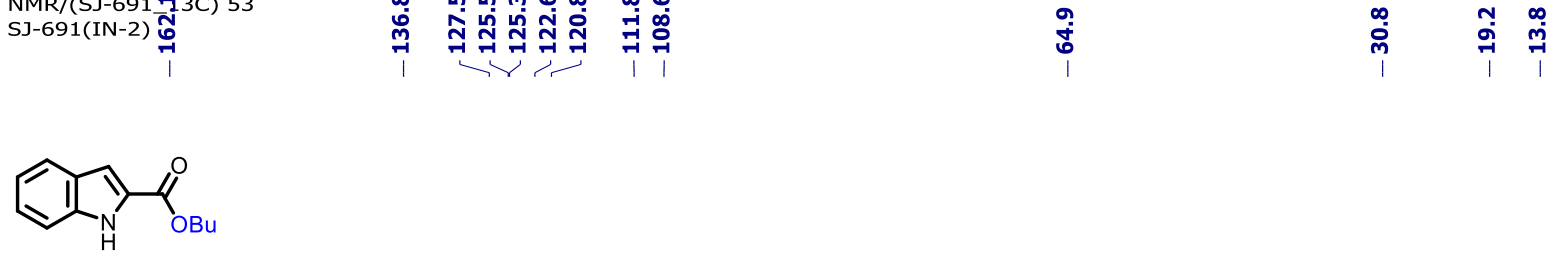

3md, ${ }^{13} \mathrm{C}\left\{{ }^{1} \mathrm{H}\right\}$ NMR $\mathrm{CDCl}_{3} 100 \mathrm{MHz}$

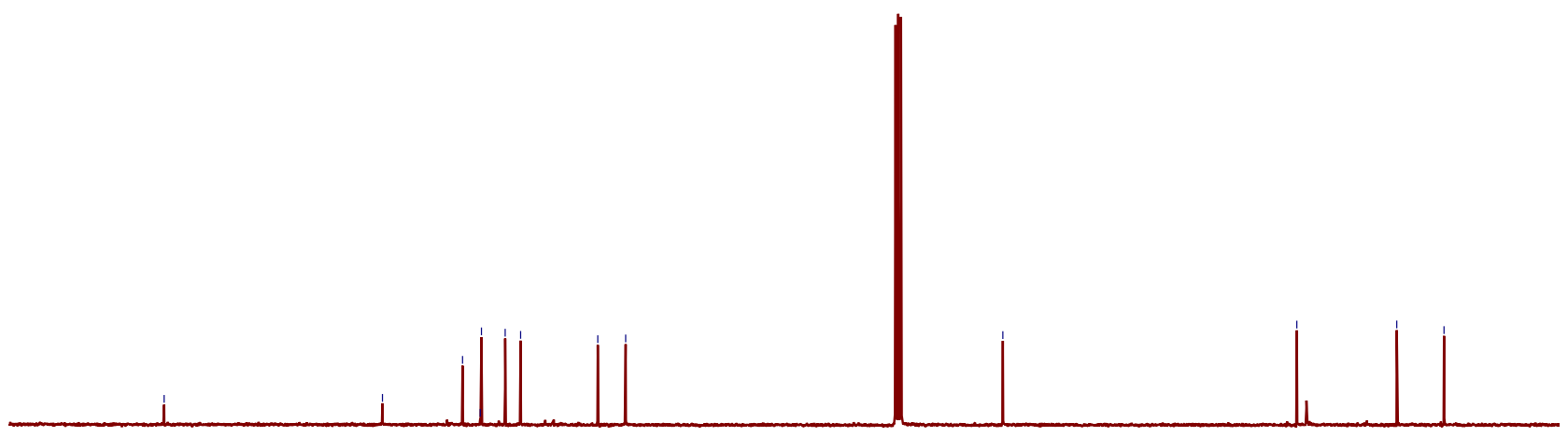

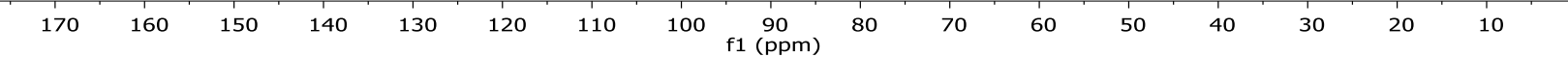




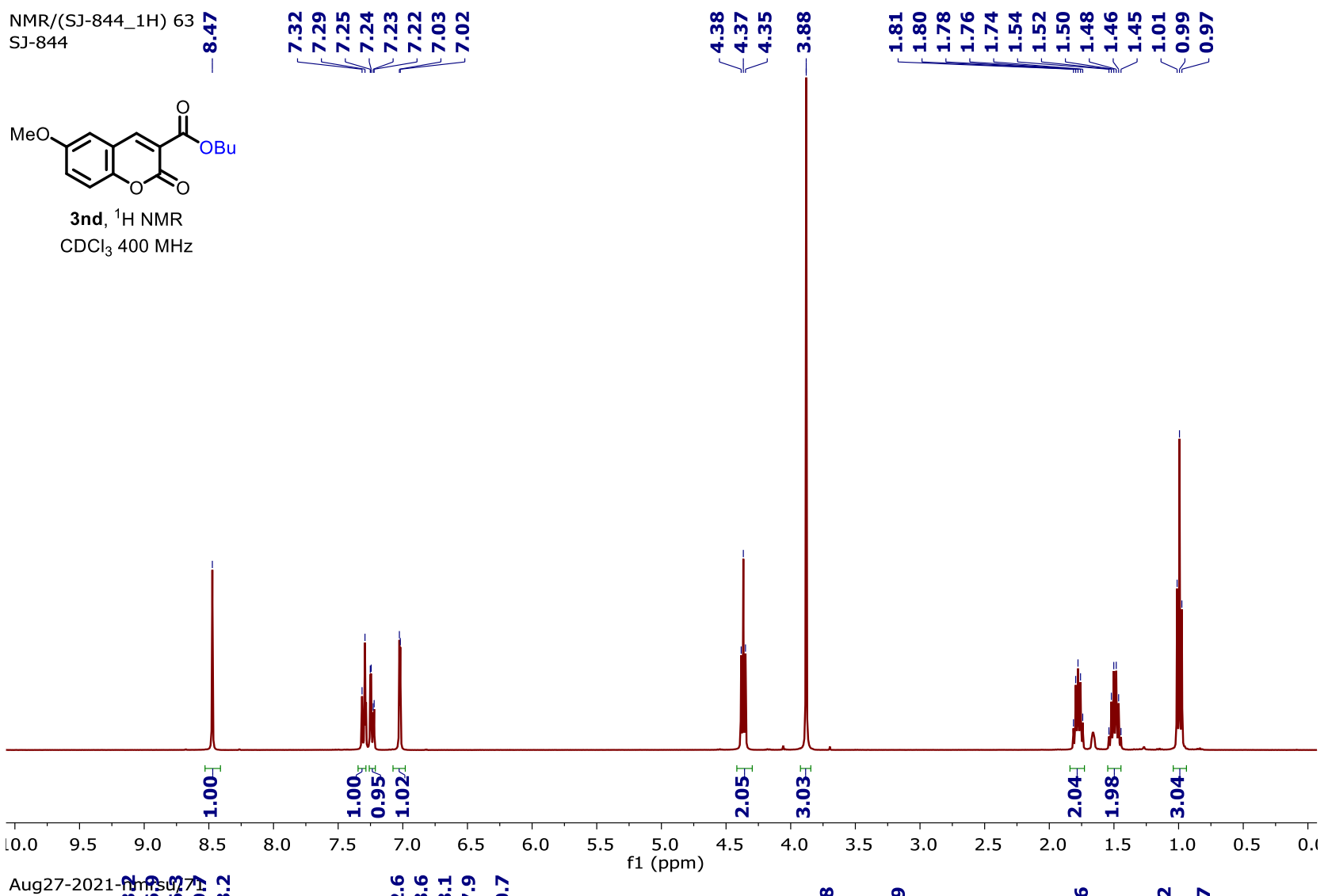

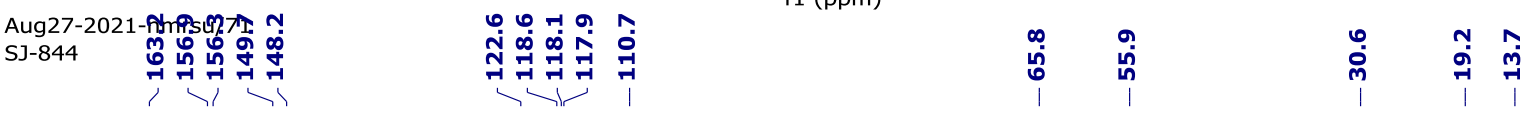

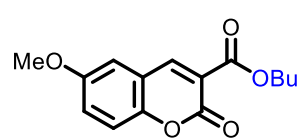

3nd, ${ }^{13} \mathrm{C}\left\{{ }^{1} \mathrm{H}\right\}$ NMR

$\mathrm{CDCl}_{3} 100 \mathrm{MHz}$

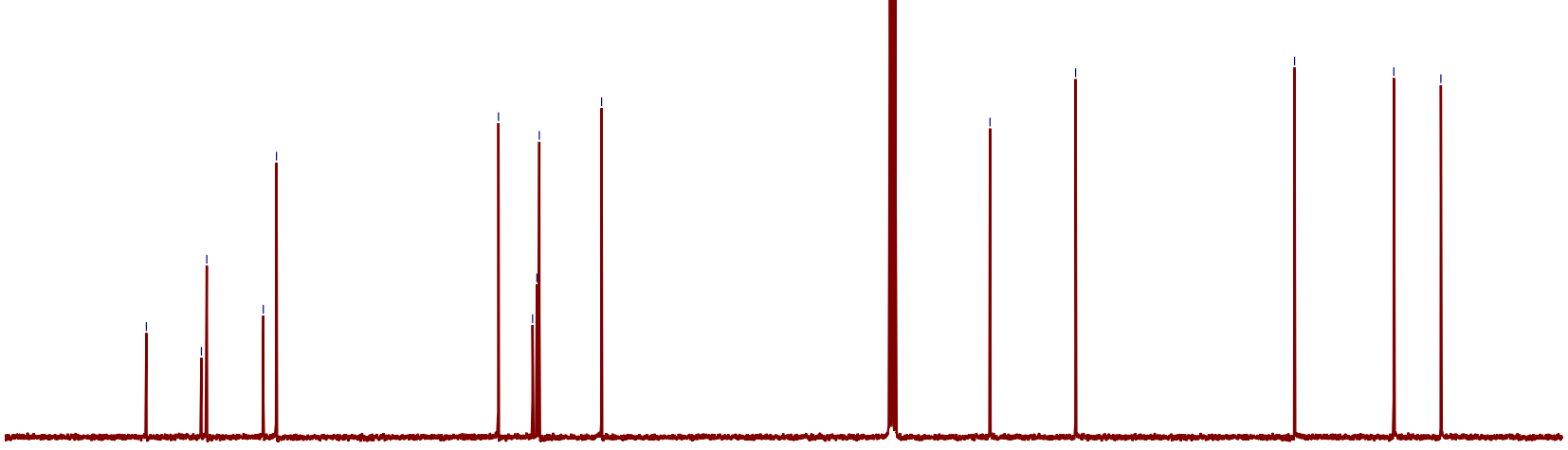

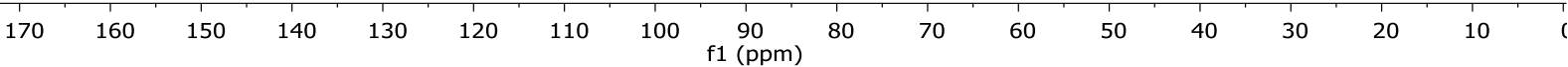




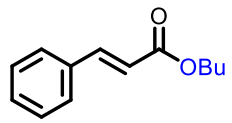

3od, ${ }^{1} \mathrm{H}$ NMR

$\mathrm{CDCl}_{3} 400 \mathrm{MHz}$

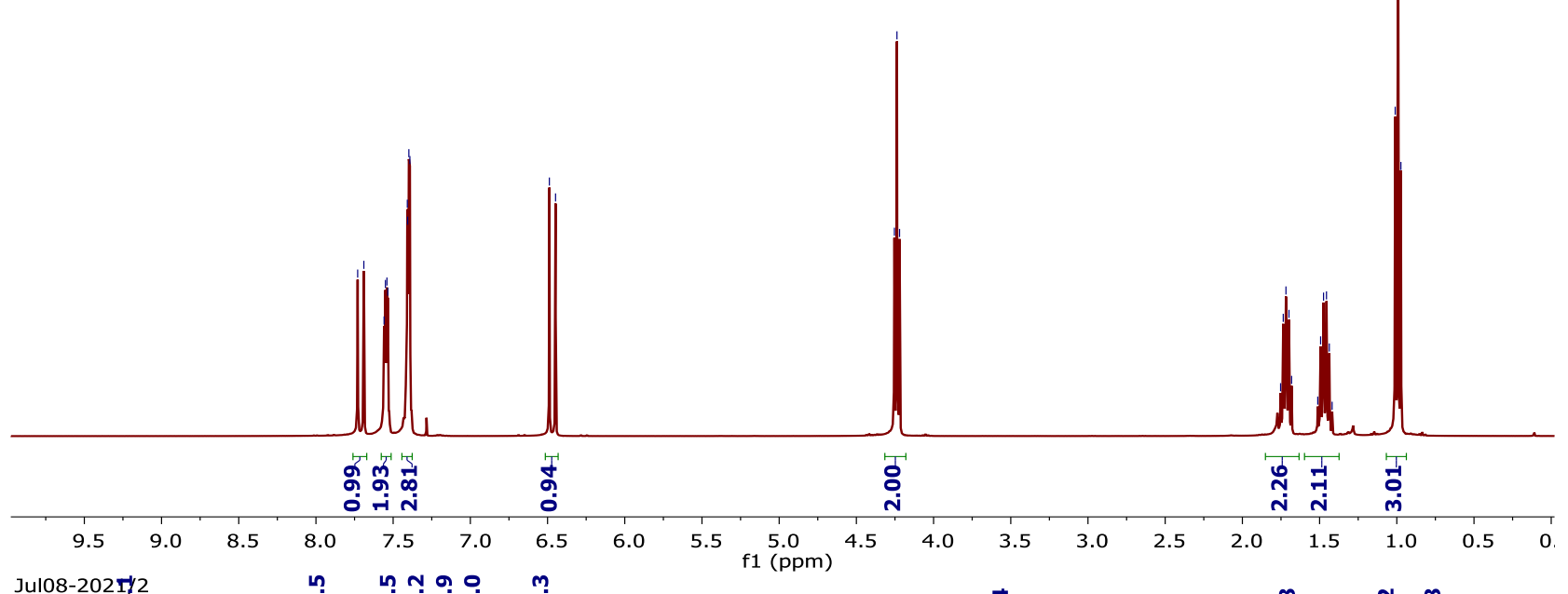

Jul08-202

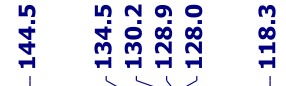

守

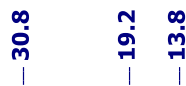

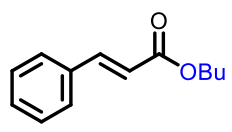

3od, ${ }^{13} \mathrm{C}\left\{{ }^{1} \mathrm{H}\right\}$ NMR

$\mathrm{CDCl}_{3} 100 \mathrm{MHz}$
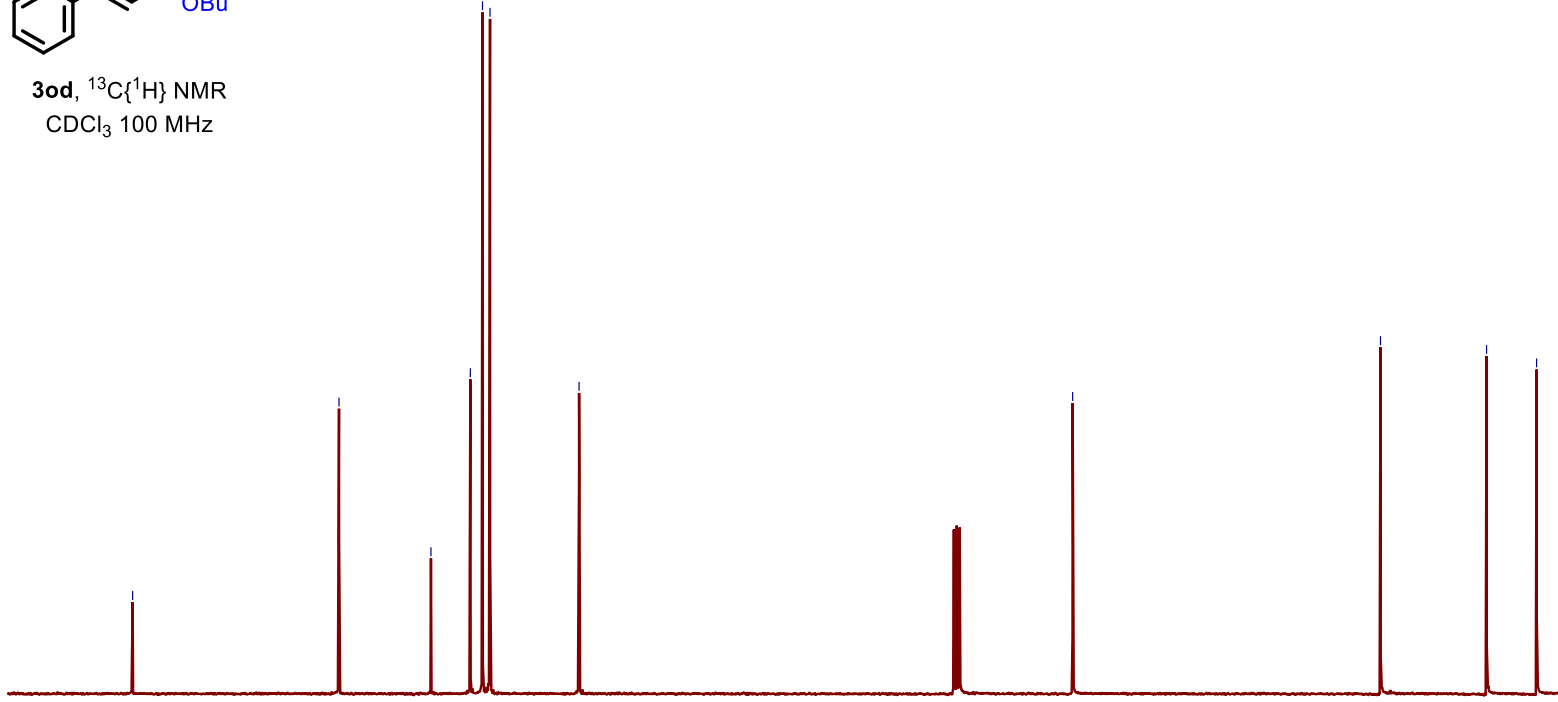

80
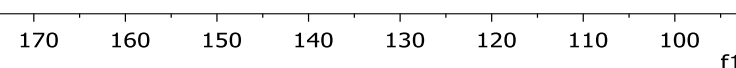

90
f1 (ppm)

80
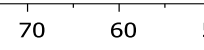

40

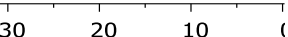




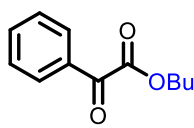

3pd, ${ }^{1} \mathrm{H}$ NMR

$\mathrm{CDCl}_{3} 400 \mathrm{MHz}$
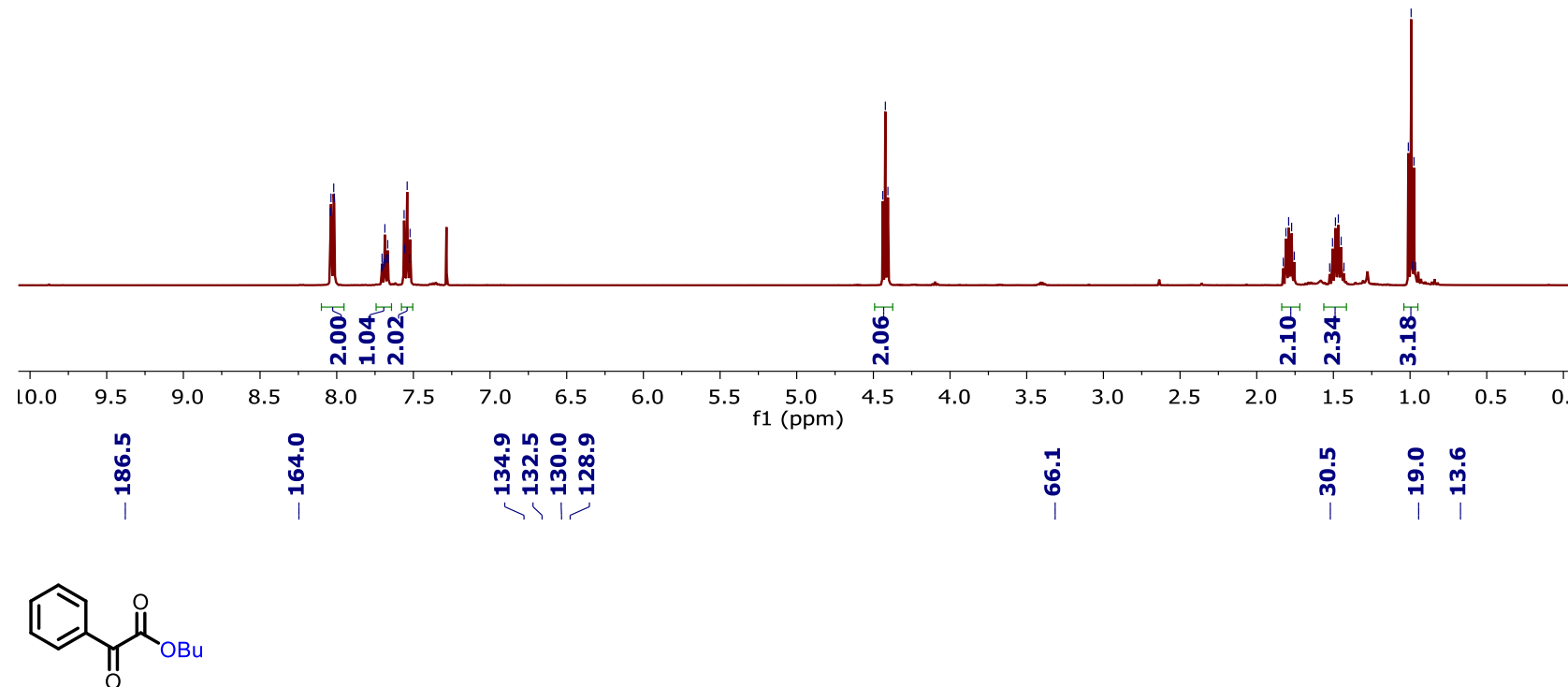

3pd, ${ }^{13} \mathrm{C}\left\{{ }^{1} \mathrm{H}\right\}$ NMR $\mathrm{CDCl}_{3} 100 \mathrm{MHz}$

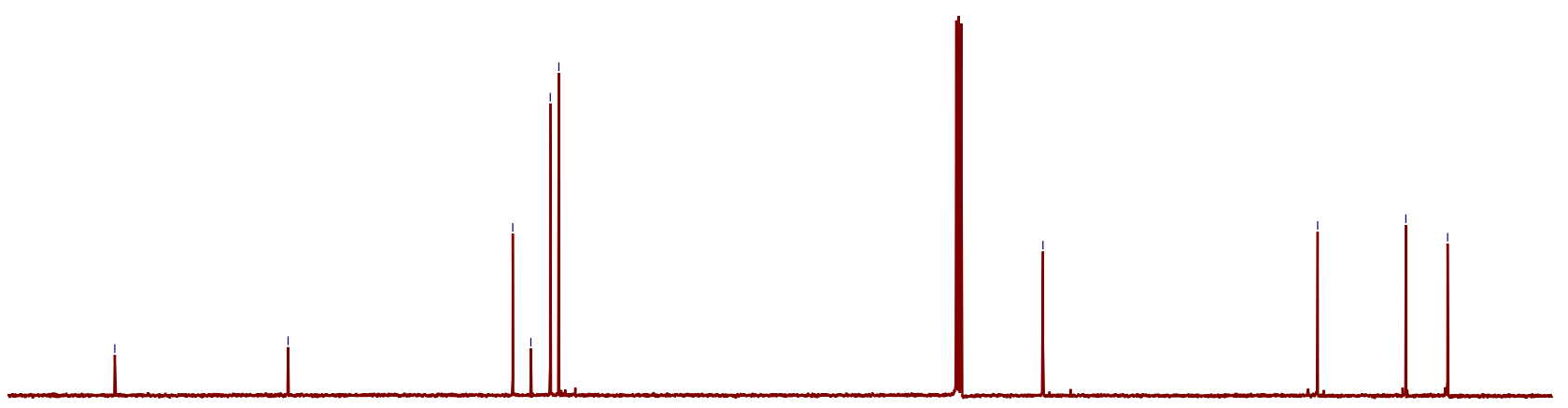

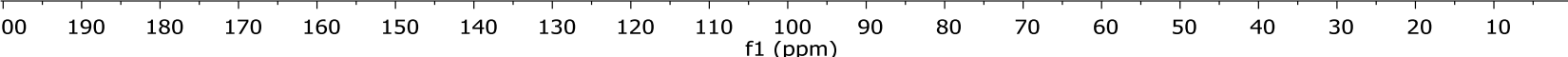


<smiles>[R]NCCCCCCC(=O)OCc1ccccc1</smiles>

3qd, ${ }^{1} \mathrm{H}$ NMR

$\mathrm{CDCl}_{3} 400 \mathrm{MHz}$
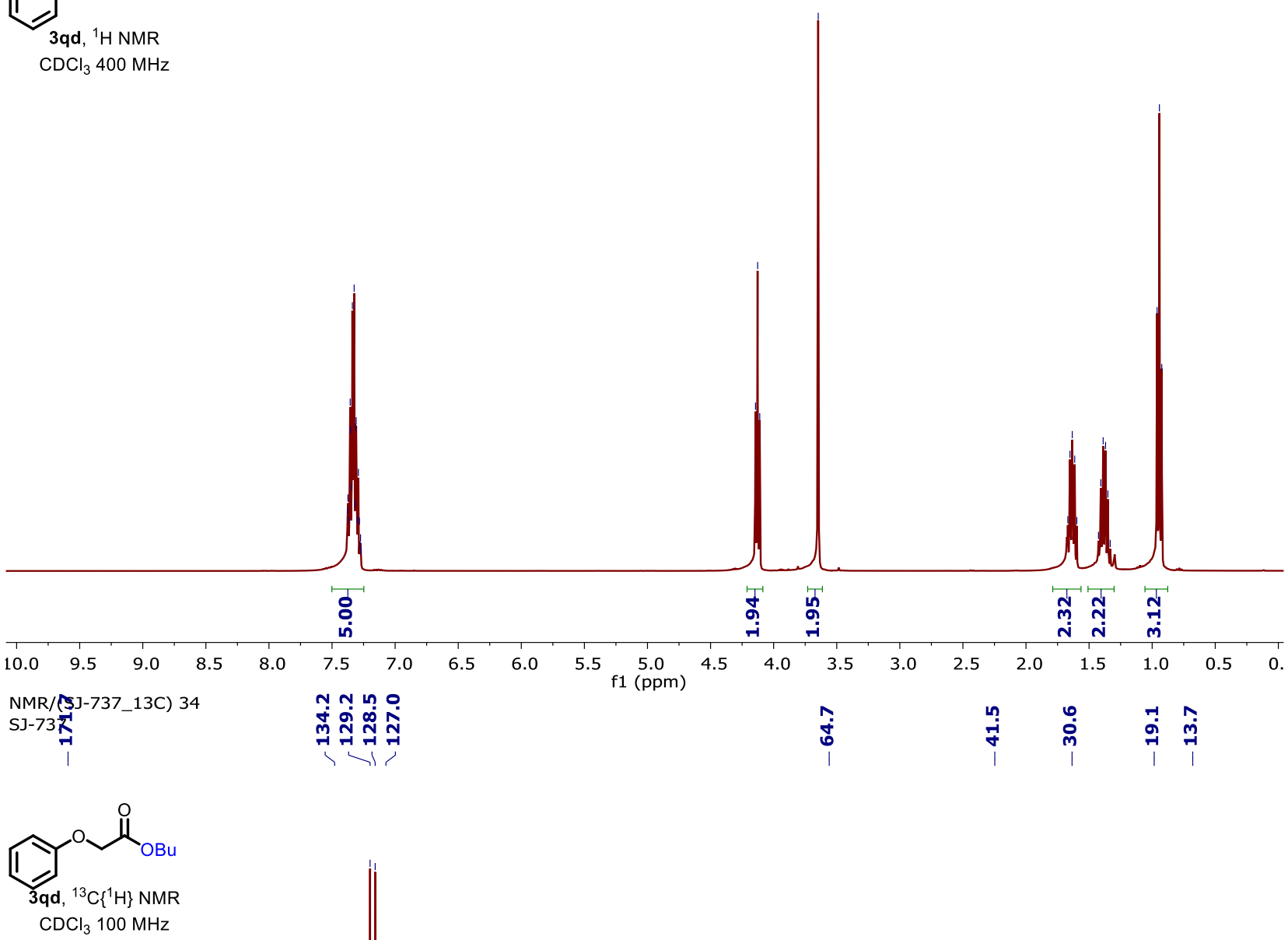

$\mathrm{CCl}_{3} 100 \mathrm{MHz}$
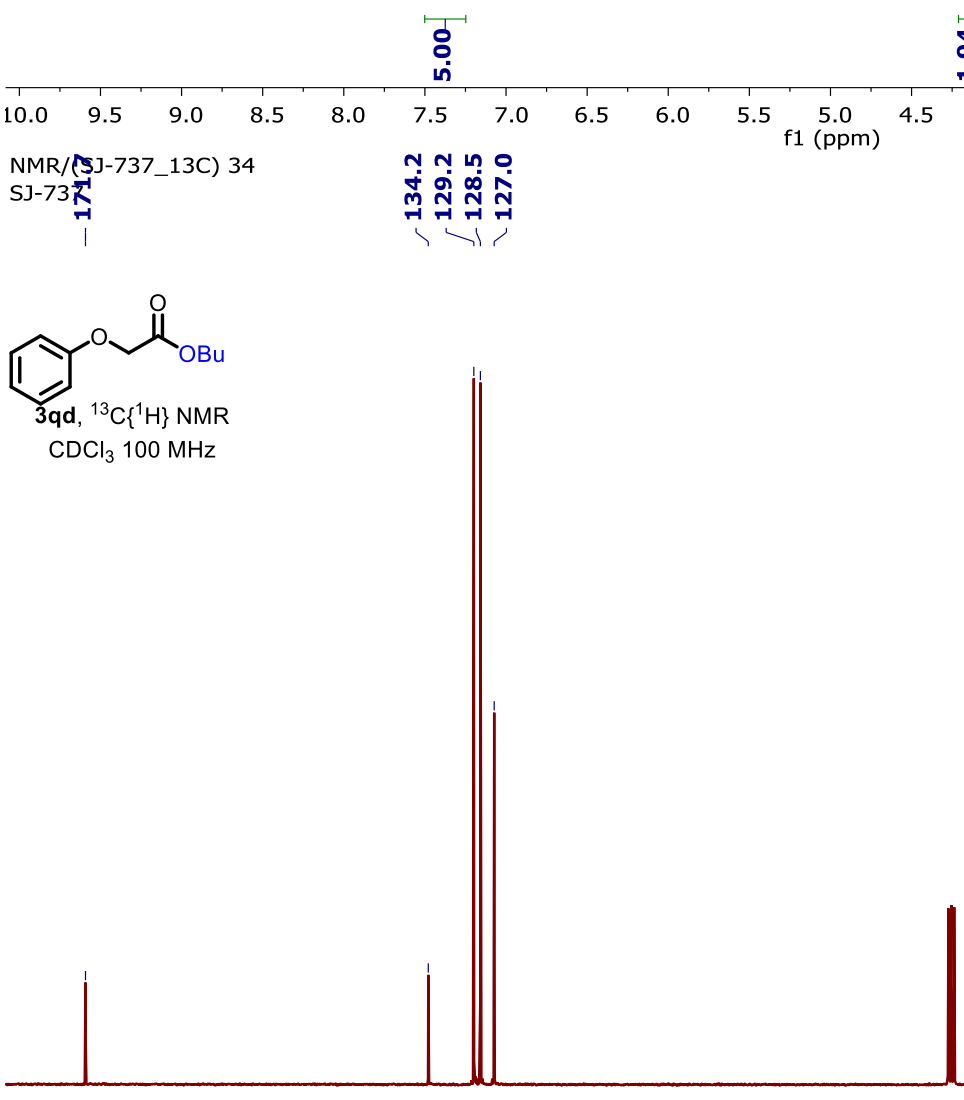

80
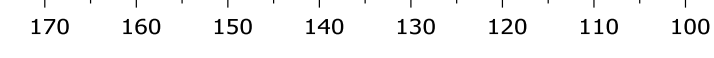

90
$\mathrm{f} 1(\mathrm{ppm})$

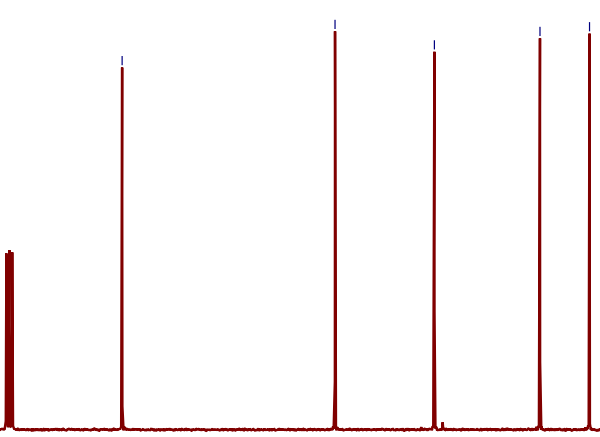


SJ-736

SJ-736

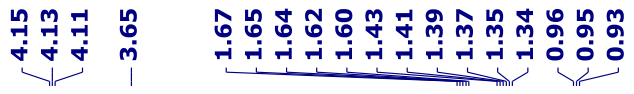

$1+\mathrm{H}_{\mathrm{OBu}}$

3rd, ${ }^{1} \mathrm{H}$ NMR

$\mathrm{CDCl}_{3} 400 \mathrm{MHz}$

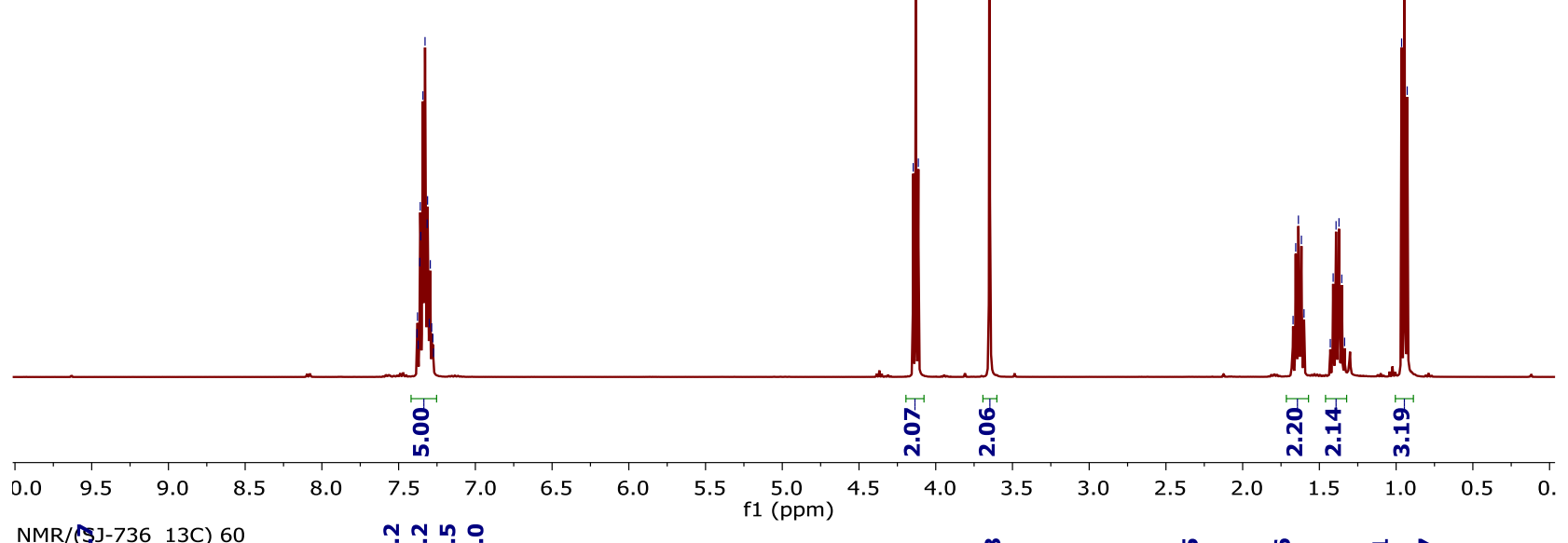

NMR/(\$J-736_13C) 60

SJ-73द्व

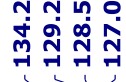

$\stackrel{\infty}{\text { g் }}$

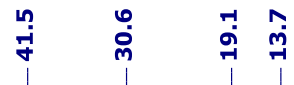

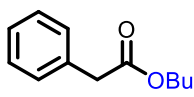

3rd, ${ }^{13} \mathrm{C}\left\{{ }^{1} \mathrm{H}\right\}$ NMR

$\mathrm{CDCl}_{3} 100 \mathrm{MHz}$
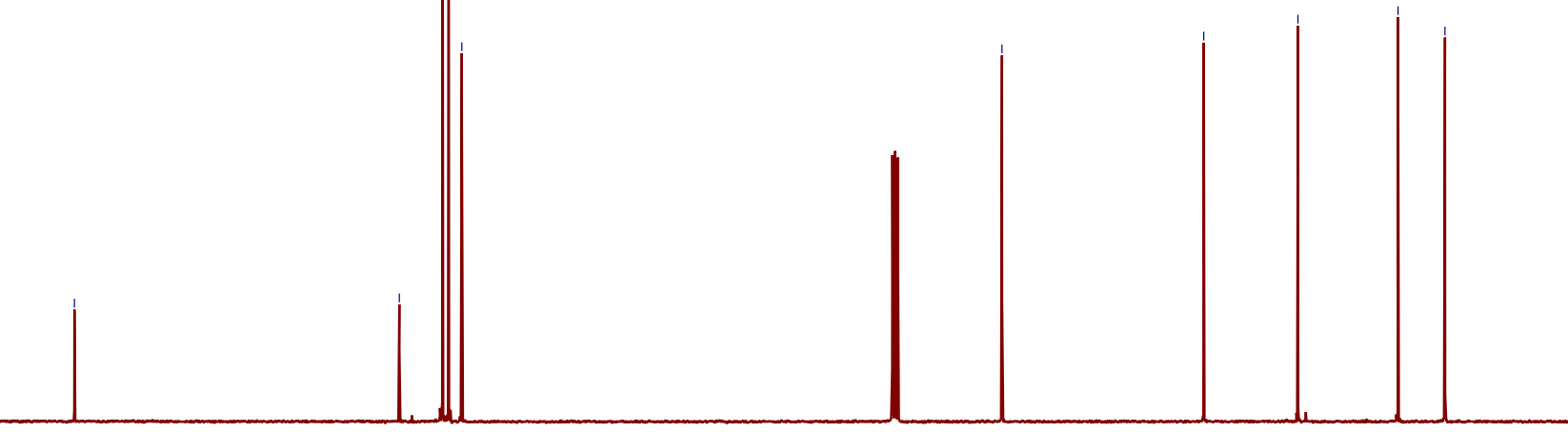

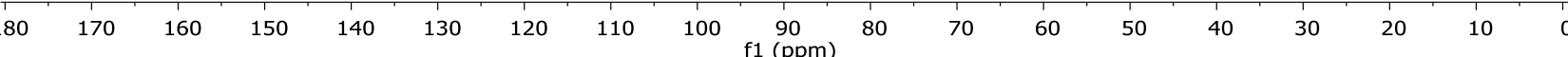


$\mathrm{CH}_{3}\left(\mathrm{CH}_{2}\right)_{16} \mathrm{COOBu}$

3sd, ${ }^{1} \mathrm{H}$ NMR

$\mathrm{CDCl}_{3} 400 \mathrm{MHz}$

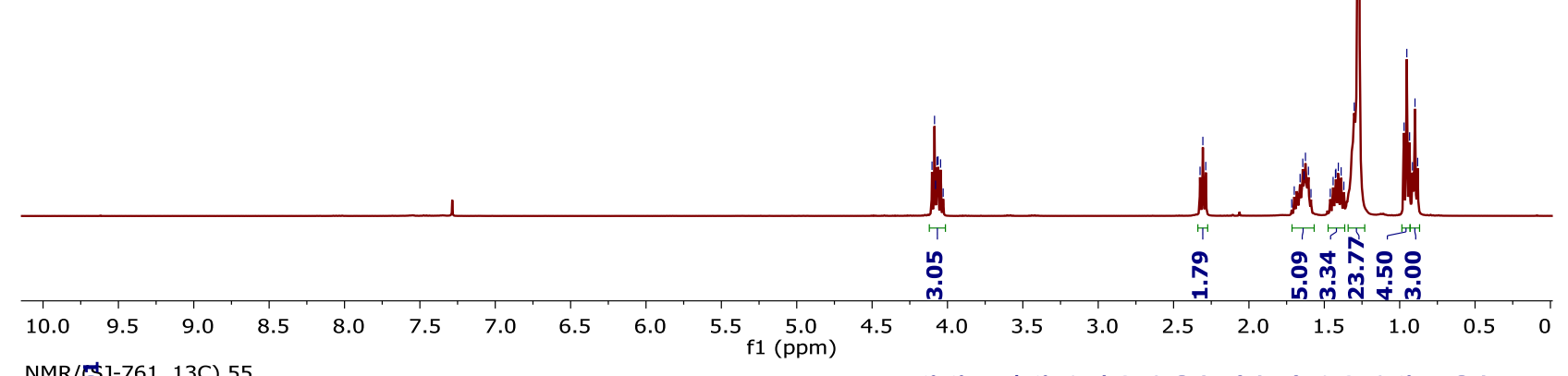

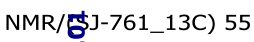
SJ-76椊

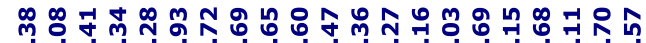
เ

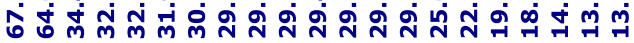

$\mathrm{CH}_{3}\left(\mathrm{CH}_{2}\right)_{16} \mathrm{COOBu}$

3sd, ${ }^{13} \mathrm{C}\left\{{ }^{1} \mathrm{H}\right\}$ NMR

$\mathrm{CDCl}_{3} 100 \mathrm{MHz}$

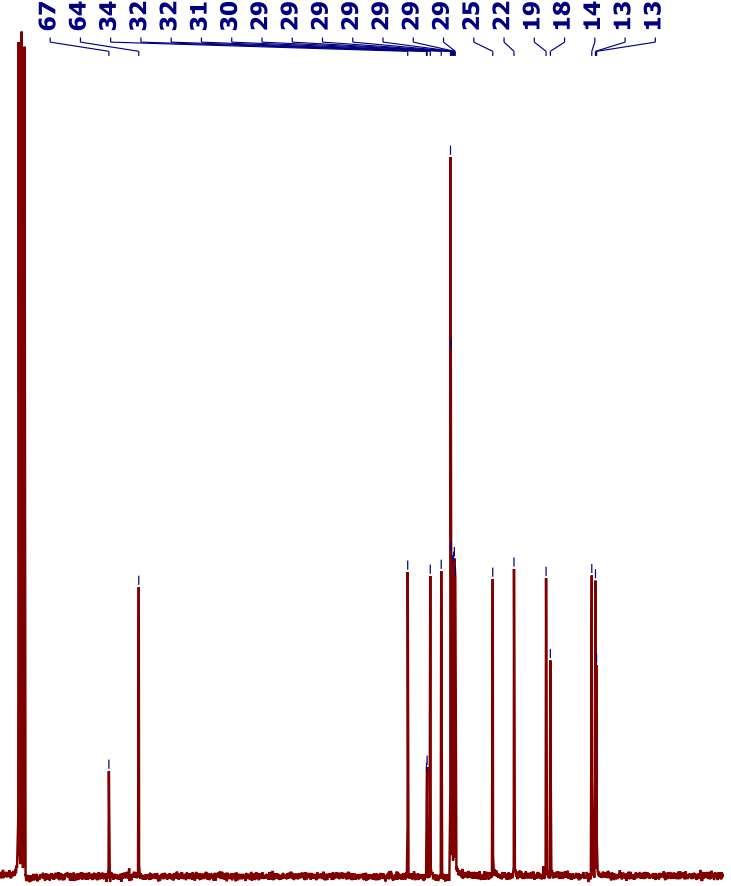

180

170160

160150

$150 \quad 140$

130

120

$110 \quad 100$

f1 (ppm)

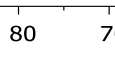

(2)


<smiles>CCCCOC(=O)CCC(=O)CCCC</smiles>

3td, ${ }^{1} \mathrm{H}$ NMR

$\mathrm{CDCl}_{3} 400 \mathrm{MHz}$

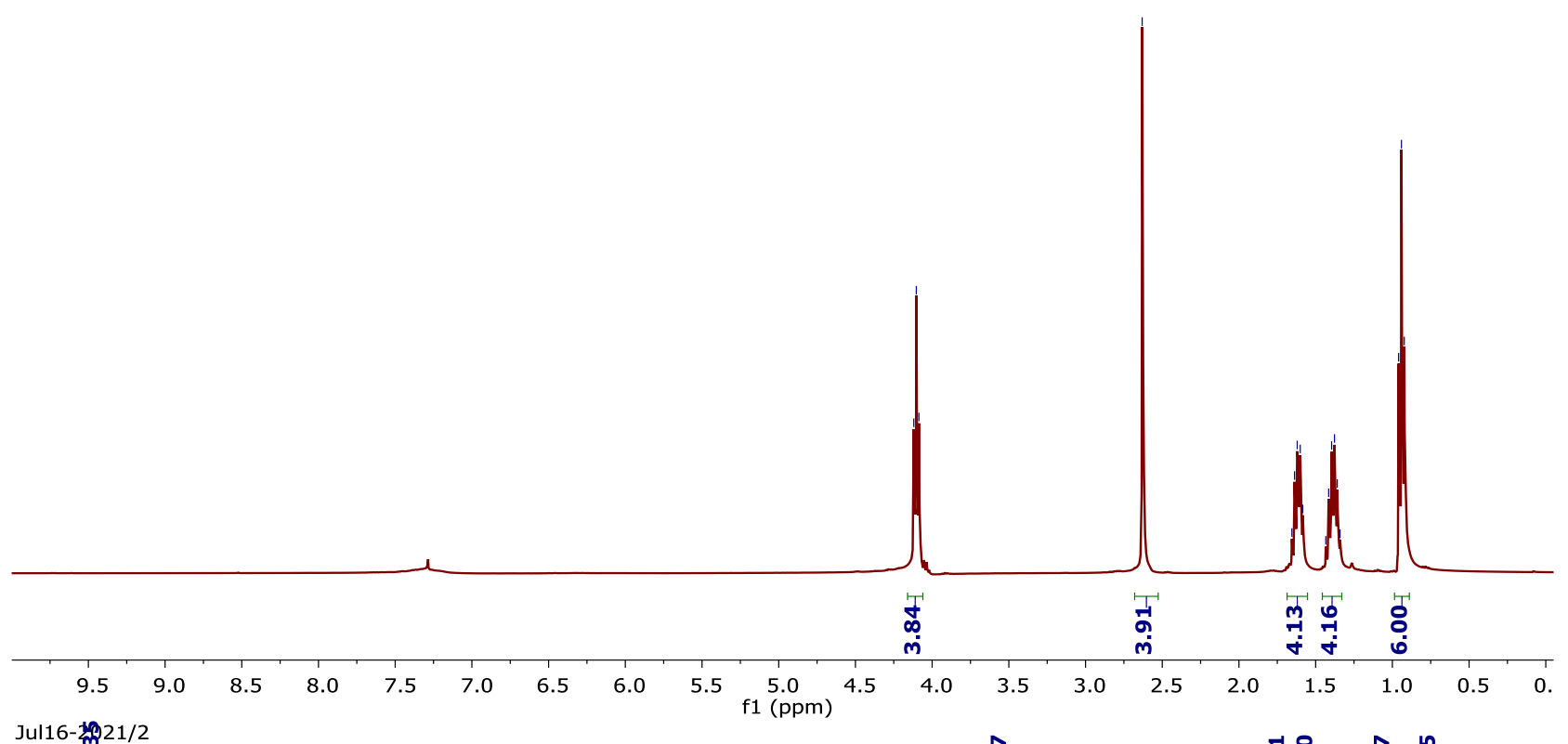

Jul16-慗 21/2

SJ-756 (5)

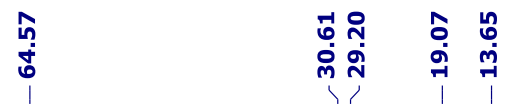<smiles>CCCCOC(=O)CCC(=O)OCCC</smiles>

3td, ${ }^{13} \mathrm{C}\left\{{ }^{1} \mathrm{H}\right\}$ NMR

$\mathrm{CDCl}_{3} 100 \mathrm{MHz}$

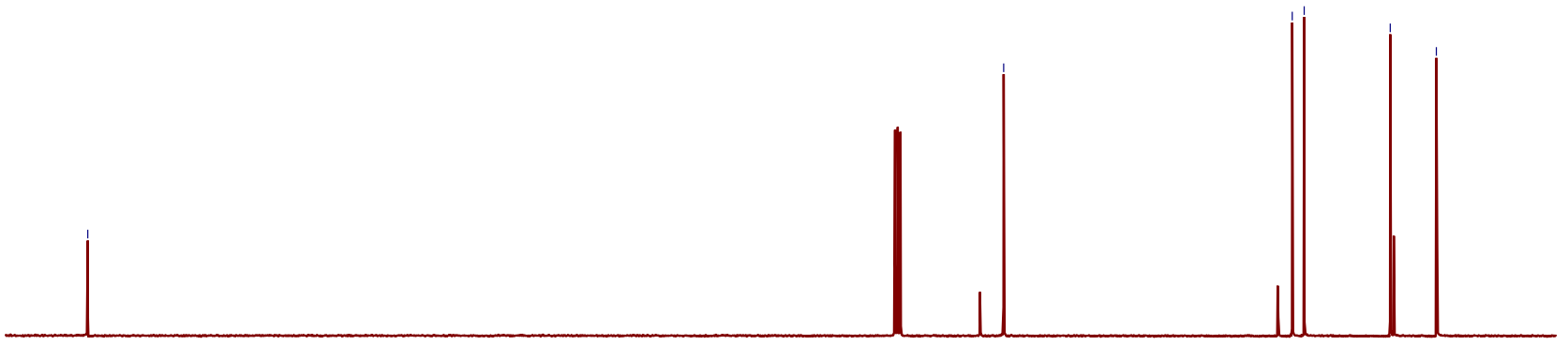

180

$170 \quad 160$

$150 \quad 140$

$110 \quad 100$

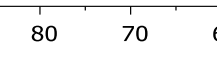

60 


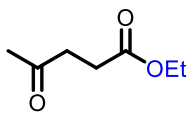

3ub, ${ }^{1} \mathrm{H}$ NMR

$\mathrm{CDCl}_{3} 400 \mathrm{MHz}$

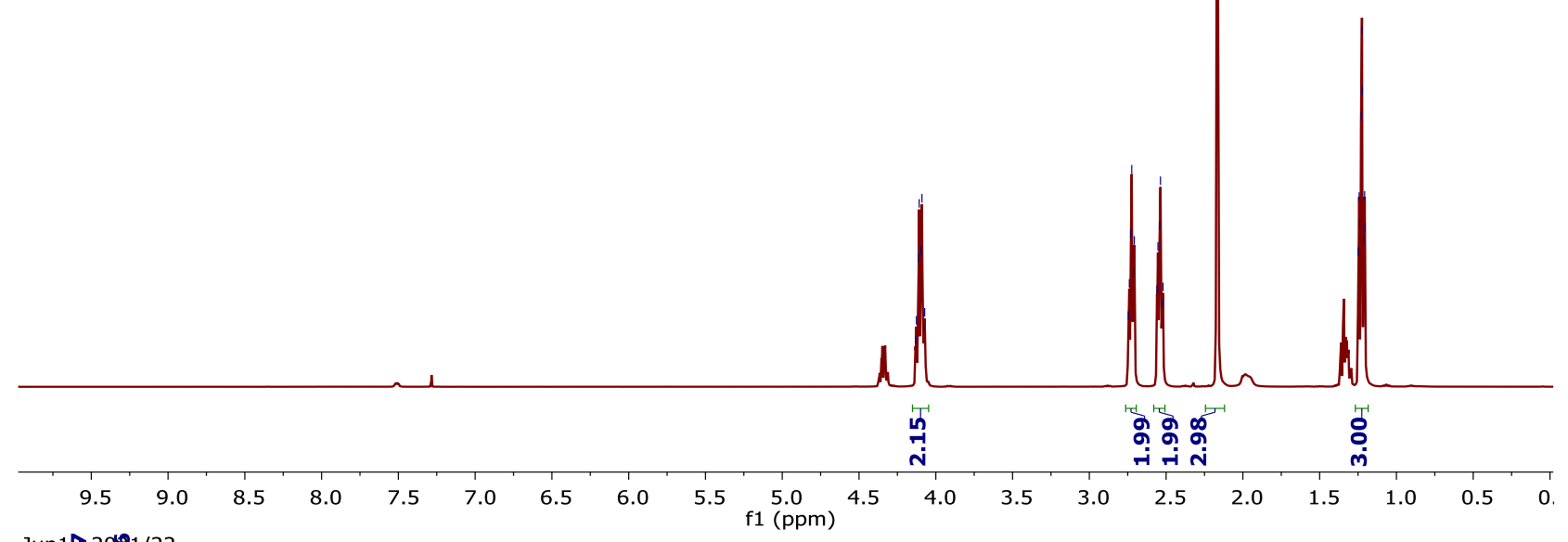
Jun18:-20\%1/22 SJ-8 6<smiles>CCOC(=O)CCC(C)=O</smiles>

3ub, ${ }^{13} \mathrm{C}\left\{{ }^{1} \mathrm{H}\right\}$ NMR $\mathrm{CDCl}_{3} 100 \mathrm{MHz}$
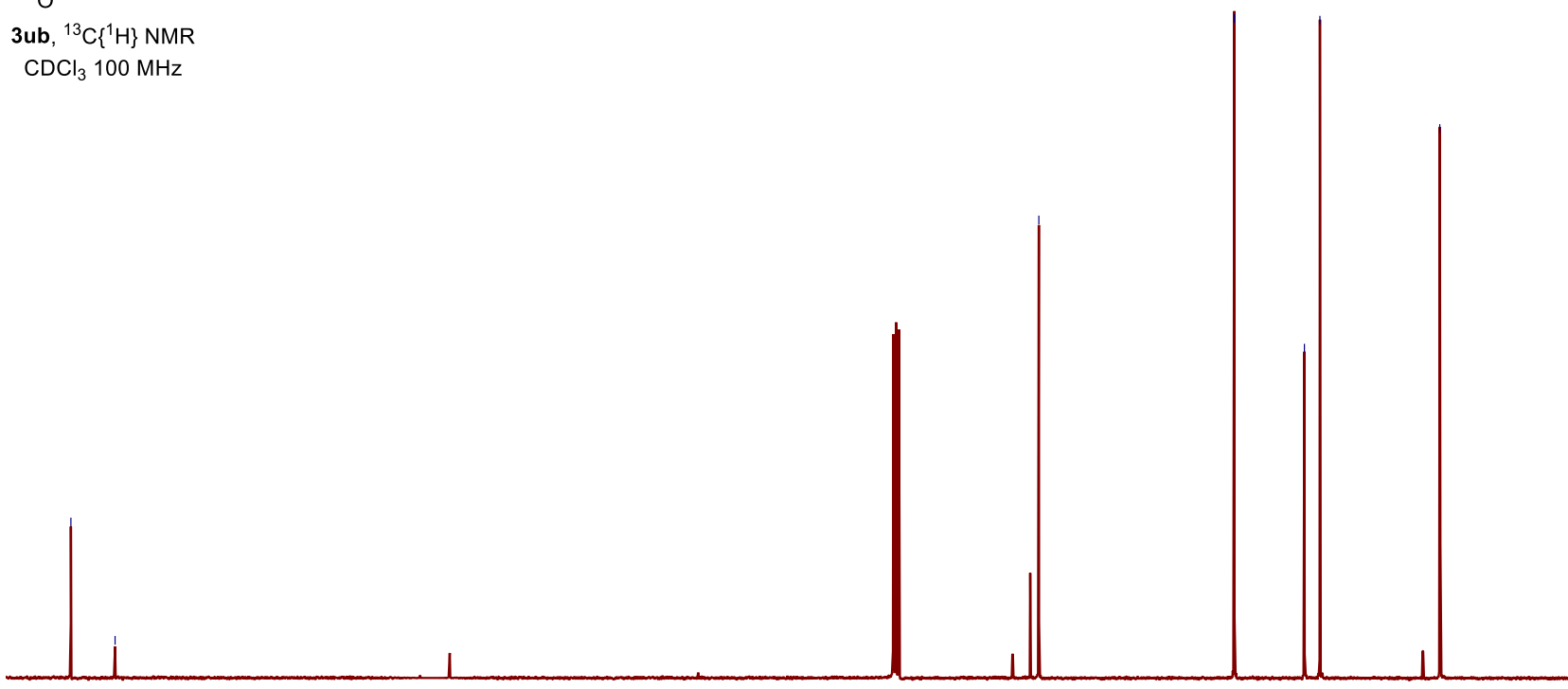

$80 \quad 70 \quad 60 \quad 50 \quad 40 \quad 30 \quad 30 \quad 10 \quad 0$


$\mathrm{H}_{3} \mathrm{C}\left(\mathrm{H}_{2} \mathrm{C}\right)_{4}=\left(\mathrm{CH}_{2}\right)_{7} \mathrm{COOBu}$

3vd, ${ }^{1} \mathrm{H}$ NMR

$\mathrm{CDCl}_{3} 400 \mathrm{MHz}$

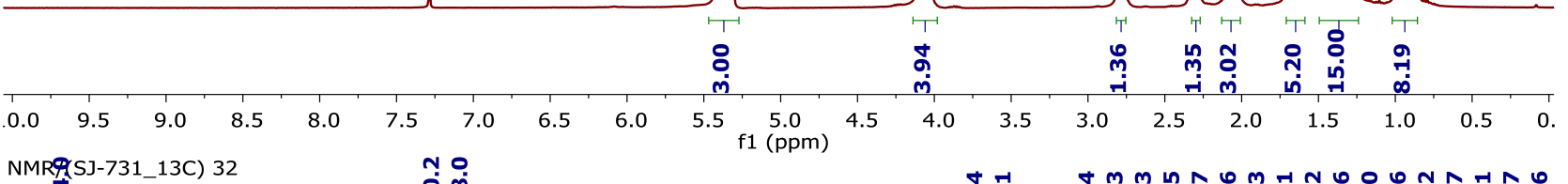

ํㅜㅇㅛ

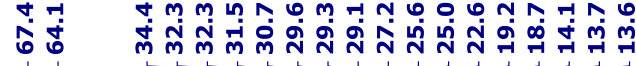

$\mathrm{H}_{3} \mathrm{C}\left(\mathrm{H}_{2} \mathrm{C}\right)_{4}=\left(\mathrm{CH}_{2}\right)_{7} \mathrm{COOBu}$

3vd, ${ }^{13} \mathrm{C}\left\{{ }^{1} \mathrm{H}\right\}$ NMR

$\mathrm{CDCl}_{3} 100 \mathrm{MHz}$

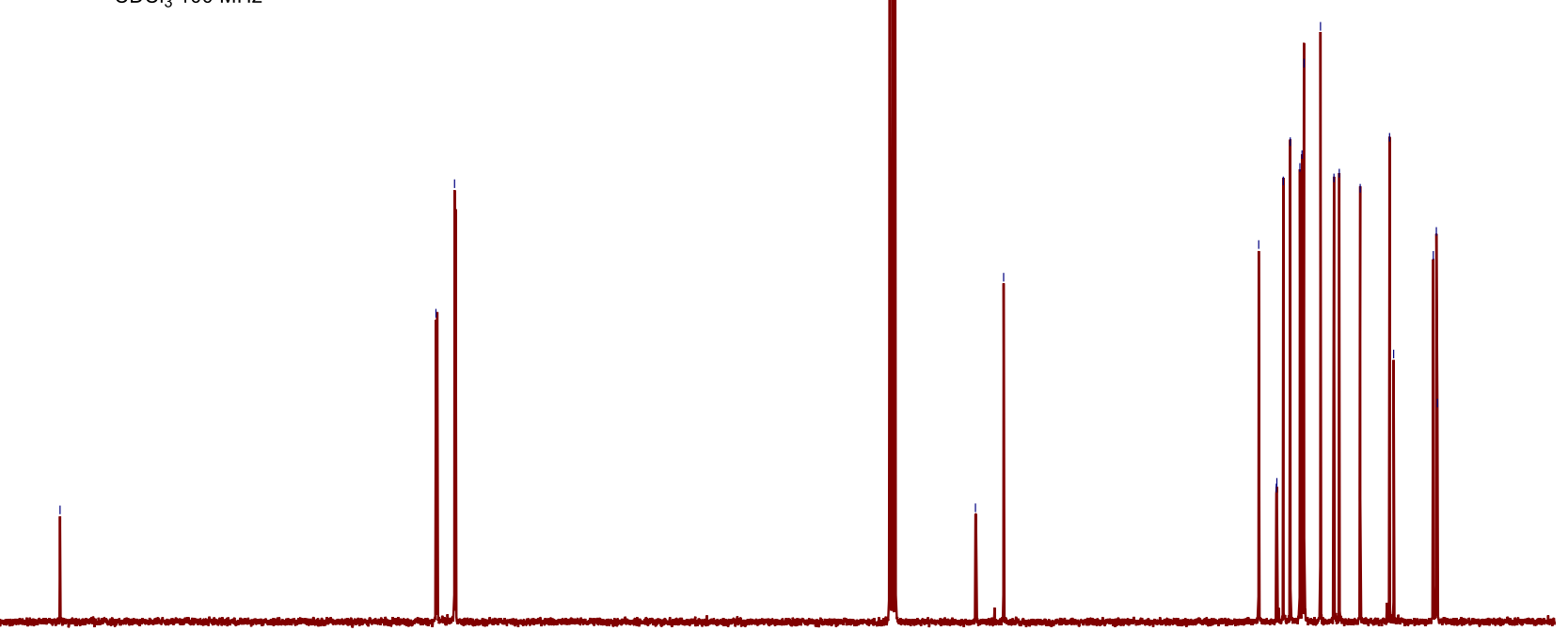

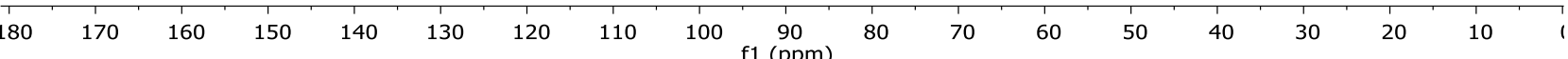


NMR/(SJ-757(eq)_1H) 31

$\mathrm{SJ}-757(\mathrm{eq})$
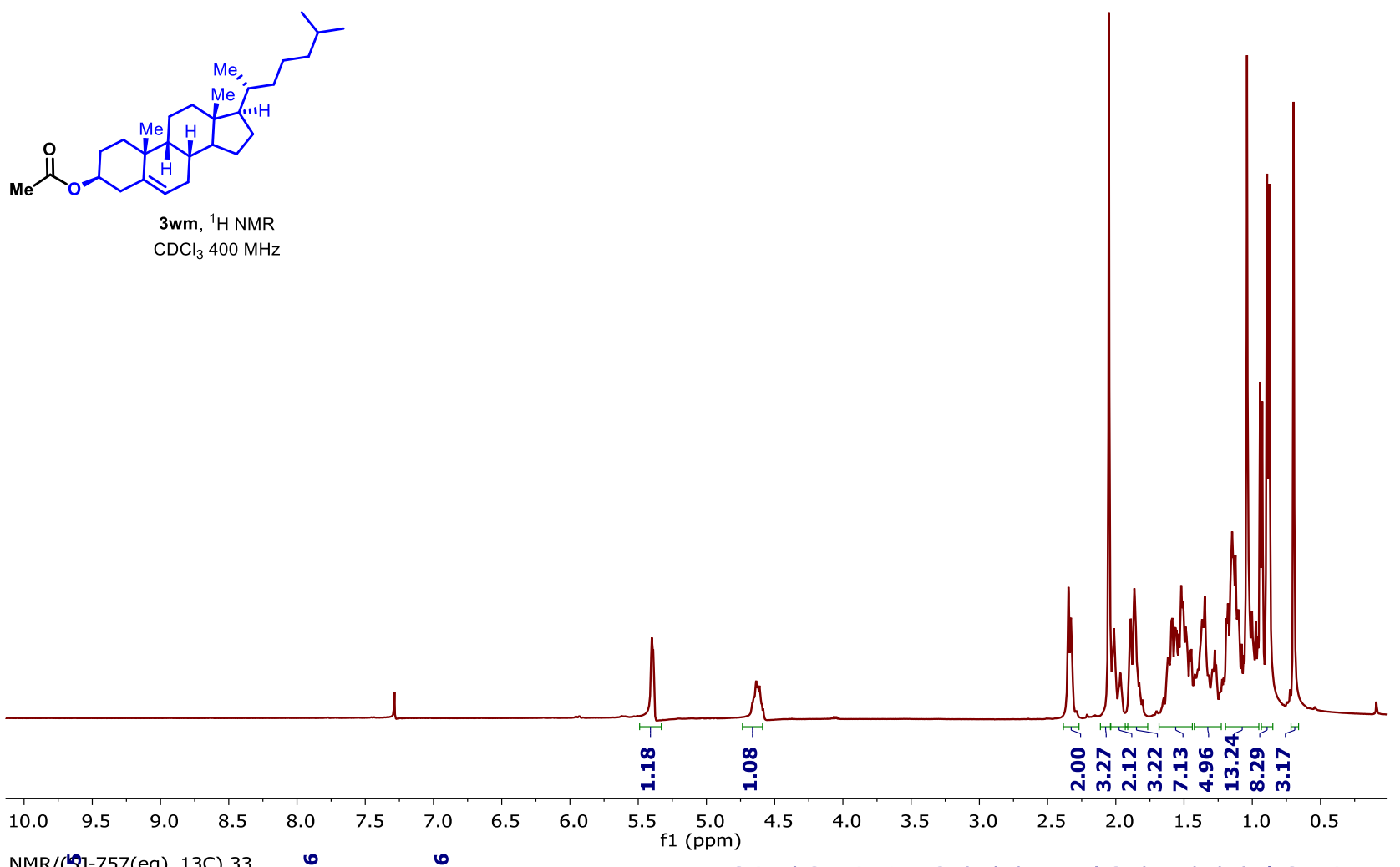

NMR/(20j-757(eq)_13C) 33 SJ-75些eq)

$\stackrel{0}{0} \underset{\stackrel{0}{m}}{\stackrel{N}{*}}$

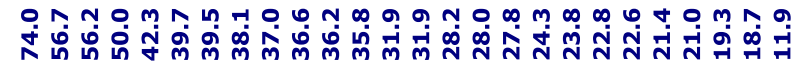

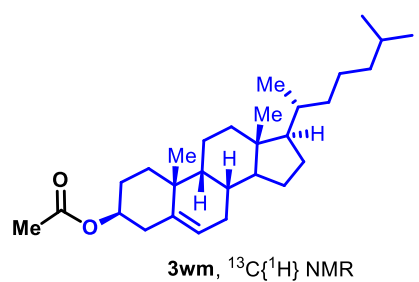

$\mathrm{CDCl}_{3} 100 \mathrm{MHz}$

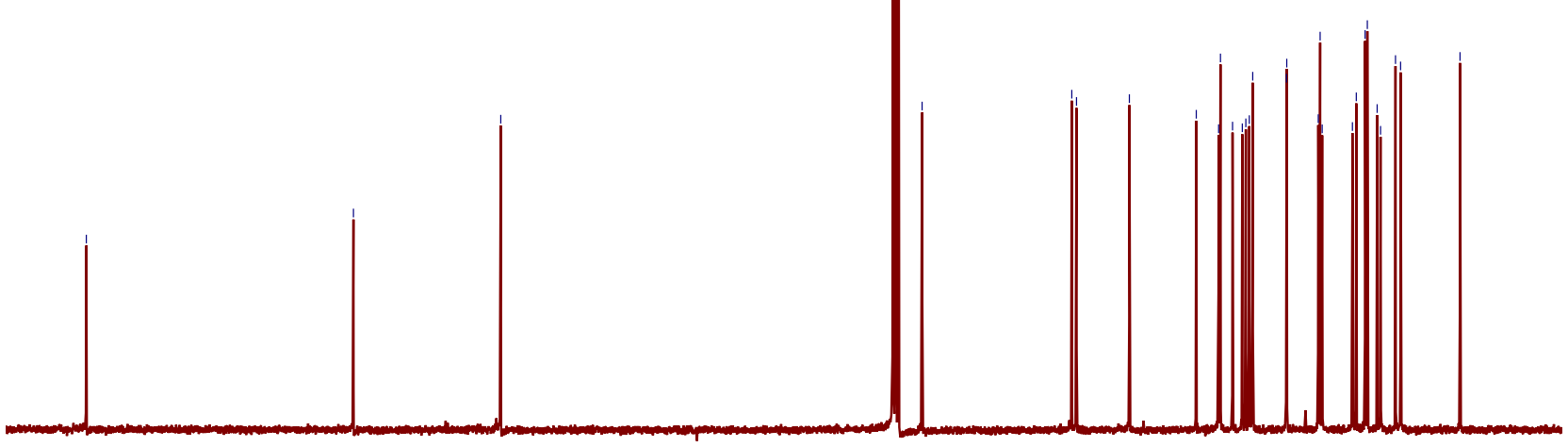

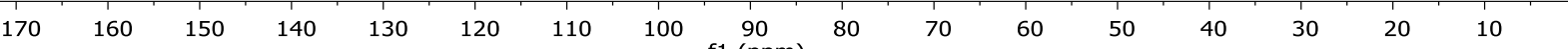




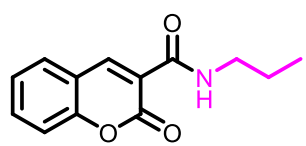

5aa, ${ }^{1} \mathrm{H}$ NMR $\mathrm{CDCl}_{3}, 400 \mathrm{MHz}$

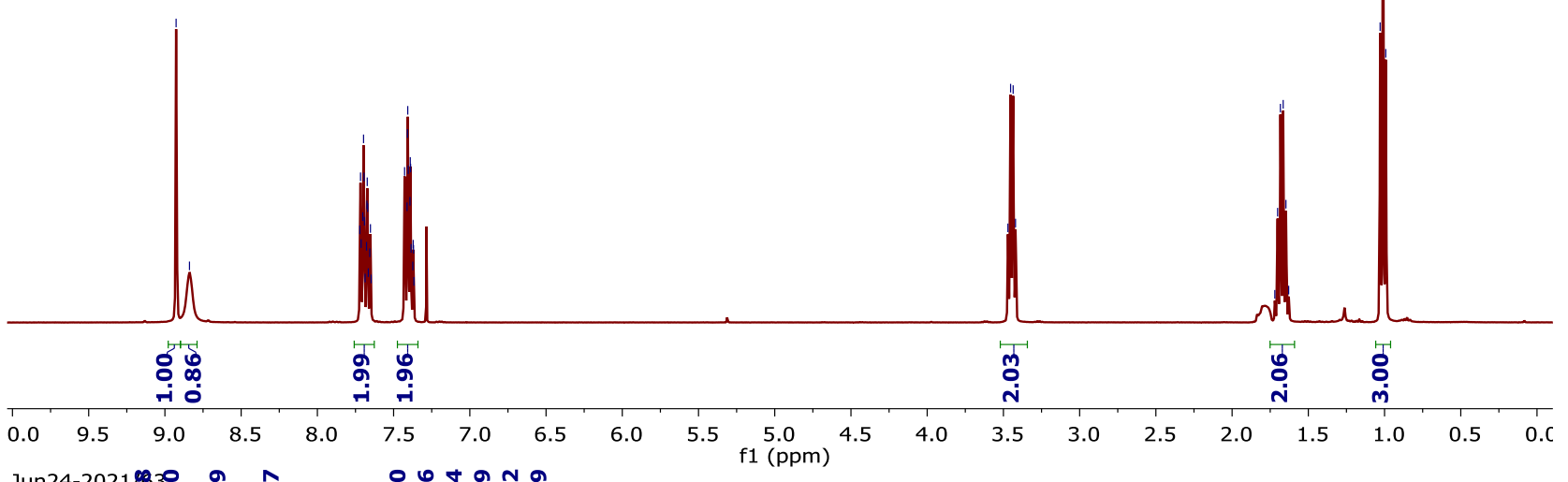

Jun24-2021

SJ-782(L)

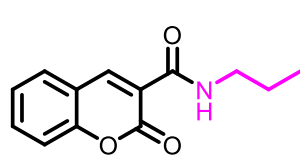

5aa, ${ }^{13} \mathrm{C}\left\{{ }^{1} \mathrm{H}\right\}$ NMR

$\mathrm{CDCl}_{3}, 100 \mathrm{MHz}$

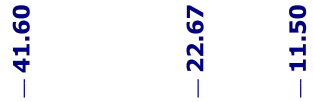

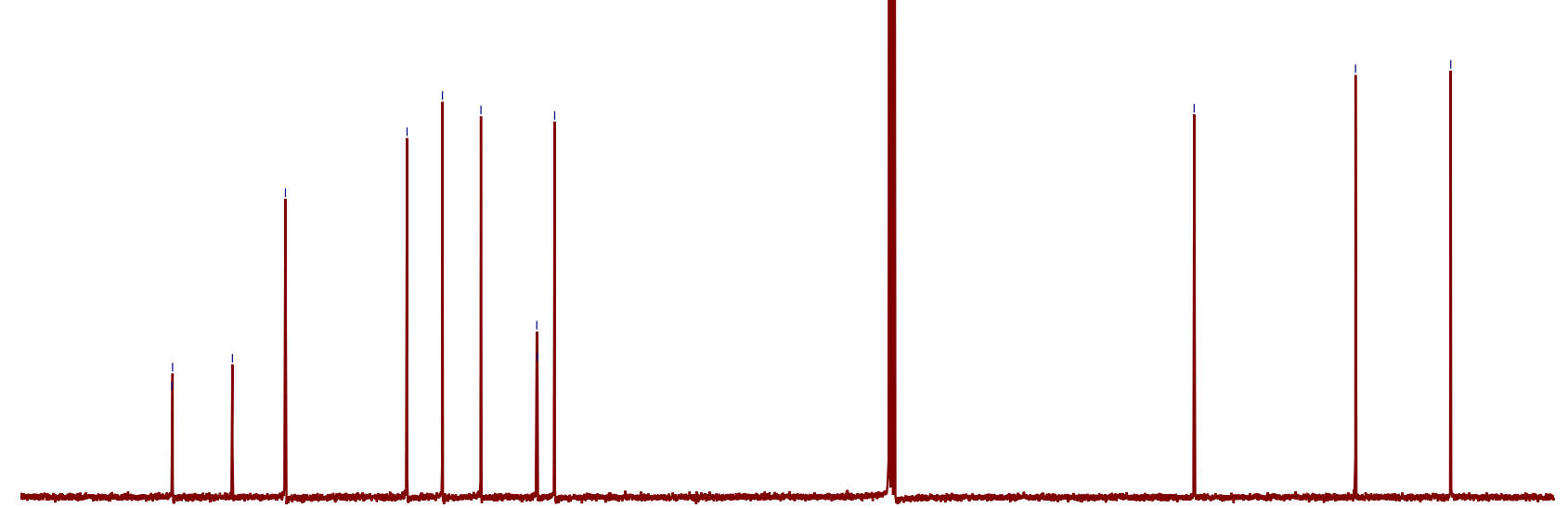

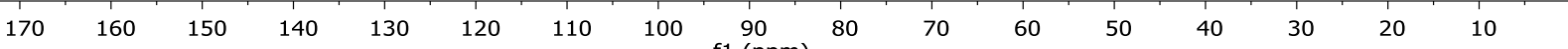




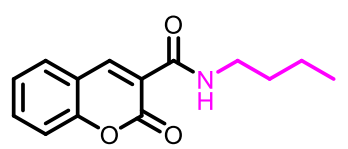

$5 \mathrm{ab},{ }^{1} \mathrm{H}$ NMR

$\mathrm{CDCl}_{3}, 400 \mathrm{MHz}$

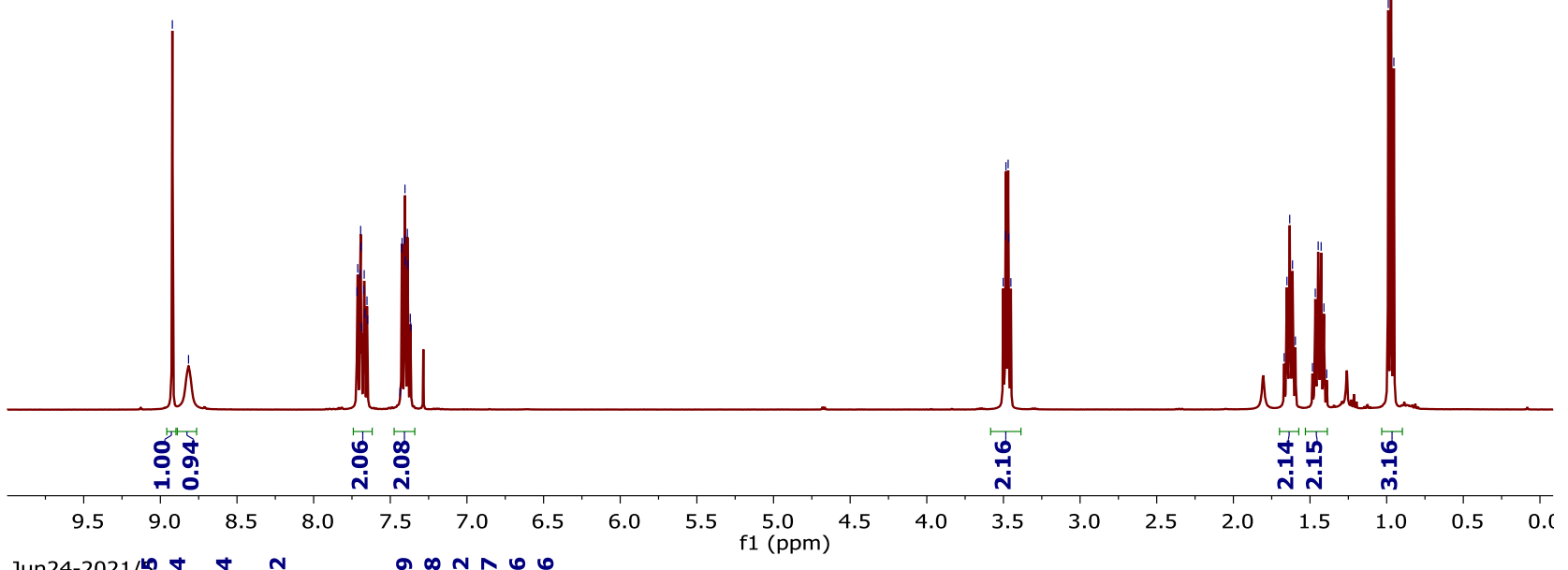

Jun24-2021/Sొ

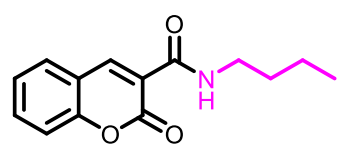

$5 \mathrm{ab},{ }^{13} \mathrm{C}\left\{{ }^{1} \mathrm{H}\right\}$ NMR

$\mathrm{CDCl}_{3}, 100 \mathrm{MHz}$

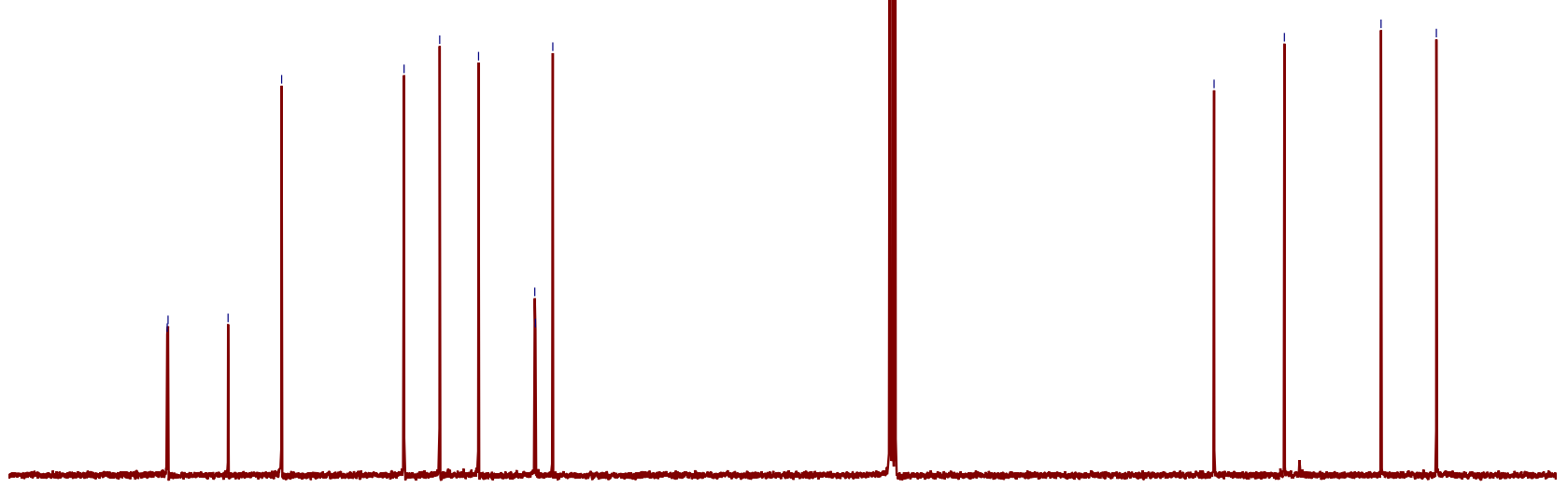

170

$160 \quad 150$

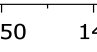

$140 \quad 130$
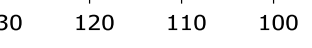

f1 $\stackrel{90}{(\mathrm{ppm})}$

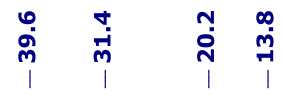


<smiles>O=C(NC1CCCCC1)c1cc2ccccc2oc1=O</smiles>

5ac, ${ }^{1} \mathrm{H}$ NMR

$\mathrm{CDCl}_{3}, 400 \mathrm{MHz}$

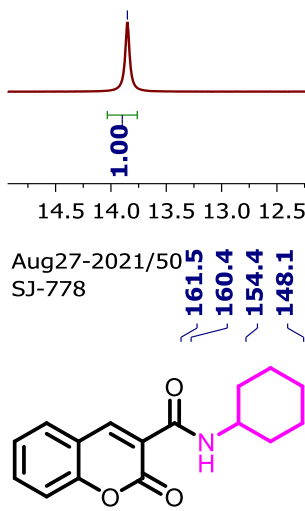

$5 \mathrm{ac},{ }^{13} \mathrm{C}\left\{{ }^{1} \mathrm{H}\right\}$ NMR

$\mathrm{CDCl}_{3}, 100 \mathrm{MHz}$

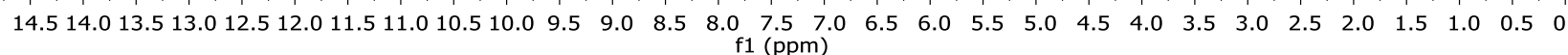

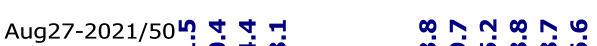

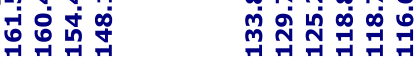
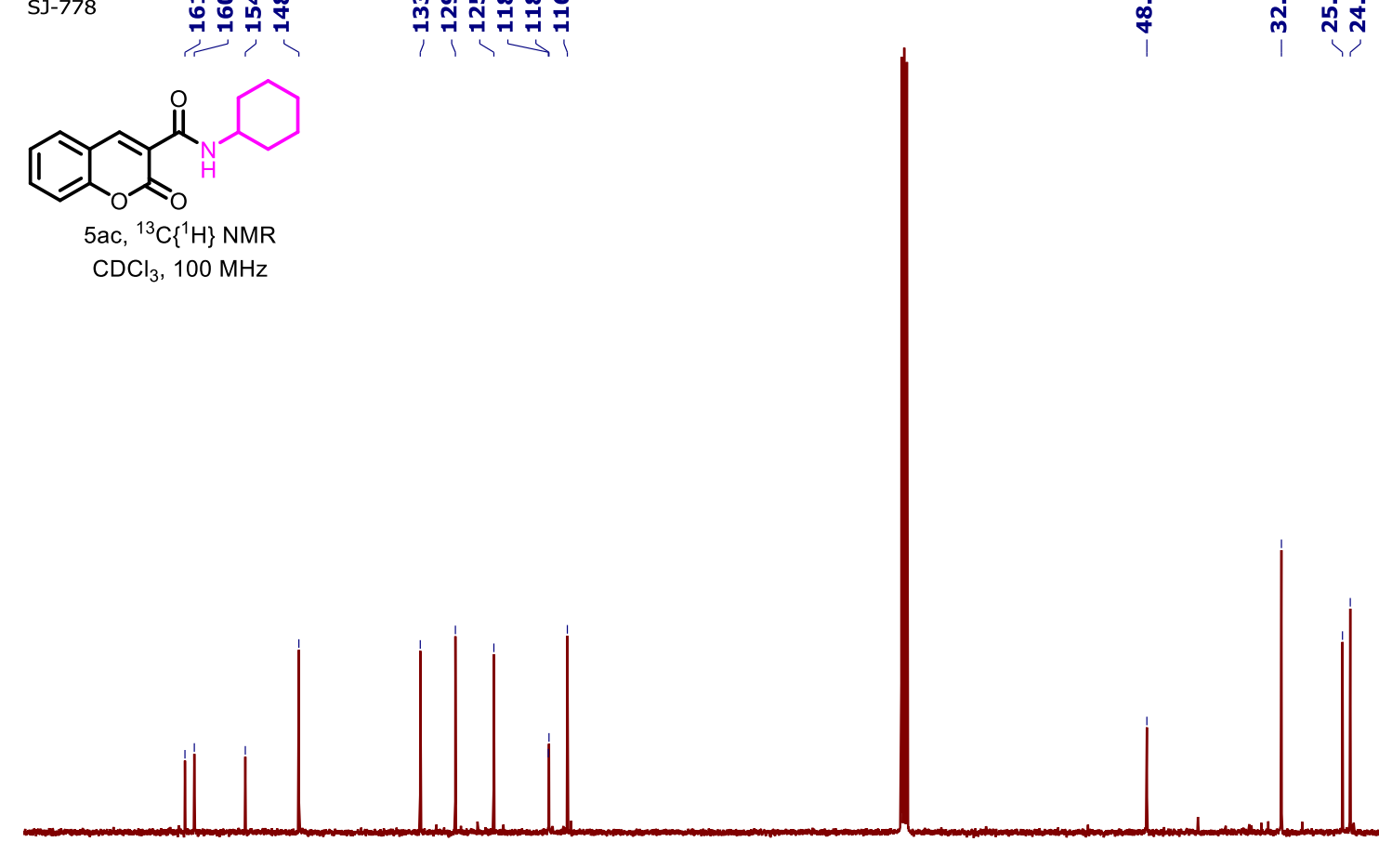

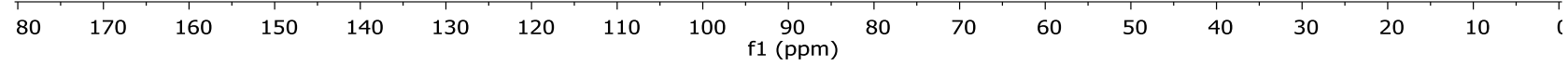


<smiles>O=C(NCc1ccccc1)c1cc2ccccc2oc1=O</smiles>

5ad, ${ }^{1} \mathrm{H}$ NMR

$\mathrm{CDCl}_{3}, 400 \mathrm{MHz}$

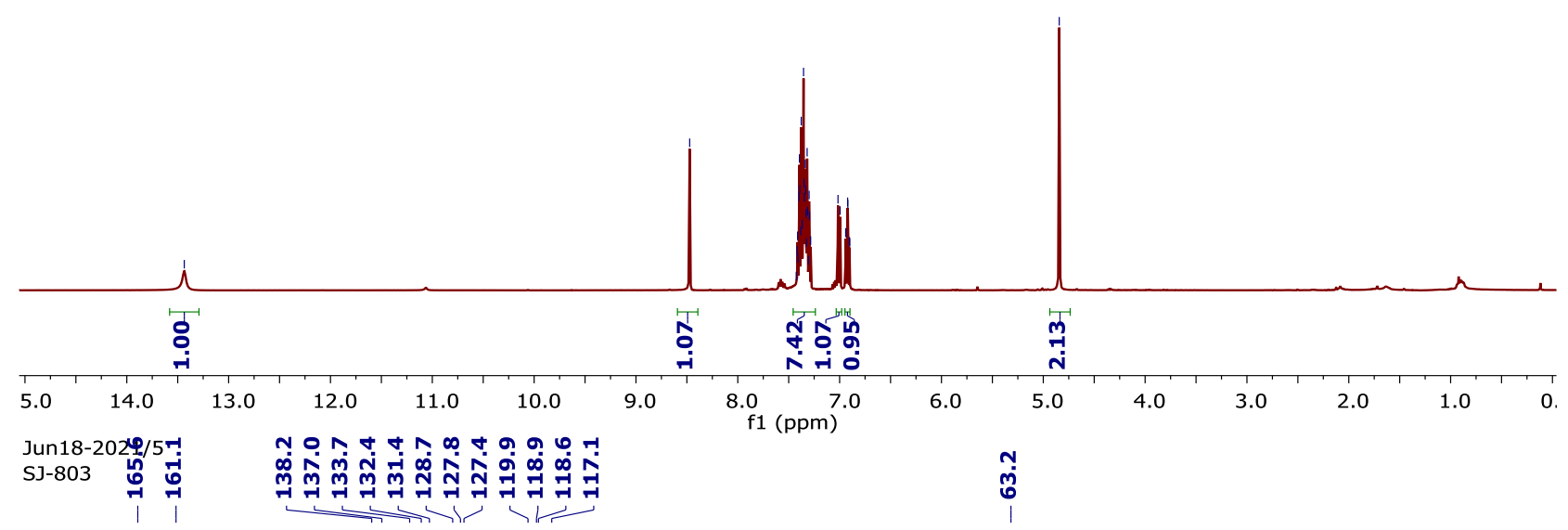<smiles>O=C(NCc1ccccc1)c1cc2ccccc2oc1=O</smiles>

5ad, ${ }^{13} \mathrm{C}\left\{{ }^{1} \mathrm{H}\right\}$ NMR

$\mathrm{CDCl}_{3}, 100 \mathrm{MHz}$

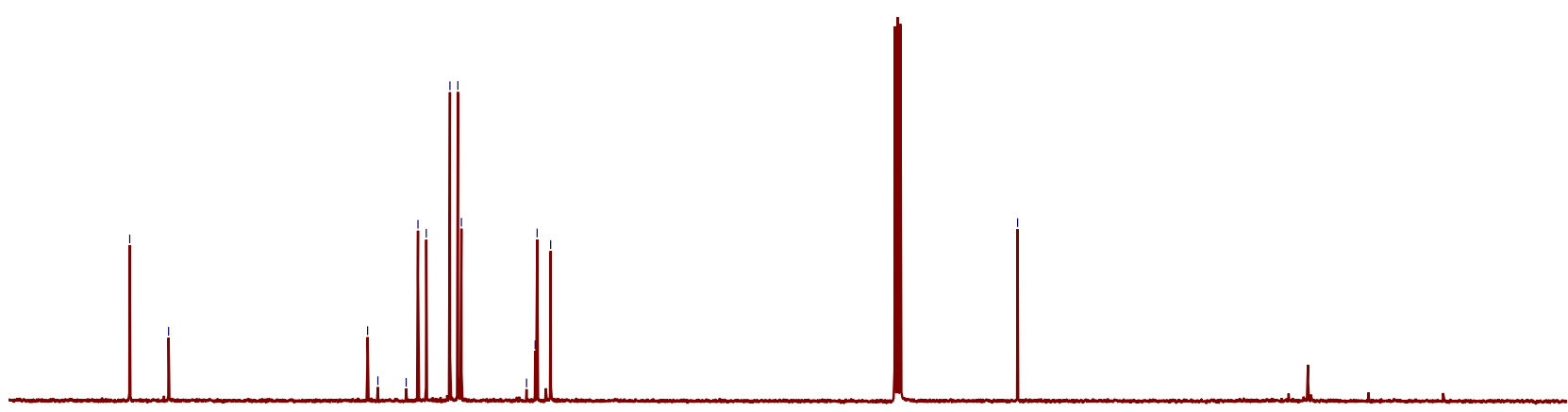

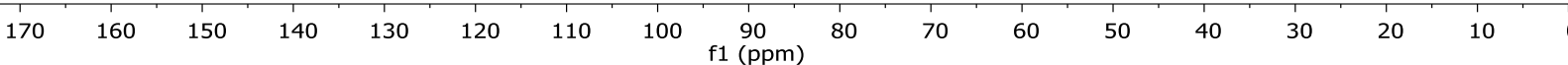




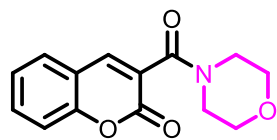

5ae, ${ }^{1} \mathrm{H}$ NMR

$\mathrm{CDCl}_{3}, 400 \mathrm{MHz}$

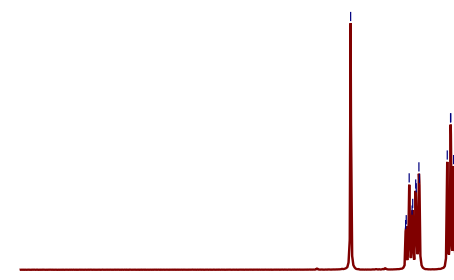

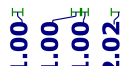

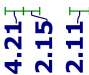

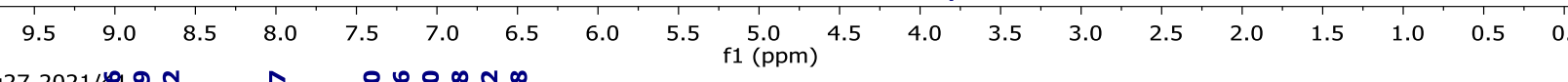

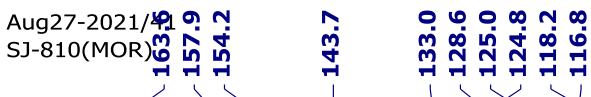

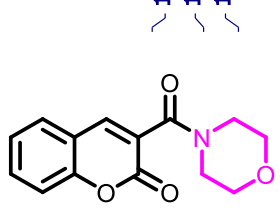

5ae, ${ }^{13} \mathrm{C}\left\{{ }^{1} \mathrm{H}\right\}$ NMR

$\mathrm{CDCl}_{3}, 100 \mathrm{MHz}$

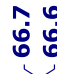

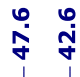

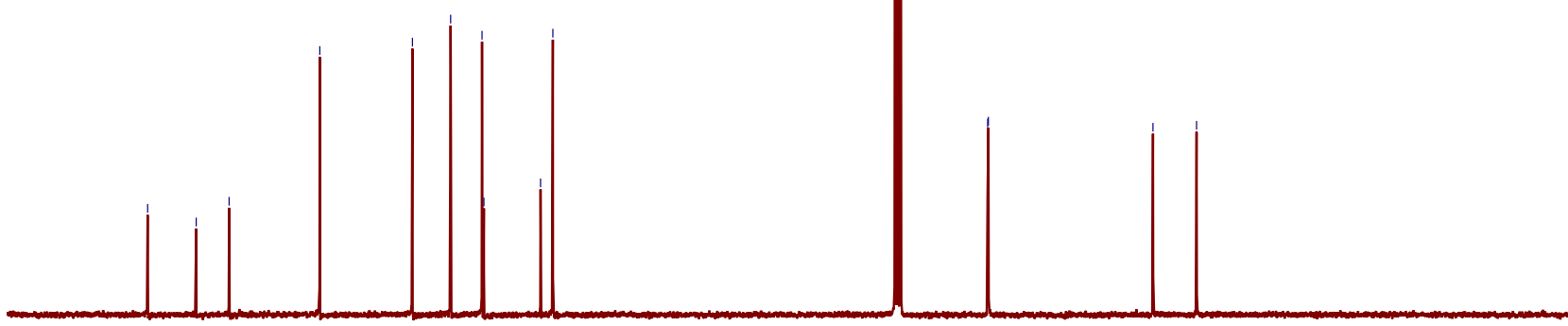

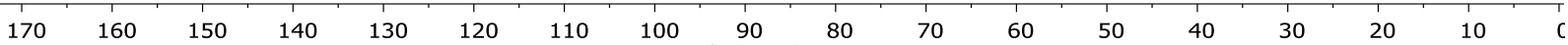



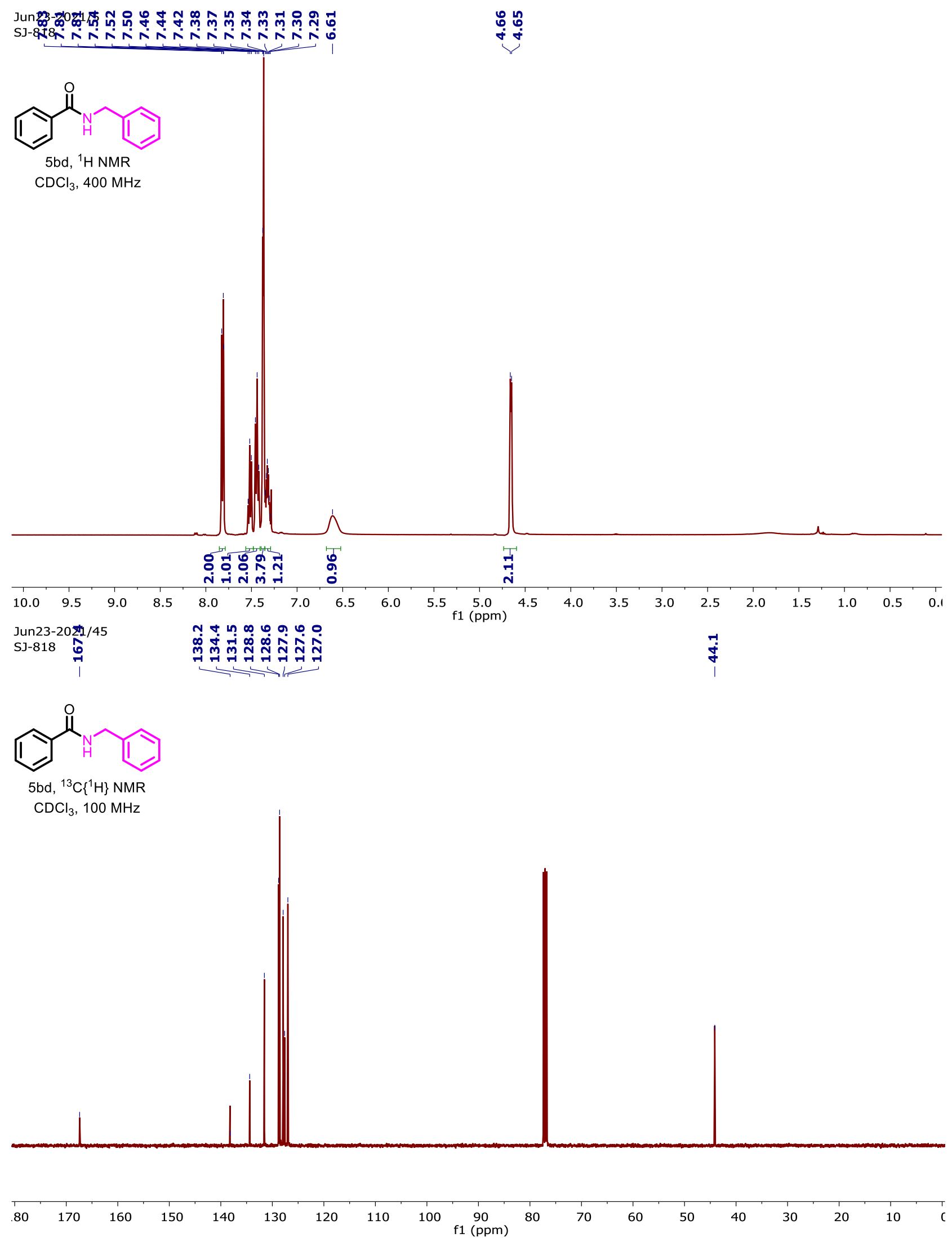


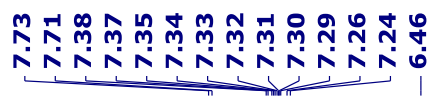

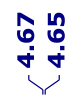

$\underset{\text { กิ }}{\text { กิ }}$
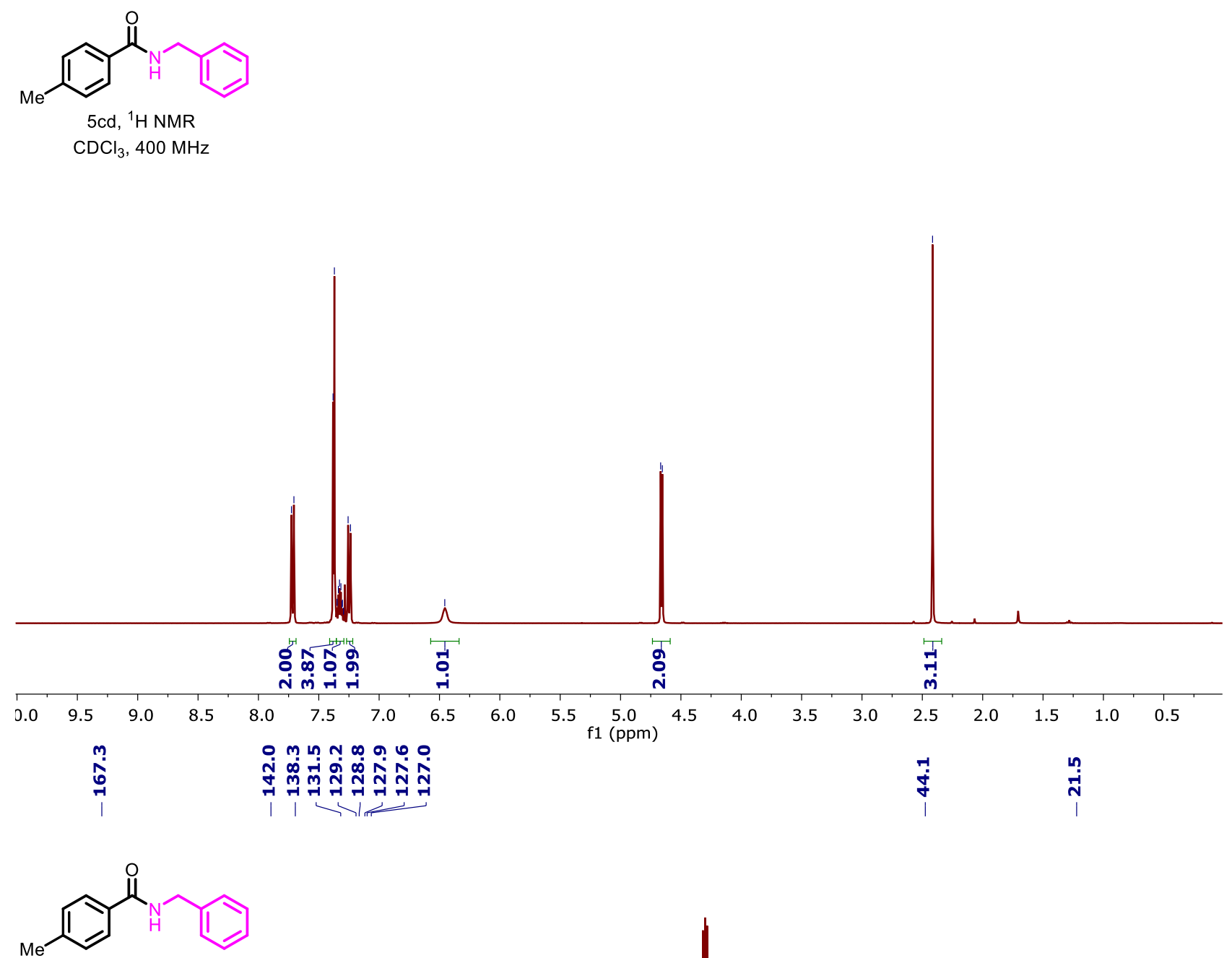

$5 \mathrm{~cd},{ }^{13} \mathrm{C}\left\{{ }^{1} \mathrm{H}\right\}$ NMR $\mathrm{CDCl}_{3}, 100 \mathrm{MHz}$
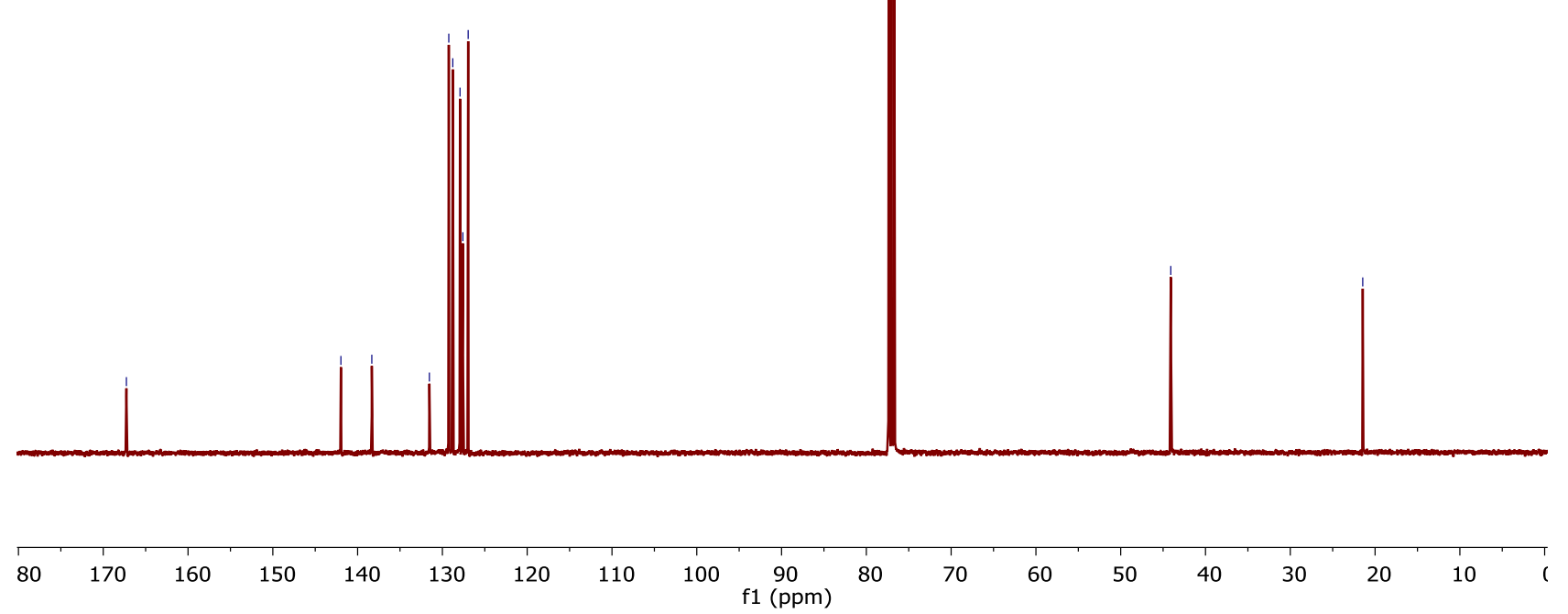

S41 


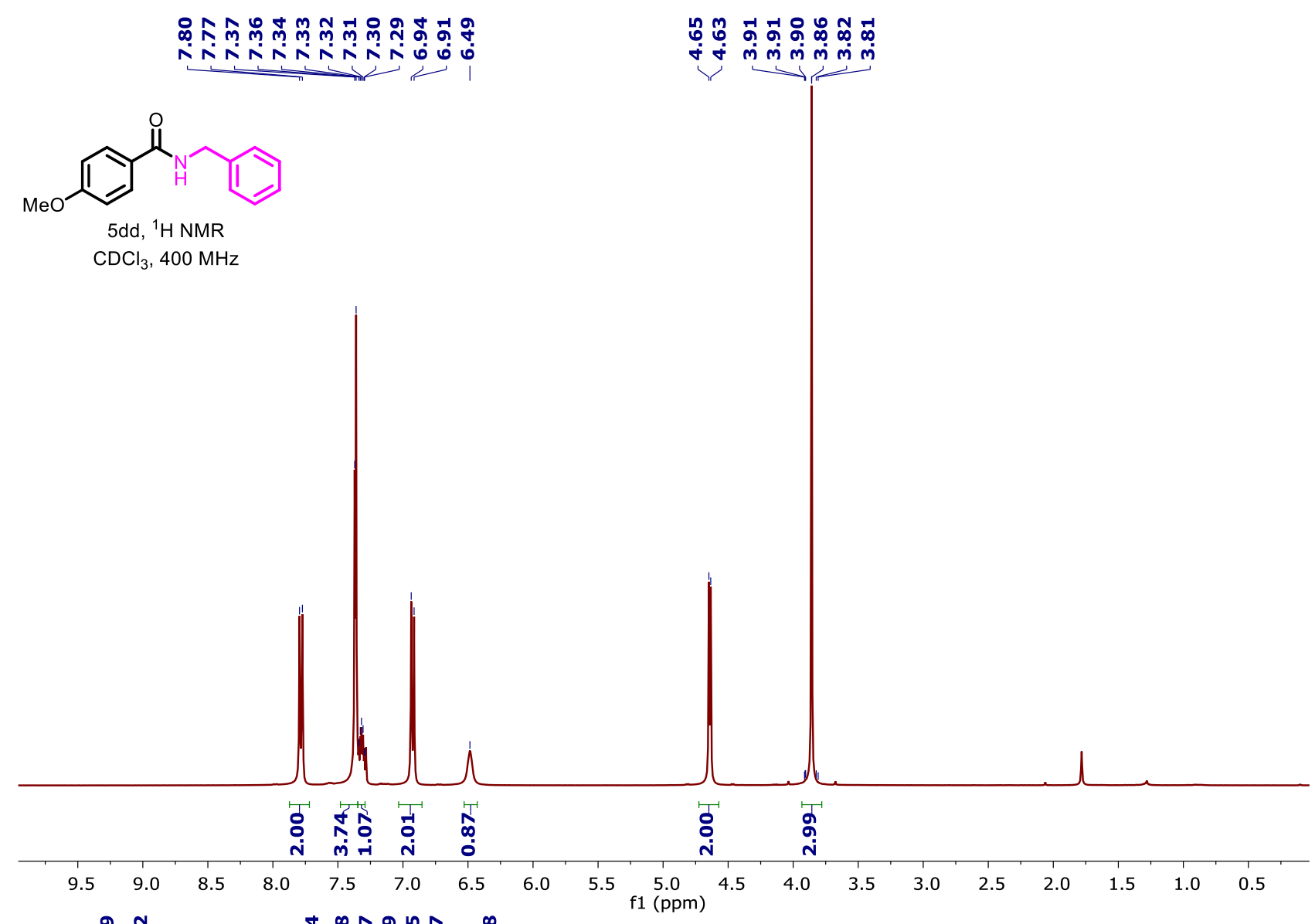

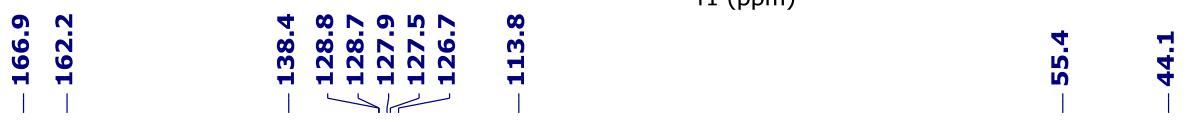

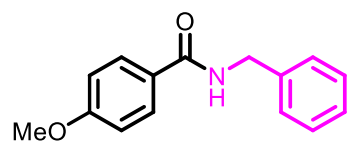

$5 \mathrm{dd},{ }^{13} \mathrm{C}\left\{{ }^{1} \mathrm{H}\right\}$ NMR

$\mathrm{CDCl}_{3}, 100 \mathrm{MHz}$

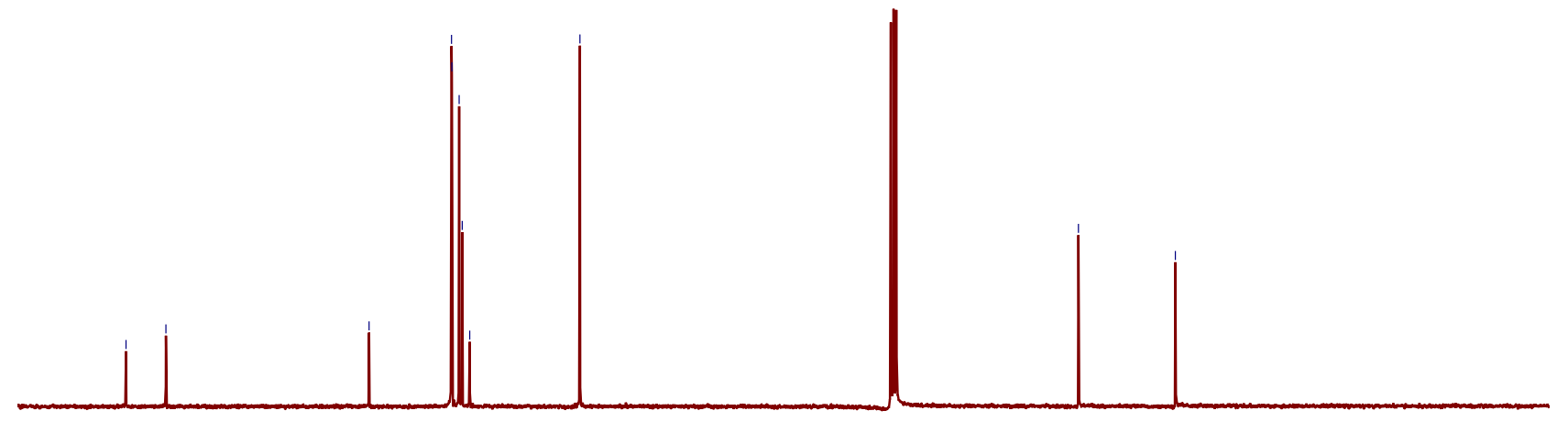

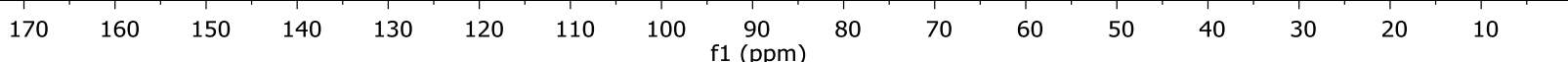




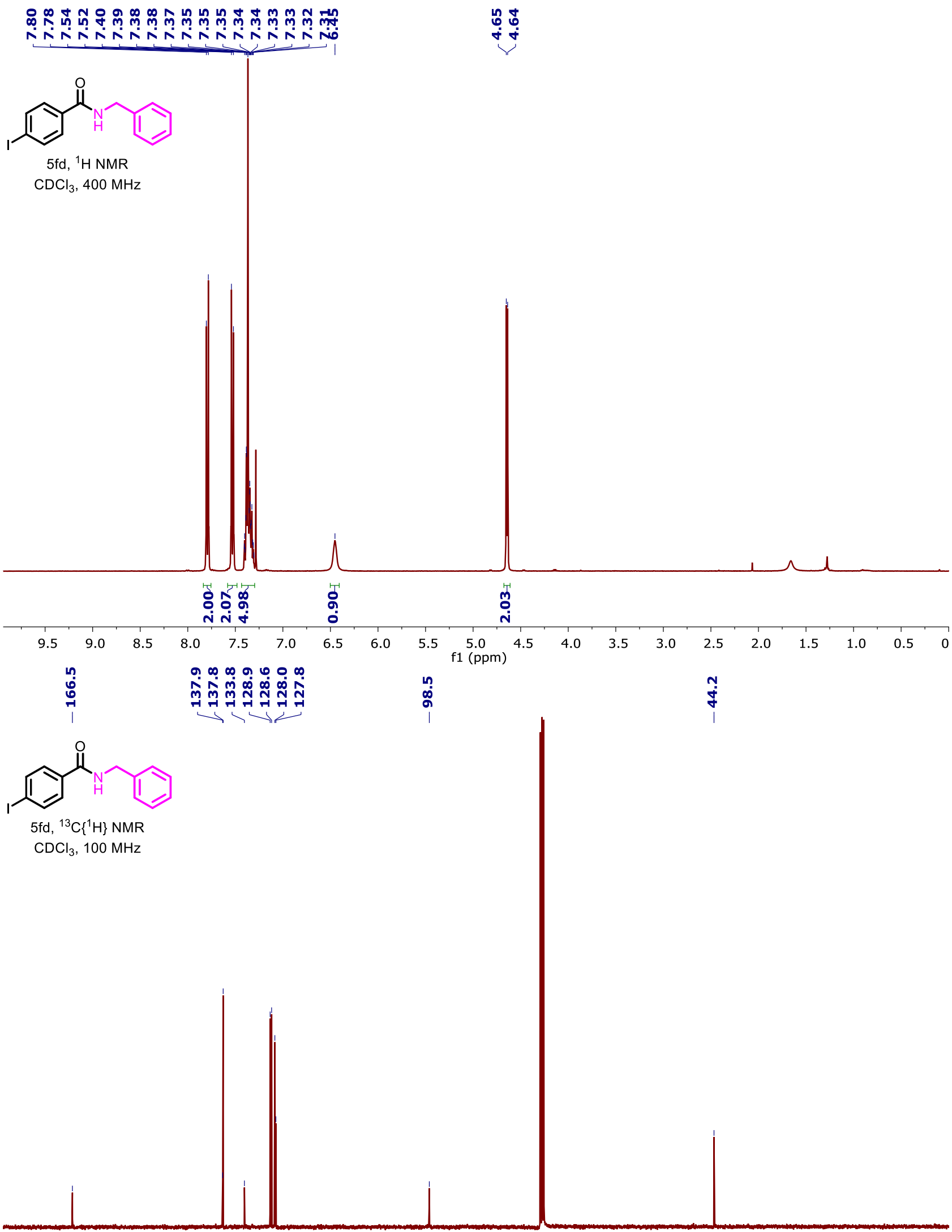

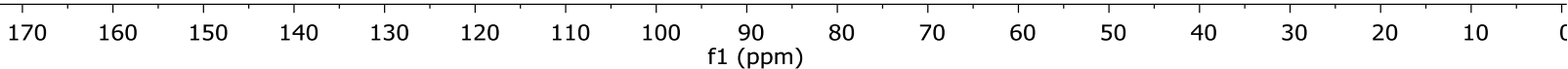


<smiles>O=C(NCc1ccccc1)c1ccc([N+](=O)[O-])cc1</smiles>

$5 \mathrm{gd},{ }^{1} \mathrm{H}$ NMR

$\mathrm{CDCl}_{3}, 400 \mathrm{MHz}$
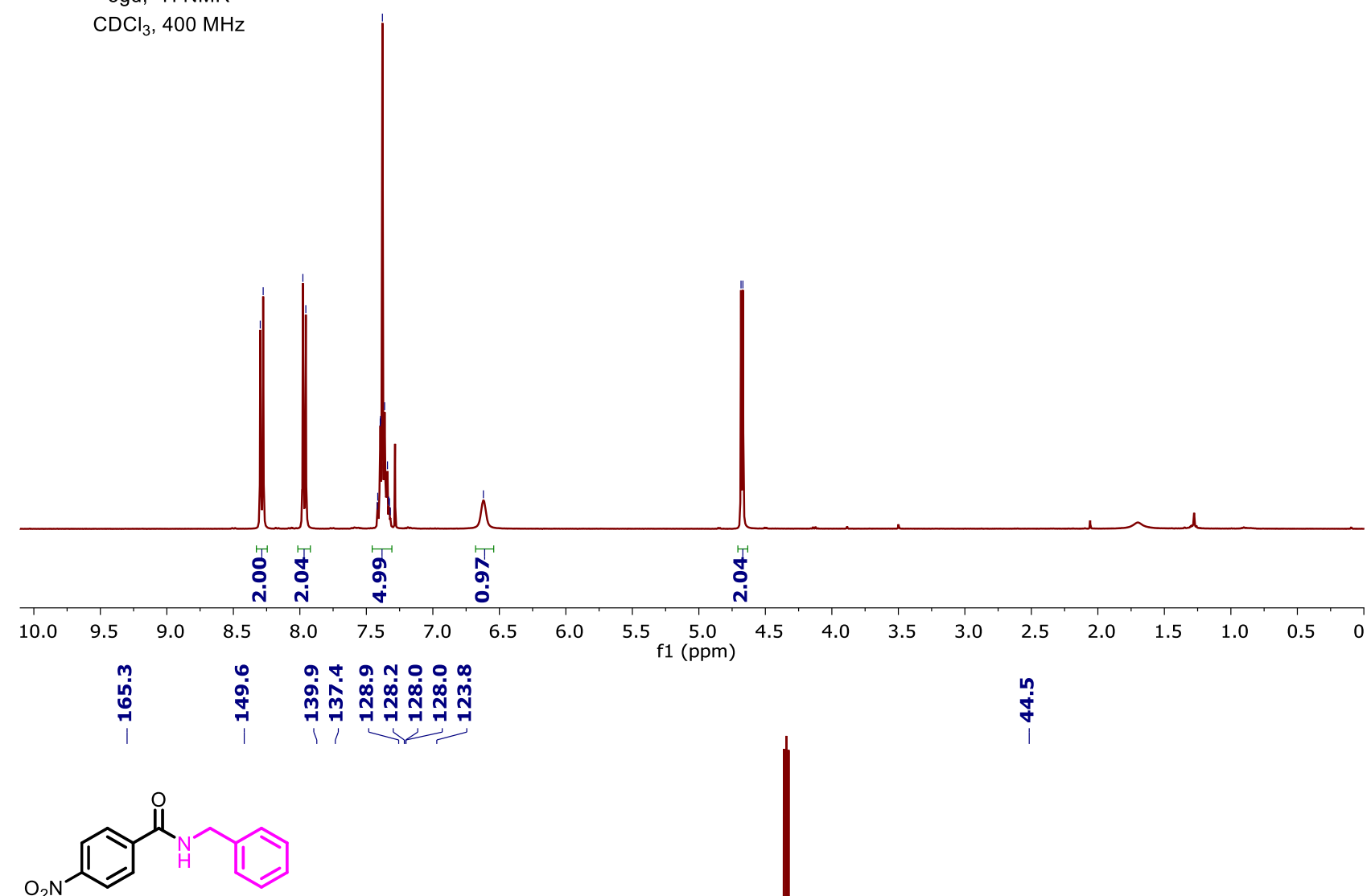

$5 \mathrm{gd},{ }^{13} \mathrm{C}\left\{{ }^{1} \mathrm{H}\right\}$ NMR

$\mathrm{CDCl}_{3}, 100 \mathrm{MHz}$

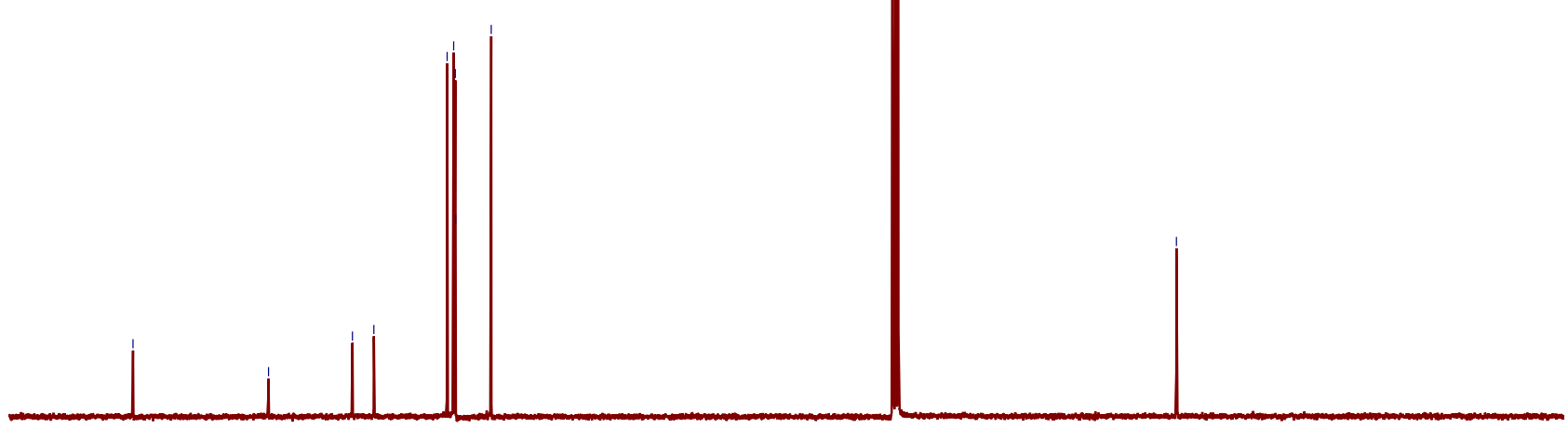

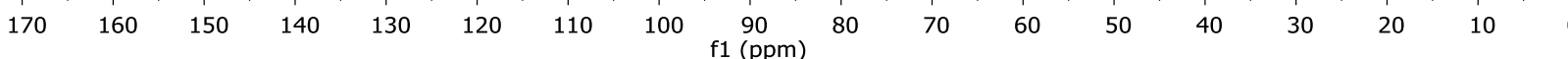




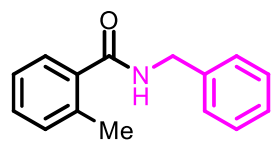

5hd, ${ }^{1} \mathrm{H}$ NMR

$\mathrm{CDCl}_{3}, 400 \mathrm{MHz}$
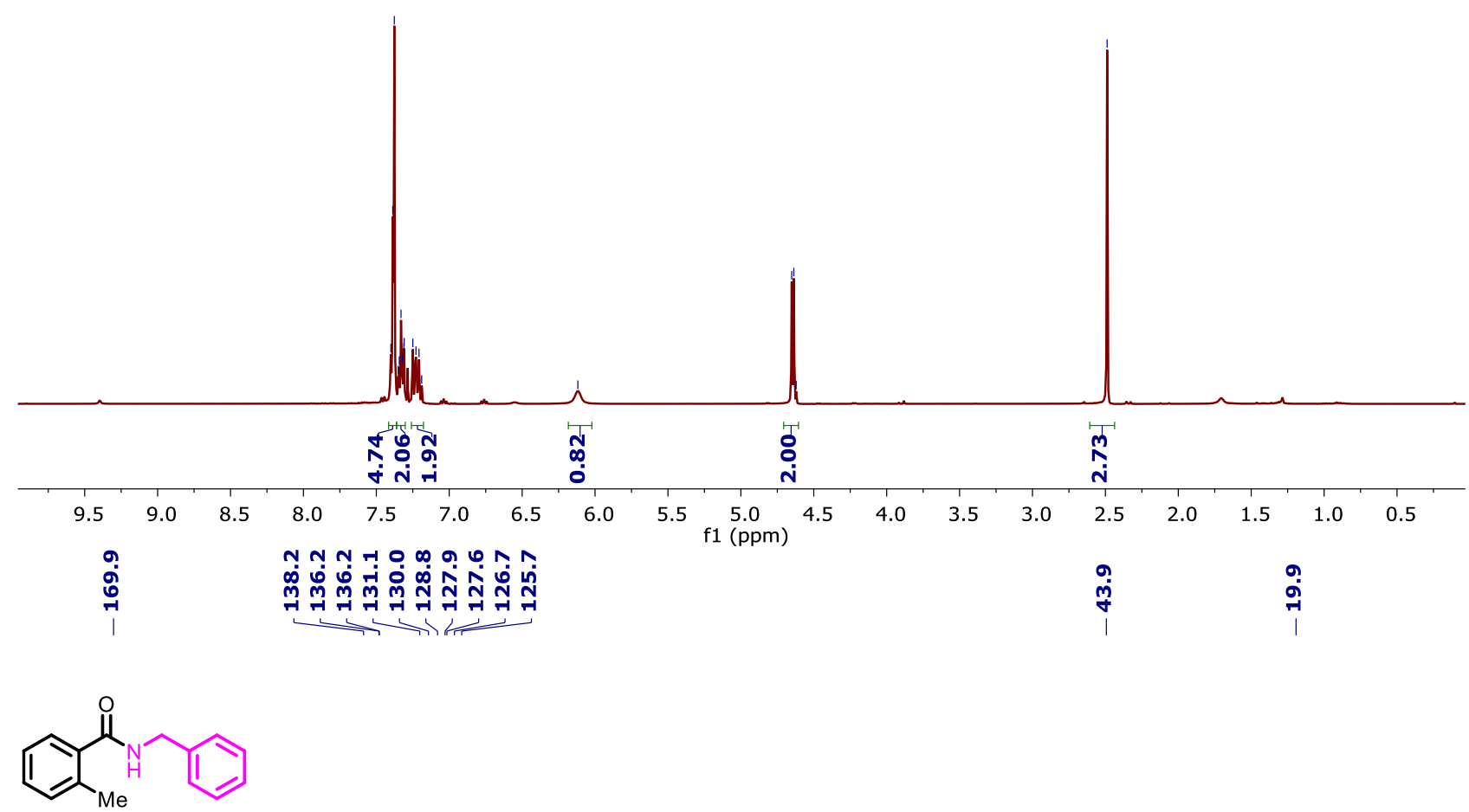

5hd, ${ }^{13} \mathrm{C}\left\{{ }^{1} \mathrm{H}\right\}$ NMR

$\mathrm{CDCl}_{3}, 100 \mathrm{MHz}$

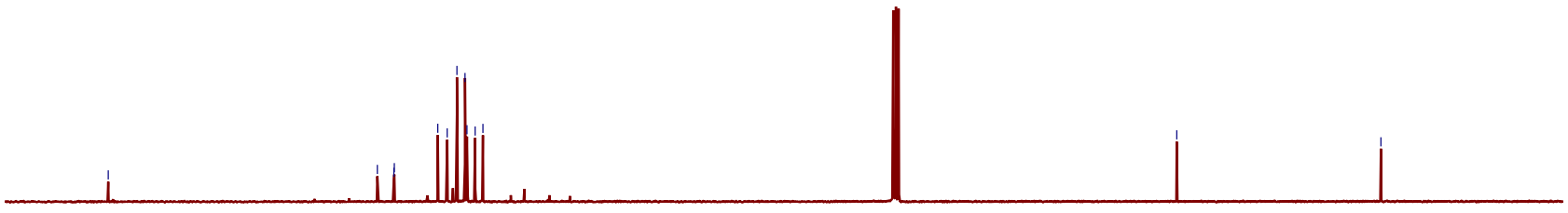

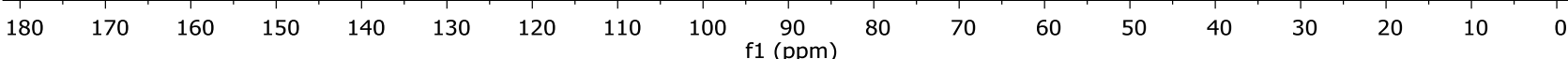




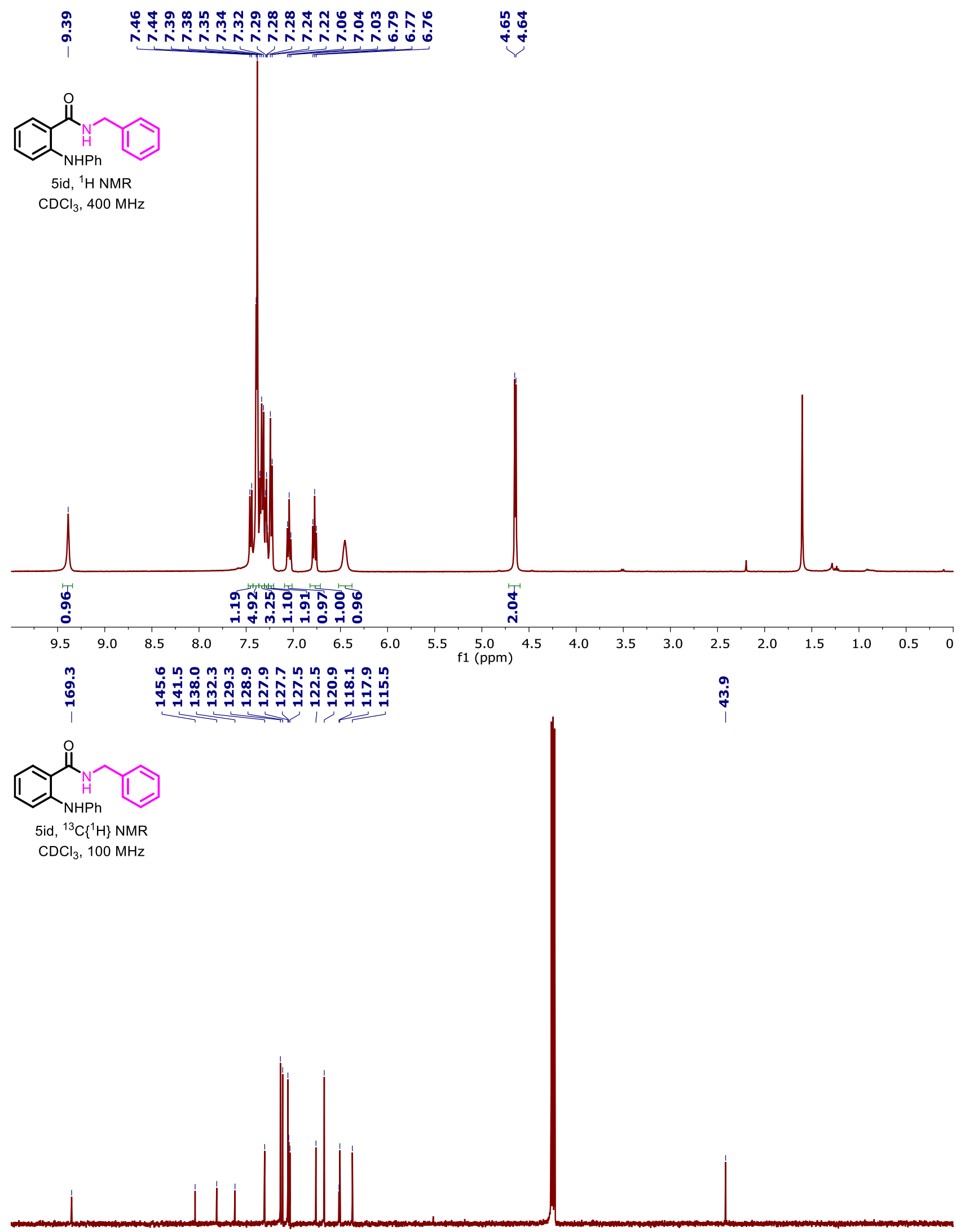

180

$\begin{array}{lllll}170 & 160 & 150 & 140 & 130\end{array}$

120

110

100

90
$1(\mathrm{ppm})$

$70 \quad 60$

50

$40 \quad 30$

$20 \quad 10$

10 
<smiles>O=C(NCc1ccccc1)c1ccccn1</smiles>

5ld, ${ }^{1} \mathrm{H}$ NMR

$\mathrm{CDCl}_{3}, 400 \mathrm{MHz}$
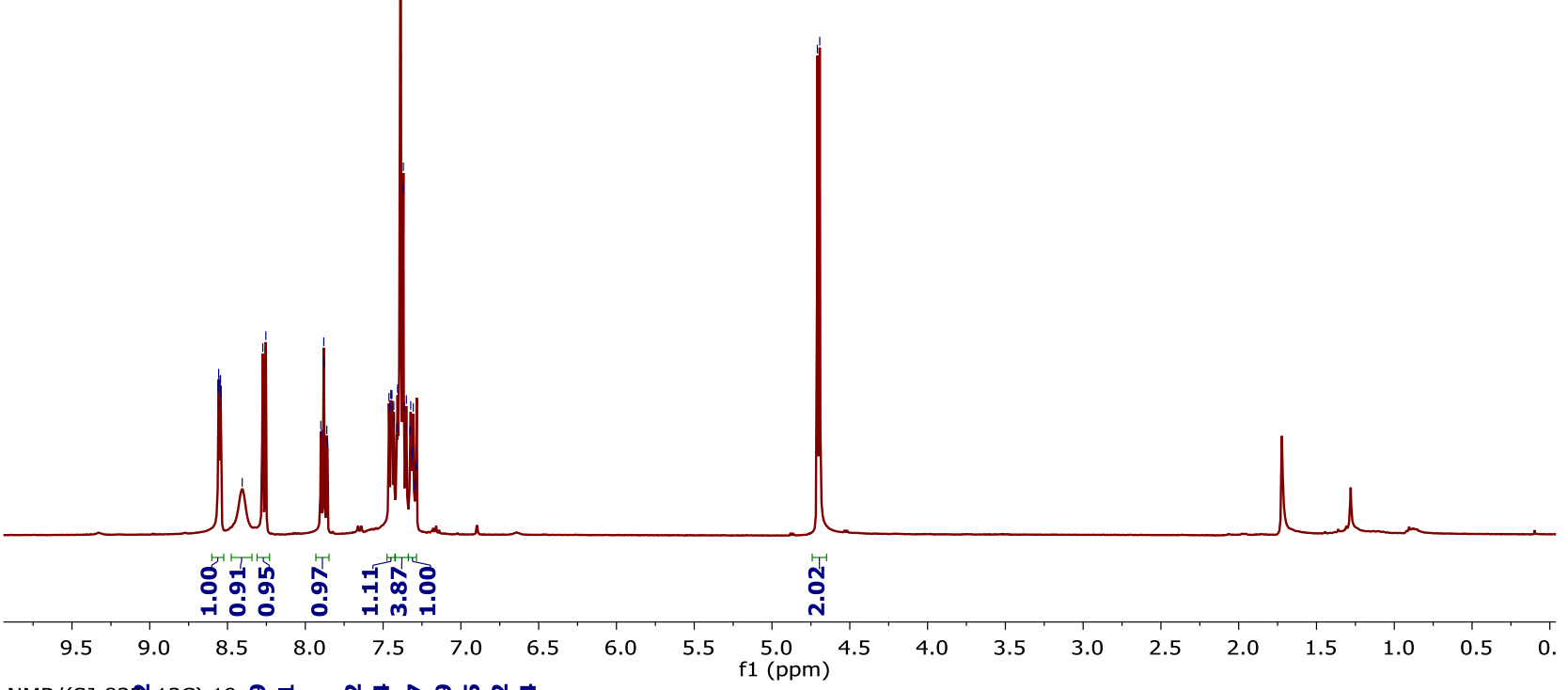

NMR/(SJ-832113C) 19 Oे

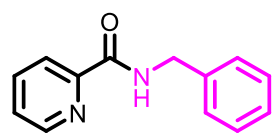

$5 \mathrm{dd},{ }^{13} \mathrm{C}\left\{{ }^{1} \mathrm{H}\right\} \mathrm{NMR}$

$\mathrm{CDCl}_{3}, 100 \mathrm{MHz}$

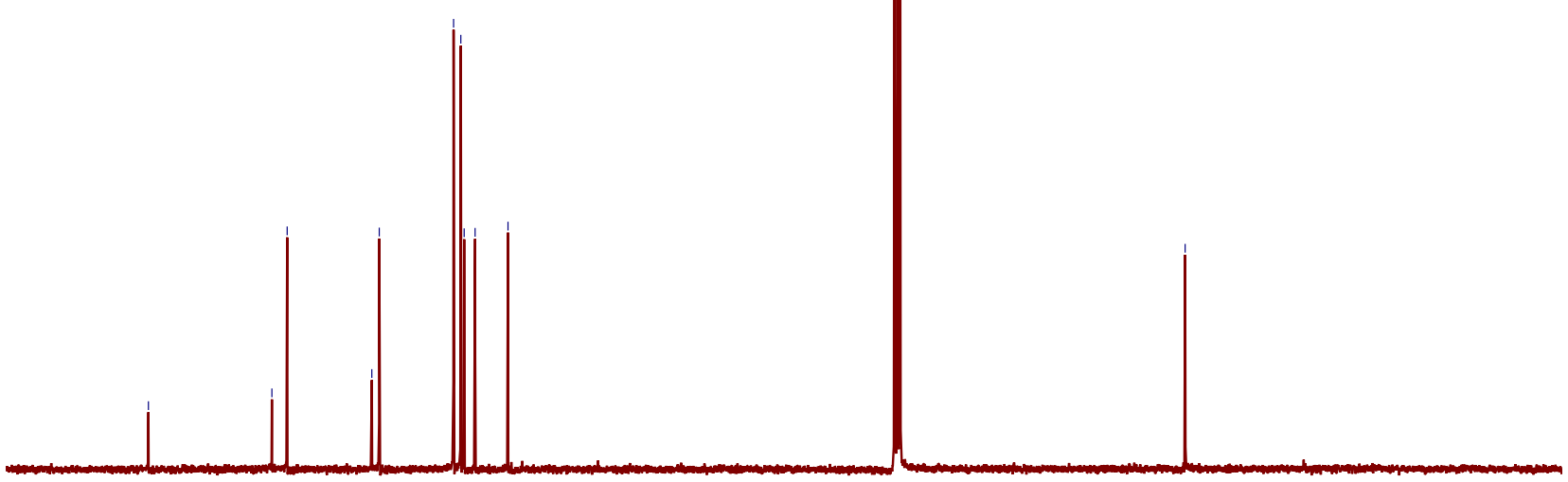

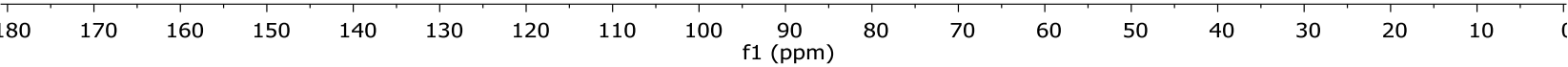


ลำ

诲

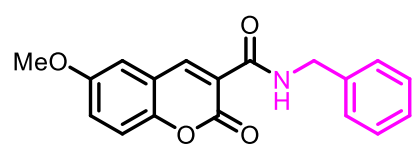

5nd, ${ }^{1} \mathrm{H}$ NMR

$\mathrm{CDCl}_{3}, 400 \mathrm{MHz}$
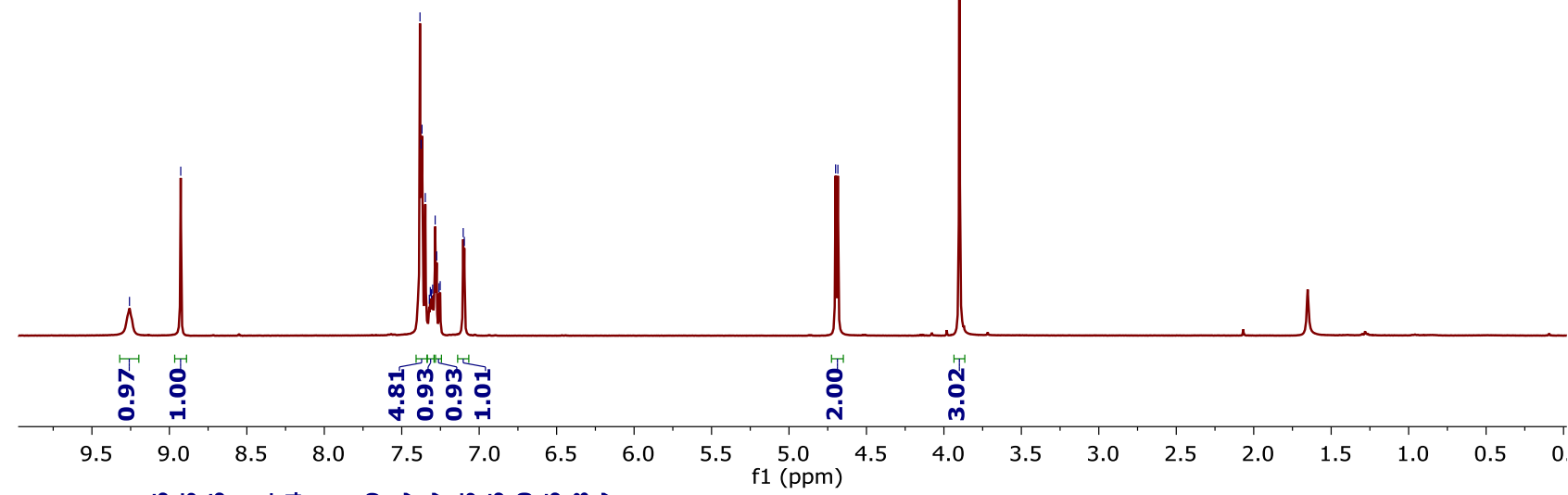

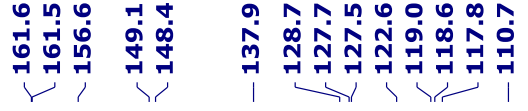

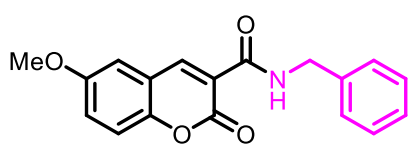

5nd, ${ }^{13} \mathrm{C}\left\{{ }^{1} \mathrm{H}\right\}$ NMR

$\mathrm{CDCl}_{3}, 100 \mathrm{MHz}$

㝒

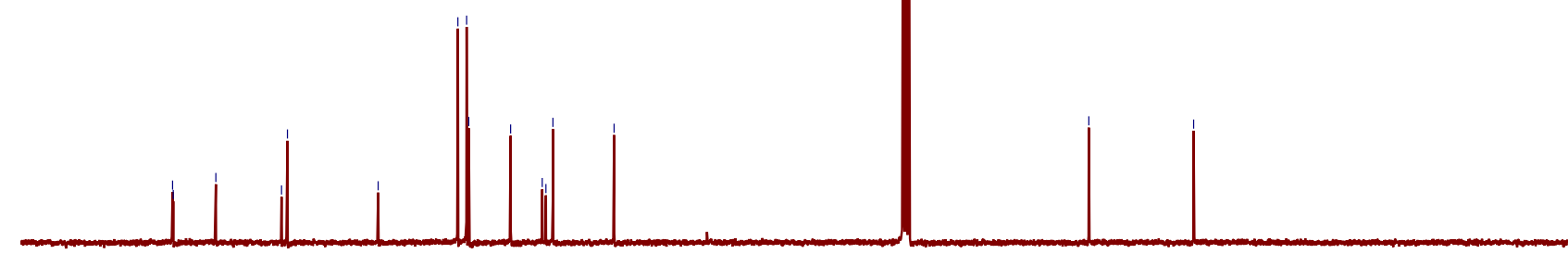




\section{Copy of HMRS of $1 t^{-18} \mathrm{O}$ and intermediate}
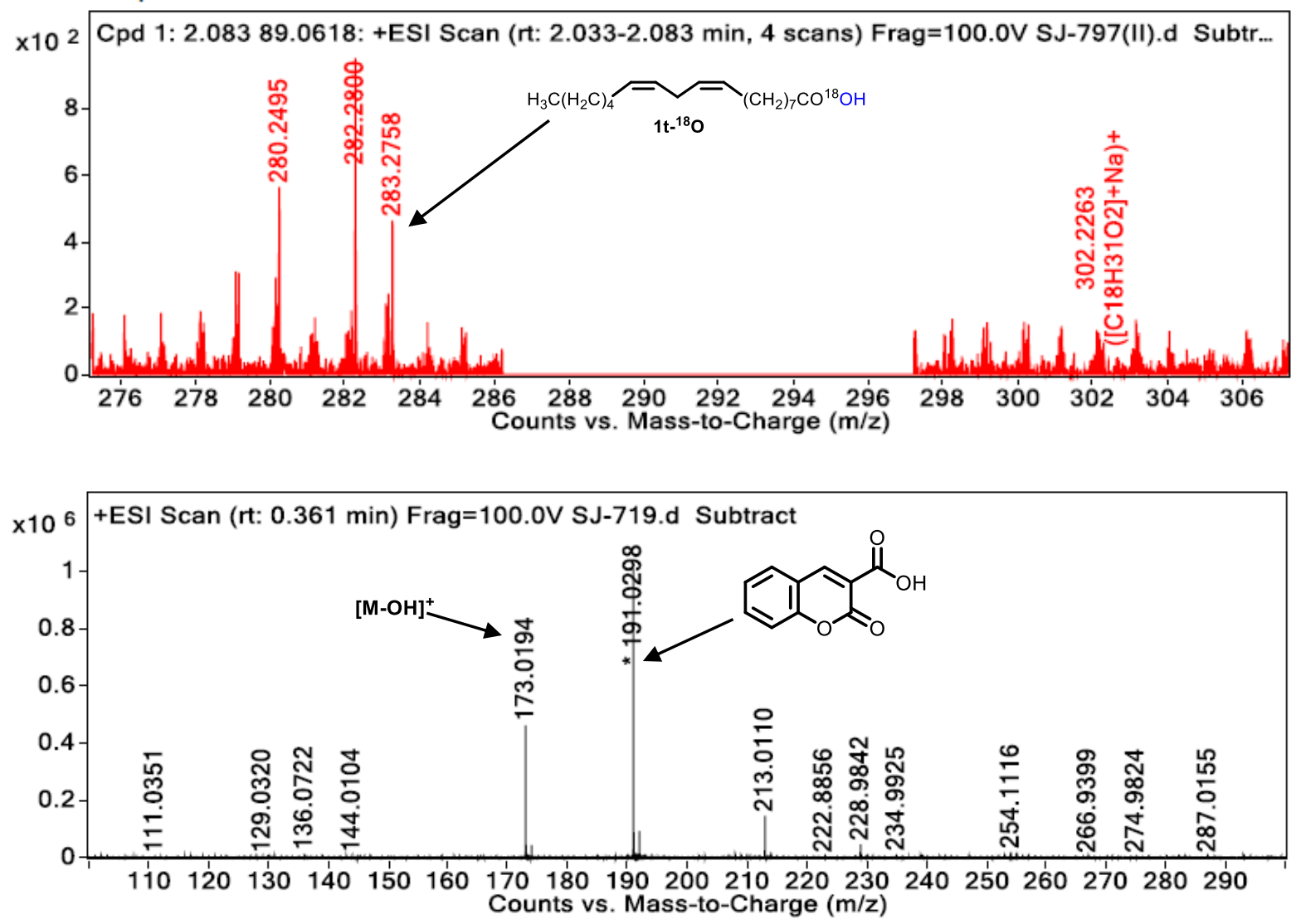

\section{Calculations for green matrics ${ }^{1}$}

\section{a) For this method}

Table S1: Calculation of green chemistry metrics

\begin{tabular}{|c|c|c|c|c|c|}
\hline Steps & Reagent 1 & Reagent 2 & Solvent & Product & Yield \\
\hline \multirow{3}{*}{1} & Levulinic acid (1u) & Ethanol $(\mathbf{2 b})$ & Ethanol & Ethyl levulinate & \\
& MW: $116.11 \mathrm{~g} / \mathrm{mol}$ & MW: $46.07 \mathrm{~g} / \mathrm{mol}$ & $\mathrm{MW}: 46.07 \mathrm{~g} / \mathrm{mol}$ & $(\mathbf{3 u b}), 1.142 \mathrm{~g}$ & $92 \%$ \\
& Amount: $1.0 \mathrm{~g}$ & Amount: $0.4 \mathrm{~g}(0.5 \mathrm{~mL})$ & Amount: $2.0 \mathrm{~g}(2.5 \mathrm{~mL})$ & \\
\hline
\end{tabular}

$\%$ Yield $=\frac{\text { Mass of desired product }}{\text { Theoretical yield of product }} \times 100 \%=\frac{1.142}{1.241} \times 100 \%=92 \%$

Atom economy $=\frac{\text { Molecular mass of desired product }}{\text { Molecular mass of all reactants }} \times 100 \%$

$$
=\frac{144.2}{116.1+46.1} \times 100 \%=88.8 \%
$$

Atom efficiency $=\quad \%$ Yield $\times$ Atom economy $=92 \times 0.89=81.7$

$\mathrm{E}-$ factor $=\frac{\text { Mass of all reactants and solvent }- \text { mass of product }}{\text { Mass of desired product }}$

With solvent recycling

$$
=\frac{1000+394-1142}{1142}=0.22
$$


Without solvent recycling

$$
\begin{aligned}
& =\frac{1000+2394-1142}{1142}=1.97 \\
& \text { Reaction mass efficiency }=\frac{\text { Mass of desired product }}{\text { Mass of reactants used }} \times 100 \\
& =\frac{1.142}{1.000+0.400}=0.816
\end{aligned}
$$

b) For lipase catalyzed method ${ }^{2}$

\begin{tabular}{|c|c|c|c|c|c|c|}
\hline Steps & Reagent 1 & Reagent 2 & Catalyst & Solvent & Product & Yield \\
\hline \multirow{2}{*}{1} & Levulinic acid & Ethanol & $25 \mathrm{mg}$ & MTBE & Ethyl levulinate & $95.9 \%$ \\
& $116.1 \mathrm{mg}$ & $460.7 \mathrm{mg}$ & & $740 \mathrm{mg}(1 \mathrm{~mL})$ & $138.27 \mathrm{~g}$ & \\
\hline
\end{tabular}

Atom economy $=88.8 \%$

Atom efficiency $=88.8 \times 0.959=85.16$

E - factor (With solvent recycling)

$$
=\frac{116.1+460.7+25-138.27}{138.27}=3.35
$$

E - factor (Without solvent recycling)

$$
=\frac{116.1+460.7+25+740-138.27}{138.27}=8.70
$$

\section{References}

1. (a) Clark, J. H.; Farmer, T. J.; Macquarrie, D. J.; Sherwood, J. Using Metrics and Sustainability Considerations to Evaluate the Use of Bio-Based and Non-Renewable Brønsted Acidic Ionic Liquids to Catalyse Fischer Esterification Reactions. Sustain. Chem. Process. 2013, 1, 1-13; (b) Constable, D. J.; Curzons, A. D.; Cunningham, V. L. Metrics to 'Green' Chemistry-Which are the Best? Green Chem. 2002, 4, 521-527; (c) Sheldon, R. A. The E Factor 25 Years On: The Rise of Green Chemistry and Sustainability. Green Chem. 2017, 19, 18-43.

2. Jia, B.; Liu, C.; Qi, X. Selective Production of Ethyl Levulinate from Levulinic Acid by LipaseImmobilized Mesoporous Silica Nanoflowers Composite. Fuel Process. Technol. 2020, 210, 106578. 